\title{
Organocatalyzed Fluoride Metathesis
}

\author{
Daniel Mulryan, Andrew J. P. White and Mark R. Crimmin*
}

Department of Chemistry, Molecular Sciences Research Hub, Imperial College London, White City, Shepherds Bush, W12 OBZ, UK 


\section{Contents:}

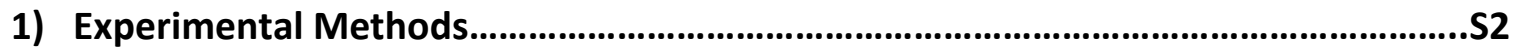

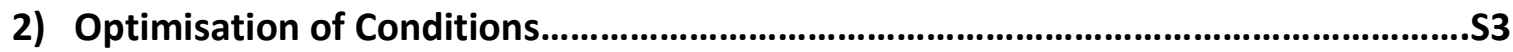

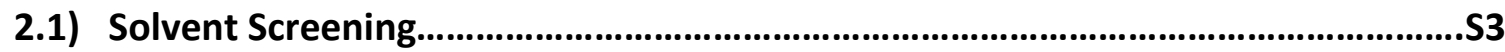

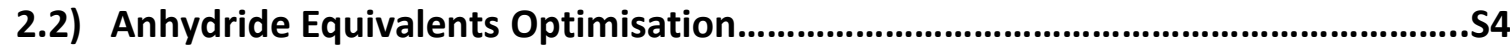

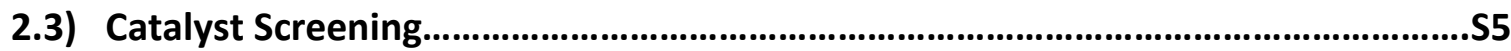

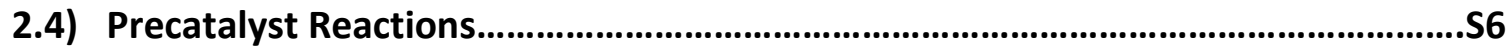

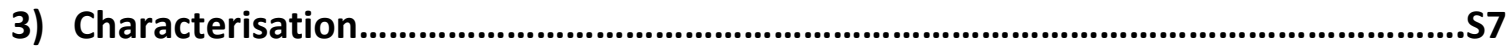

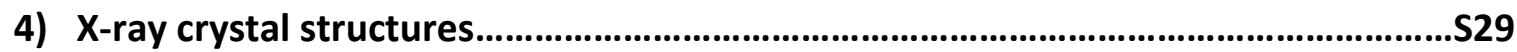

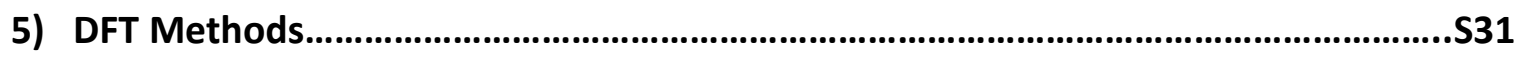

5.1) Dual Catalysed Pathway................................................................................................S31

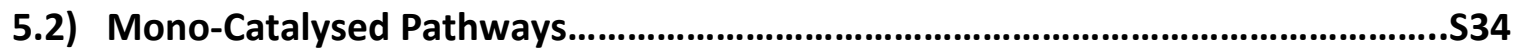

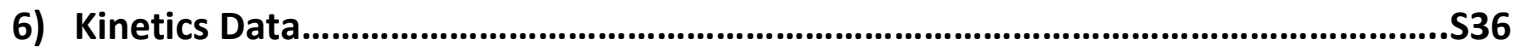

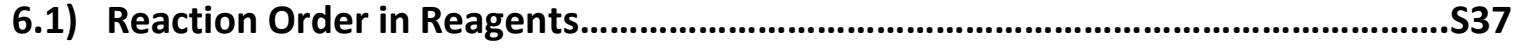

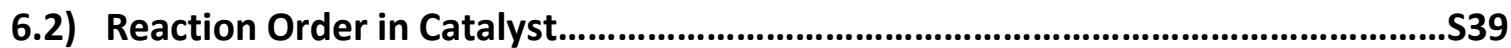

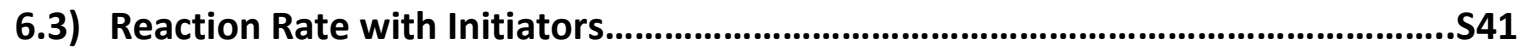

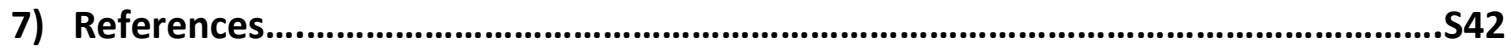

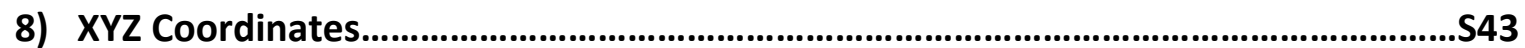

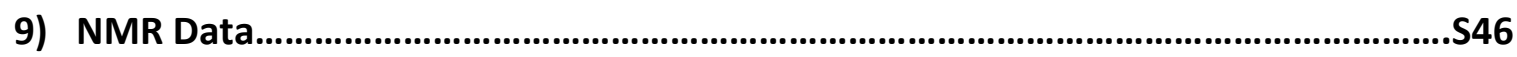




\section{Experimental Methods.}

Reactions were carried out using standard Schlenk-line and glovebox techniques. All reagents were manipulated in a glovebox, with the exception of acetonitrile, which was added to reaction mixtures in a fumehood. Reactions were carried out in J. Young's tap NMR tubes. Glassware was dried for 12 hours at $120^{\circ} \mathrm{C}$ prior to use. All solvents were dried over activated alumina from an SPS (solvent purification system) and subsequently degassed, prior to use. Benzene- $d_{6}$ was stored over $3 \AA$ molecular sieves. Reactions were heated using silicone oil baths. ${ }^{1} \mathrm{H},{ }^{13} \mathrm{C}$ and ${ }^{19} \mathrm{~F}$ NMR spectra were obtained on BRUKER $400 \mathrm{MHz}$ or $500 \mathrm{MHz}$ machines and referenced against $\mathrm{SiMe}_{4}\left({ }^{1} \mathrm{H},{ }^{13} \mathrm{C}\right)$ or $\mathrm{CFCl}_{3}\left({ }^{19} \mathrm{~F}\right)$. All peak intensities are derived against an internal standard peak of 1,4-difluorobenzene $\left(\delta_{\mathrm{F}}=-121.1 \mathrm{ppm}\right)$. A 55s delay was used for quantitative ${ }^{19} \mathrm{~F}$ NMR integration. NMR data was processed using MestReNova software. Multiplicity assignments in NMR spectra are labelled as follows: " $\mathrm{s}$ " = singlet, " $\mathrm{d}$ "= doublet, " $\mathrm{t}$ "= triplet, " $\mathrm{m}$ " = multiplet.

Reagents were purchased from Sigma Aldrich. All reagents were stored under an inert atmosphere, with liquid reagents being dried over activated $3 \AA$ molecular sieves and freezepump-thaw degassed before use. Purifications were carried out by column chromatography on silica gel (tech grades, $60 \AA$, 230-400 mesh, 40-63 $\mu \mathrm{m}$ particle size) using a ratio of 9:1 parts n-pentane : DCM, or a ratio of 19:1 parts n-hexane: ethyl acetate. 


\subsection{Solvent Screening:}<smiles>Fc1nc(F)c(F)c(F)c1F</smiles><smiles>C[PH3+][PH2+]C(=O)OC(=O)c1ccccc1</smiles>

DMAP (0.05 equiv)

$17 \mathrm{hrs}$<smiles>O=C(Oc1c(F)c(F)nc(F)c1F)c1ccccc1</smiles>

A<smiles>[13CH3]C(=O)[PH2+]</smiles>

B

[0.14 mmol]

[0.14 $\mathrm{mmol}]$

Product A Yield

(\%)

32.6

25.8

100

60

80

100

150
31.4

26.4

35.4

48.3

53.4
Product B Yield (\%)

0

18.9

29.5

57.4

69.7

42.7

Table S1. Reactions performed in J-Youngs NMR tubes with 1:1:0.05 equivalents of pentafluoropyridine (15.4 $\mu$, $0.14 \mathrm{mmol}$ ): benzoic anhydride : DMAP in $0.4 \mathrm{ml}$ of solvent, with a $\mathrm{C}_{6} \mathrm{D}_{6}$ capillary insert. $10 \mu \mathrm{l}$ of $1,4-$ difluorobenzene was added as an internal standard. Yields were calculated using ${ }^{19} \mathrm{~F}$ NMR.

Acetonitrile was found to be the optimal solvent for the reaction. In less polar solvents, poor solubility of the pyridinium fluoride salt intermediate (3a, Figure 2 ) affected reaction rate. In highly polar solvents, little or no benzoyl fluoride formation was observed, owing to stronger solvation of the fluoride anion. Heating to $100^{\circ} \mathrm{C}$ in acetonitrile was found to provide the best reaction rate. Under these conditions, no decomposition of acetonitrile, or side reactivity of acetonitrile with either reagent was observed. 


\subsection{Anhydride Equivalents Optimisation}<smiles>Fc1nc(F)c(F)c(F)c1F</smiles>

[0.14 mmol]<smiles>O=C(OC(=O)c1ccccc1)c1ccccc1</smiles>

Benzoic anhydride equivalents

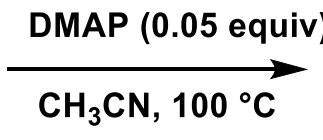

$17 \mathrm{hrs}$<smiles>O=C(Oc1c(F)c(F)nc(F)c1F)c1ccccc1</smiles>

A<smiles>[13CH3]C(=O)[PH2+]</smiles>

B

\begin{tabular}{cccc} 
Entry & $\begin{array}{c}\text { Benzoic anhydride } \\
\text { equivalents }\end{array}$ & Product A Yield (\%) & $\begin{array}{c}\text { Product B Yield } \\
(\%)\end{array}$ \\
\hline 8 & 1.0 & 32.6 & 69.7 \\
9 & 1.2 & 38.7 & 68.2 \\
10 & 1.5 & 61.3 & 78.6 \\
11 & 2.0 & 85.9 & 92.4 \\
12 & 5.0 & 88.3 & 97.6 \\
13 & 10.0 & 95.0 & 95.7
\end{tabular}

Table S2. Reactions performed in J-Youngs NMR tubes with 1:0.05 equivalents of pentafluoropyridine $(15.4 \mu \mathrm{l}$, $0.14 \mathrm{mmol}$ ) : DMAP in $0.4 \mathrm{ml}$ of acetonitrile, with a $\mathrm{C}_{6} \mathrm{D}_{6}$ capillary insert. $10 \mu \mathrm{l}$ of 1,4-difluorobenzene was added as an internal standard. Yields were calculated using ${ }^{19} \mathrm{~F}$ NMR.

Product formation stalled under stoichiometric reagent addition. Significantly higher conversion was observed with an excess of benzoic anhydride, however excess anhydride is not essential for the reaction to occur. No side reactivity of benzoic anhydride was observed, and when scaling up the reaction, excess benzoic anhydride was recovered upon column chromatography. 


\subsection{Organocatalyst Screening}<smiles>Fc1nc(F)c(F)c(F)c1F</smiles>

$0.14 \mathrm{mmol}]$<smiles>[CH2+][PH2+]C(=O)OC(=O)c1ccccc1</smiles>

[1.40 mmol]

\section{Catalyst ( 0.05 equivs) \\ $\mathrm{CH}_{3} \mathrm{CN}, 100{ }^{\circ} \mathrm{C}$}

$17 \mathrm{hrs}$<smiles>O=C(P)Oc1c(F)c(F)nc(F)c1F</smiles>

A<smiles>[13CH3]C(=O)[PH2+]</smiles>

B<smiles>c1ccncc1</smiles><smiles>CN(C)c1ccncc1</smiles><smiles>N#Cc1ccncc1</smiles><smiles>C1CCC2=NCCCC2CC1</smiles><smiles>C1CN=C2CCCN2C1</smiles><smiles>C1CN2CCN1C2</smiles><smiles>N=C(N)[N-][O-]</smiles>

A: $\quad 51.8 \%$

$95.0 \%$

$13.4 \%$

$10.6 \%$

$98.2 \%$ $>99 \%$
$60.9 \%$

$61.2 \%$
$4.1 \%$

$3.4 \%$

Scheme S1. Reactions performed in J-Youngs NMR tubes with 1:10:0.05 equivalents of pentafluoropyridine (15.4 $\mu \mathrm{l}, 0.14 \mathrm{mmol}$ ) : benzoic anhydride : DMAP in $0.4 \mathrm{ml}$ of acetonitrile, with a $\mathrm{C}_{6} \mathrm{D}_{6}$ capillary insert. $10 \mu \mathrm{l}$ of $1,4-$ difluorobenzene was added as an internal standard. Yields were calculated using ${ }^{19} \mathrm{~F}$ NMR spectroscopy. 


\subsection{Precatalyst reactions}

Catalytic amounts (5 mol\%) of pyridinium salt $A$ and $B$ were formed, before pentafluoropyridine $(0.14 \mathrm{mmol})$ and benzoic anhydride $(1.40 \mathrm{mmol})$ were added. After 17 hours at $100{ }^{\circ} \mathrm{C}$, stoichiometric conversion to products was observed in both cases.

S2. A)
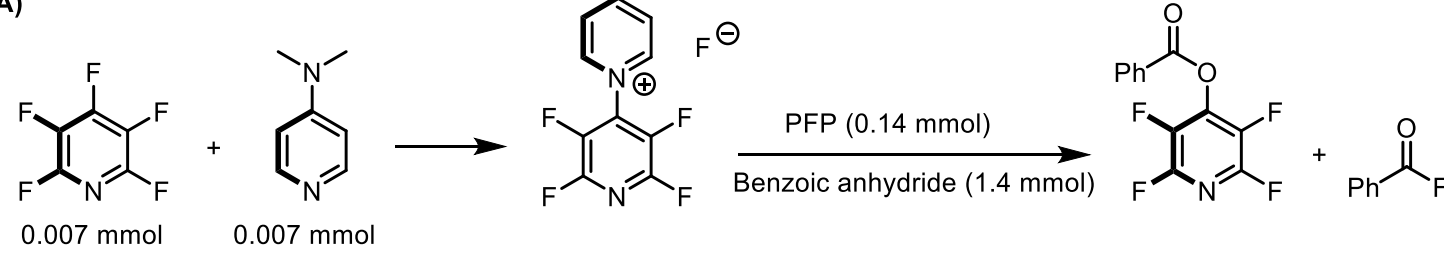

S2. B)

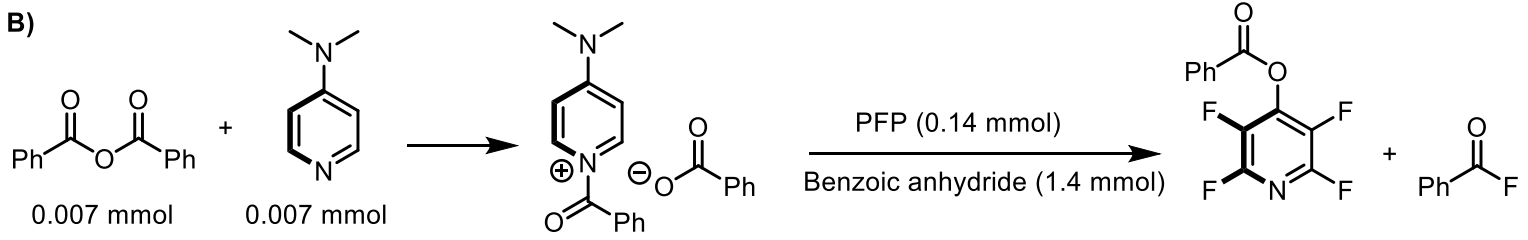

Scheme S2. Reactions performed in J-Youngs NMR tubes, in $0.4 \mathrm{ml}$ of acetonitrile, with a $\mathrm{C}_{6} \mathrm{D}_{6}$ capillary insert. $10 \mu \mathrm{l}$ of 1,4-difluorobenzene was added as an internal standard. Yields were calculated using ${ }^{19} \mathrm{~F}$ NMR.

As reported previously by Sandford and co-workers, the isolation of the pyridinium fluoride salt $A$ was not achieved, owing to its hygroscopic nature. ${ }^{[\mathrm{S} 1]}$ It can, however be observed in solution as peaks at -86.3 and $-146.8 \mathrm{ppm}$ in the ${ }^{19} \mathrm{~F} \mathrm{nmr}$ spectra. 


\subsection{General procedure for fluorine - carbonyl shuttling reactions (1a-i).}

In a glovebox, a fluoroarene (1 equivs, $0.14 \mathrm{mmol}$ ), the carbonyl derivative (10 equiv., 1.40 $\mathrm{mmol})$ and 1,4-difluorobenzene (10 $\mu \mathrm{l}, 0.097 \mathrm{mmol})$ were added to an empty J. Young's NMR tube. The reaction mixture was removed from the glovebox before $0.4 \mathrm{ml}$ of dry acetonitrile was added. ${ }^{a} \mathrm{~A}$ stock solution of 4-N,N-dimethylaminopyridine (DMAP) was made by dissolving $30 \mathrm{mg}$ of DMAP in $4 \mathrm{ml}$ of dry $\mathrm{C}_{6} \mathrm{D}_{6}$. Once a 0 hrs time-point has been taken by ${ }^{19} \mathrm{~F} \mathrm{NMR,} 50 \mu \mathrm{l}$ of this stock solution ( 0.05 equiv., $0.007 \mathrm{mmol}$ ) was added to the reaction mixture. The NMR tube was sealed and heated at $100{ }^{\circ} \mathrm{C}$ for 17 hours in a silicone oil bath. Quantitative ${ }^{19} \mathrm{~F}$ integration was performed using the 1,4-difluorobenzene singlet resonance at $\delta_{\mathrm{F}}-121.1 \mathrm{ppm}$.

Scaled up versions of each reaction were also conducted. In a glovebox pentafluoropyridine (154 $\mu \mathrm{l}, 1$ equiv., $1.4 \mathrm{mmol}$ ), the carbonyl derivative (10 equivs ${ }^{\mathrm{b}}$., $14.0 \mathrm{mmol}$ ), DMAP (0.05 equiv. $0.07 \mathrm{mmol}$ ) and a stirrer bar were added to an empty $25 \mathrm{ml}$ ampoule. The ampoule was removed from the glovebox and $5 \mathrm{ml}$ of dry acetonitrile was added. The reaction mixture was stirred and heated to $100{ }^{\circ} \mathrm{C}$ for 17 hours in a silicone oil bath. Isolated yields were achieved by scaling up reactions and isolating products via column chromatography. The synthesis of $1 \mathrm{~g}$ was conducted in bromobenzene solvent at $150{ }^{\circ} \mathrm{C}$ for 17 hours rather than acetonitrile at $100{ }^{\circ} \mathrm{C}$ for both NMR tube and scaled up reactions. The synthesis of $1 \mathrm{~m}$ and $1 \mathrm{n}$ were conducted with 0.05 equivalents of 1,8-Diazabicyclo[5.4.0] undec-7-ene (DBU) catalyst, instead of DMAP.

a For both NMR scale and scaled up versions of the reactions, the reaction mixture is exposed to air upon addition of acetonitrile with no side reactivity or reduced conversion compared to an inert atmosphere of $\mathrm{N}_{2}$. It was, however, experimentally verified that drying of reagents and solvent is necessary in order to avoid side product formation of 4-hydroxy-2,3,56tetrafluoropyridine, identified by peaks at -101.8 and $-168.2 \mathrm{ppm}$ in the ${ }^{19} \mathrm{~F} \mathrm{NMR}$.

b Some scaled up reactions were conducted with one equivalent $(1.4 \mathrm{mmol})$ of carbonyl derivative instead of 10 equivalents, to aid separation of products from excess of carbonyl derivative. Isolation of products $\mathbf{1 b}, \mathbf{1} \mathbf{c}, \mathbf{1} \mathbf{j}, \mathbf{1} \mathbf{k}$ and $\mathbf{1}$ were performed with one equivalent of carbonyl derivative (Table S1). 


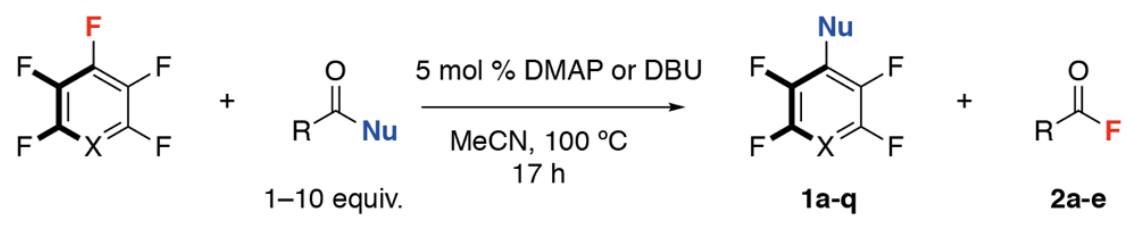

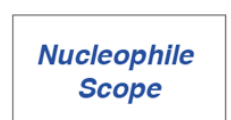<smiles>O=C(Oc1c(F)c(F)nc(F)c1F)c1ccccc1</smiles>

1a: $80 \pm 4 \%(56 \%)^{[a]} \quad$ 1b : $85 \pm 2 \%(53 \%)$ 2a $(\mathrm{R}=\mathrm{Ph}): 81 \pm 4 \%\left[{ }^{[\mathrm{b}]} \quad \mathbf{2 b}(\mathrm{R}=\mathrm{Me}): 99 \pm 4 \%\right.$ $73 \%$ isolated<smiles>Fc1nc(F)c(F)c(S)c1F</smiles>

1f : $99 \%(94 \%)$ 2b $(\mathrm{R}=\mathrm{Me}): 99 \%$<smiles>COc1c(F)c(F)c(OC(=O)c2ccccc2)c(F)c1F</smiles>

1k: $59 \pm 7 \%(36 \%)$ 2a $(\mathrm{R}=\mathrm{Ph}): 58 \pm 8 \%$<smiles>COc1c(F)c(F)c(OC(=O)c2ccccc2)c(F)c1F</smiles>

1 : $66 \pm 7 \%$ (42\%) 2a $(R=P h): 71 \pm 9 \%$<smiles>Fc1cc(F)c(F)c([SbH])c1F</smiles>

1m : $84 \pm 11 \%(68 \%)^{[c]}$ $2 b(R=M e): 47 \pm 15 \%$<smiles>Fc1nc(F)c(F)c(Cl)c1F</smiles><smiles>O=[N+]([O-])c1c(F)c(F)nc(F)c1F</smiles><smiles>N#Cc1c(F)c(F)nc(F)c1F</smiles>

\section{Fluoroarene} Scope

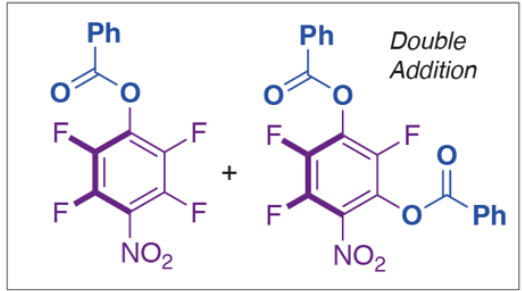

1p : $64 \pm 1 \%(37 \%) \quad$ 1p' : $21 \pm 5 \%(36 \%)$ 2a $(R=P h): 91 \pm 9 \%$<smiles>Fc1cc(F)c(F)c(S)c1</smiles>

In : $21 \pm 4 \%(28 \%)^{[c]}$ $2 b(R=M e): 10 \pm 1 \%$<smiles>CCCC(=O)Oc1c(F)c(F)nc(F)c1F</smiles><smiles>COC(=O)Oc1c(F)c(F)nc(F)c1F</smiles>

$1 e$ : $18 \%(20 \%)$ 2c $(\mathrm{R}=E-\mathrm{CH}=\mathrm{CHMe}): 2 \mathrm{~d}(\mathrm{R}=\mathrm{nonyl}): 66 \pm 2 \% \quad 2 \mathrm{e}(\mathrm{R}=\mathrm{OMe}): 39 \%$ $99 \pm 1 \%$

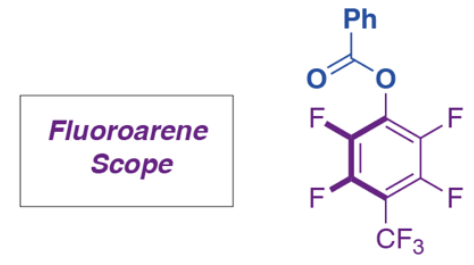

1j : $94 \pm 2 \%(66 \%)$ 2a $(\mathrm{R}=\mathrm{Ph}): 97 \pm 3 \%$

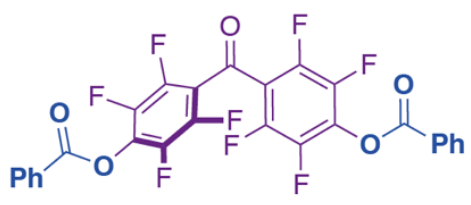

$$
10: 95 \pm 4 \%(30 \%)
$$

2a $(\mathrm{R}=\mathrm{Ph}): 97 \pm 1 \%$

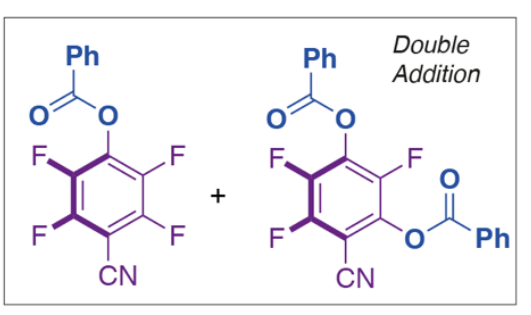

1q : $55 \pm 9 \%(32 \%) \quad$ 1q' : $40 \pm 9 \%(36 \%)$ 2a $(R=P h): 78 \pm 5 \%$

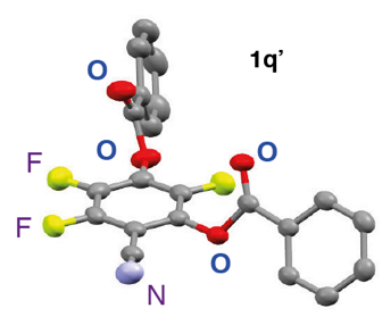

$X$-ray Structure

Table 1 Fluoride metathesis reaction of pentafluoropyridine with a range of different carbonyl derived functional groups.

Lower yields of fluoroarene product to carbonyl fluoride was observed in the cases of $1 \mathbf{e}$ and $\mathbf{1 h}$, which is attributed to partial decomposition of fluoroarenes product in these examples, for 1e likely by a decarboxylation pathway. Lower yields of product $2 b$ were observed in the cases of reaction $1 \mathrm{~m}$ and $1 \mathrm{n}$, owing to the high volatility of acyl fluoride $\left(20.8^{\circ} \mathrm{C}\right.$ boiling point). In all reactions that proceeded with lower conversion, remaining pentafluoropyridine was observed in the ${ }^{19} \mathrm{~F}$ NMR. 


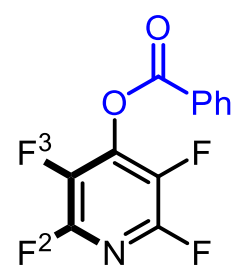

1a. 4-benzoic-2,3,5,6-tetrafluoropyridine: Isolation achieved by column chromatography in 7:3 n-pentane: dichloromethane eluent, $\mathrm{R}_{\mathrm{F}}=0.45$. Isolated as a crystalline white solid. ( 213 $\mathrm{mg}, 56.1 \%, 0.79 \mathrm{mmol})$.

${ }^{1} \mathrm{H}$ NMR $\left(\mathrm{C}_{6} \mathrm{D}_{6}, 400 \mathrm{MHz}\right): 7.93\left(\mathrm{~d}, 2 \mathrm{H},{ }^{3} \mathrm{~J}_{\mathrm{HH}}=7.2 \mathrm{~Hz}, \mathrm{o}-\mathrm{CH}\right), 7.08\left(\mathrm{t}, 1 \mathrm{H},{ }^{3} \mathrm{~J}_{\mathrm{HH}}=7.8 \mathrm{~Hz}, \mathrm{p}-\mathrm{CH}\right), 6.94$ $\left(\mathrm{t}, 2 \mathrm{H},{ }^{3} \mathrm{~J}_{\mathrm{HH}}=8.0 \mathrm{~Hz}, \mathrm{~m}-\mathrm{CH}\right)$.

${ }^{13} \mathrm{C}\left\{{ }^{1} \mathrm{H}\right\}$ NMR ( $\left.\mathrm{C}_{6} \mathrm{D}_{6}, 100 \mathrm{MHz}\right): 160.9$ (s, $\left.\underline{\mathrm{COPh}}\right), 144.6\left(\mathrm{dm},{ }^{1} \mathrm{~J}_{\mathrm{CF}}=243 \mathrm{~Hz}, \mathrm{CF}^{2}\right), 139.3(\mathrm{~m}, \underline{\mathrm{C}}-\mathrm{O})$, $137.6\left(\mathrm{dm},{ }^{1} \mathrm{~J}_{\mathrm{CF}}=262 \mathrm{~Hz}, \mathrm{CF}^{3}\right), 134.5\left(\mathrm{~s}, \mathrm{o}-\mathrm{C}_{\mathrm{Ph}}\right), 130.6\left(\mathrm{~s}, \mathrm{p}-\mathrm{C}_{\mathrm{Ph}}\right), 128.7\left(\mathrm{~s}, \mathrm{~m}-\mathrm{C}_{\mathrm{Ph}}\right), 126.2\left(\mathrm{~s}, \mathrm{i}-\mathrm{C}_{\mathrm{Ph}}\right)$

${ }^{19} \mathrm{~F}$ NMR $\left(\mathrm{C}_{6} \mathrm{D}_{6}, 100 \mathrm{MHz}\right):-88.9\left(\mathrm{~m}, \mathrm{CF}^{2}\right),-152.5\left(\mathrm{~m}, \mathrm{CF}^{3}\right)$.

IR ( $\left.\mathrm{cm}^{-1}\right): 1770$ (C=O str), 1282 (C-O str), 1234 (C-O str).

HRMS (APCL): m/z: $\mathrm{M}+$ Calcd for $\mathrm{C}_{12} \mathrm{H}_{5} \mathrm{NO}_{2} \mathrm{~F}_{4}$ 271.0256; molecular ion not observed. 
<smiles>[Y6]C(=O)Oc1c(F)c(F)nc(F)c1F</smiles>

1b. 4-acetyl-2,3,5,6-tetrafluoropyridine: Isolation achieved by column chromatography in 4: 1 pentane: dichloromethane to yield product as a colourless oil. $R_{F}=0.65$. (155 mg, $52.5 \%$, $0.74 \mathrm{mmol})$.

${ }^{1} \mathrm{H} \operatorname{NMR}\left(\mathrm{C}_{6} \mathrm{D}_{6}, 400 \mathrm{MHz}\right): 4.34\left(\mathrm{~s}, 3 \mathrm{H}, \mathrm{CH}_{3}\right)$.

${ }^{13} \mathrm{C}\left\{{ }^{1} \mathrm{H}\right\} \mathrm{NMR}\left(\mathrm{C}_{6} \mathrm{D}_{6}, 100 \mathrm{MHz}\right): 164.3\left(\mathrm{~s}, \underline{\mathrm{COCH}}_{3}\right), 144.5\left(\mathrm{dm},{ }^{1} \mathrm{~J}_{\mathrm{CF}}=244 \mathrm{~Hz}, \mathrm{CF}^{2}\right), 137.3\left(\mathrm{dm},{ }^{1} \mathrm{~J}_{\mathrm{CF}}\right.$ $\left.=262 \mathrm{~Hz}, \mathrm{CF}^{3}\right), 18.3\left(\mathrm{~s}, \mathrm{CH}_{3}\right)$. Ipso-carbon resonance not observed

${ }^{19} \mathrm{~F} \operatorname{NMR}\left(\mathrm{C}_{6} \mathrm{D}_{6}, 100 \mathrm{MHz}\right):-89.1\left(\mathrm{~m}, \mathrm{CF}^{2}\right),-153.3\left(\mathrm{~m}, \mathrm{CF}^{3}\right)$.

HRMS (APCL) m/z: [M + H] ${ }^{+}$Calcd for $\mathrm{C}_{7} \mathrm{H}_{3} \mathrm{~F}_{4} \mathrm{NO}_{2}$ 210.0103; Found 210.0517. 
<smiles>C/C=C/C(=O)Oc1c(F)c(F)nc(F)c1F</smiles>

1c. 4-Crotoic-2,3,5,6-tetrafluoropyridine: Isolation achieved by column chromatography in 9: $1 \mathrm{n}$-hexane: dichloromethane to yield product as a yellow oil. $\mathrm{R}_{\mathrm{F}}=0.35$. (153 mg, 46.2 \%, 0.65 mmol).

${ }^{1} \mathrm{H}$ NMR $\left(\mathrm{C}_{6} \mathrm{D}_{6}, 400 \mathrm{MHz}\right): 6.87\left(\mathrm{dq}, 1 \mathrm{H}, 3^{3} \mathrm{~J}_{\mathrm{HH}}=16.2 \mathrm{~Hz},{ }^{3} \mathrm{~J}_{\mathrm{HH}}=7.1 \mathrm{~Hz}, \mathrm{CH}=\mathrm{C}_{\underline{H}} \mathrm{CH}_{3}\right), 5.55(\mathrm{~d}, 1 \mathrm{H}$, $\left.{ }^{3} \mathrm{~J}_{\mathrm{HH}}=16.2 \mathrm{~Hz}, \mathrm{CH}=\mathrm{CHCH}_{3}\right), 1.25\left(\mathrm{~d}, 3 \mathrm{H}, 3^{3} \mathrm{~J}_{\mathrm{HH}}=7.1 \mathrm{~Hz}, \mathrm{CH}=\mathrm{CHC}_{\underline{H}}\right)$.

${ }^{13} \mathrm{C}\left\{{ }^{1} \mathrm{H}\right\}$ NMR $\left.\left(\mathrm{C}_{6} \mathrm{D}_{6}, 100 \mathrm{MHz}\right): 160.0(\mathrm{~s}, \mathrm{O} \underline{\mathrm{COOCHCHCH}})_{3}\right), 151.5\left(\mathrm{~s}, \mathrm{OCOCH} \underline{\mathrm{C}} \mathrm{HCH}_{3}\right), 144.52(\mathrm{dm}$, $\left.\left.{ }^{1} \mathrm{~J}_{\mathrm{CF}}=243 \mathrm{~Hz}, \mathrm{CF}^{2}\right), 139.4(\mathrm{~m}, \mathrm{i}-\mathrm{C}), 137.7\left(\mathrm{dm},{ }^{1} \mathrm{~J}_{\mathrm{CF}}=268 \mathrm{~Hz}, \mathrm{CF}^{3}\right), 118.4(\mathrm{~s}, 1 \mathrm{C}, \mathrm{OCO} \underline{\mathrm{CHCHCH}})_{3}\right)$, $17.6\left(\mathrm{~s}, 1 \mathrm{C}, \mathrm{CH}_{3}\right)$.

${ }^{19} \mathrm{~F}$ NMR $\left(\mathrm{C}_{6} \mathrm{D}_{6}, 100 \mathrm{MHz}\right):-89.3\left(\mathrm{~m}, \mathrm{CF}^{2}\right),-153.0\left(\mathrm{~m}, \mathrm{CF}^{3}\right)$.

IR (cm-1): 1763 (C=O str), 1297 (C-O str), 1200 (C-O str).

HRMS (APCL) m/z: [M + H] $]^{+}$Calcd for $\mathrm{C}_{9} \mathrm{H}_{6} \mathrm{~F}_{4} \mathrm{NO}_{2} 236.0329$; Found 236.0332 . 


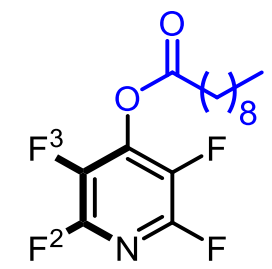

1d. 4-decanoic-2,3,5,6-tetrafluoropyridine: (98 mg, $22.6 \%, 0.32 \mathrm{mmol}$ ). Isolation achieved by column chromatography in 9: $1 \mathrm{n}$-hexane: dichloromethane. Isolated as an amorphous white solid. $R_{F}=0.27$

${ }^{1} \mathrm{H}$ NMR $\left(\mathrm{C}_{6} \mathrm{D}_{6}, 400 \mathrm{MHz}\right): 2.06\left(\mathrm{t}, 2 \mathrm{H},{ }^{3} \mathrm{~J}_{\mathrm{HH}}=7.6 \mathrm{~Hz}, \mathrm{COCH}_{2}\right.$ ), 1.35-1.15 (series of $\left.\mathrm{m}, 14 \mathrm{H}\right), 0.92$ $\left(\mathrm{t}, 3 \mathrm{H},{ }^{3} \mathrm{~J}_{\mathrm{HH}}=7.1 \mathrm{~Hz}, \mathrm{CH}_{3}\right)$.

${ }^{13} \mathrm{C}\left\{{ }^{1} \mathrm{H}\right\}$ NMR ( $\left.\mathrm{C}_{6} \mathrm{D}_{6}, 100 \mathrm{MHz}\right): 167.6(\mathrm{~s}, \underline{\mathrm{COR}}), 144.5\left(\mathrm{dm},{ }^{1} \mathrm{~J}_{\mathrm{CF}}=224 \mathrm{~Hz}, \mathrm{CF}^{2}\right), 137.2\left(\mathrm{dm},{ }^{1} \mathrm{~J}_{\mathrm{CF}}=\right.$ $247 \mathrm{~Hz}, \mathrm{CF}^{3}$ ), $32.7\left(\mathrm{~s}, \mathrm{CH}_{2}\right), 32.0\left(\mathrm{~s}, \mathrm{CH}_{2}\right), 29.7\left(\mathrm{~d}, \mathrm{~J}_{\mathrm{CF}}=5.8 \mathrm{~Hz}, \mathrm{CH}_{2}\right), 29.4\left(\mathrm{~d}, \mathrm{~J}_{\mathrm{CF}}=3.6 \mathrm{~Hz}, \mathrm{CH}_{2}\right.$ ), $29.1\left(\mathrm{~s}, \mathrm{CH}_{2}\right), 28.6\left(\mathrm{~s}, \mathrm{CH}_{2}\right), 24.4\left(\mathrm{~s}, \mathrm{CH}_{2}\right), 22.8\left(\mathrm{~s}, \mathrm{CH}_{2}\right), 14.0\left(\mathrm{~s}, \mathrm{CH}_{3}\right)$. Ipso carbon not observed.

${ }^{19} \mathrm{~F}$ NMR $\left(\mathrm{C}_{6} \mathrm{D}_{6}, 100 \mathrm{MHz}\right):-88.9\left(\mathrm{~m}, \mathrm{CF}^{2}\right),-153.0\left(\mathrm{~m}, \mathrm{CF}^{3}\right)$.

IR (cm $\left.{ }^{-1}\right): 1797$ (C=O str), 1275 (C-O str).

HRMS (APCL) m/z: [M + H] ${ }^{+}$Calcd for $\mathrm{C}_{14} \mathrm{H}_{19} \mathrm{NO}_{2} \mathrm{~F}_{4}$ 309.1352; Molecular lon not observed. 
<smiles>COC(=O)Oc1c(F)c(F)nc(F)c1F</smiles>

1e. methyl-2,3,4,5-tetrafluoropyridin-4-ylcarbonate: Isolation achieved by column chromatography in 9:1 $\mathrm{n}$-hexane : ethyl acetate, $\mathrm{R}_{\mathrm{F}}=0.56$. Isolated as a colourless oil. (61.8 $\mathrm{mg}, 19.6 \%, 0.27 \mathrm{mmol})$.

${ }^{1} \mathrm{H} \operatorname{NMR}\left(\mathrm{C}_{6} \mathrm{D}_{6}, 400 \mathrm{MHz}\right): 3.04\left(\mathrm{~s}, 1 \mathrm{H}, \mathrm{CH}_{3}\right)$.

${ }^{13} \mathrm{C}\left\{{ }^{1} \mathrm{H}\right\}$ NMR $\left(\mathrm{C}_{6} \mathrm{D}_{6}, 100 \mathrm{MHz}\right): 150.2(\mathrm{~s}, \underline{\mathrm{COMe}}), 144.4\left(\mathrm{dm},{ }^{1} \mathrm{~J}_{\mathrm{CF}}=247 \mathrm{~Hz}, \mathrm{CF}^{2}\right), 138.2\left(\mathrm{dm},{ }^{1} \mathrm{~J}_{\mathrm{CF}}\right.$ $\left.=242 \mathrm{~Hz}, \mathrm{CF}^{3}\right), 56.1\left(\mathrm{~s}, \mathrm{CH}_{3}\right)$. Ipso carbon not observed.

${ }^{19} \mathrm{~F} N M R\left(\mathrm{C}_{6} \mathrm{D}_{6}, 100 \mathrm{MHz}\right):-90.9\left(\mathrm{~m}, \mathrm{CF}^{2}\right),-154.7\left(\mathrm{~m}, \mathrm{CF}^{3}\right)$.

HRMS (APCL) m/z: [M + H] ${ }^{+}$Calcd for $\mathrm{C}_{7} \mathrm{H}_{3} \mathrm{~F}_{4} \mathrm{NO}_{3}$ 226.0204; Found 226.1318.

Minor decomposition to 4-hydroxy-2,3,5,6-tetrafluoropyridine was observed over the course of the reaction, noted by the formation of peaks at -101.8 and $-168.2 \mathrm{ppm}$ in the ${ }^{19} \mathrm{~F}$ NMR spectrum. 


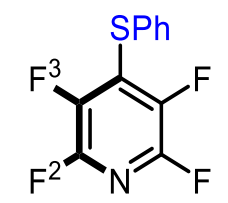

1f. 4-phenylthio-2,3,5,6-tetrafluoropyridine: Isolation achieved by column chromatography in 9:1 pentane : dichloromethane to yield a colourless oil, $R_{F}=0.40$. Isolated as a crystalline white solid. (341 mg, $94.1 \%, 1.32 \mathrm{mmol}$ ). ${ }^{[2]}$

${ }^{1} \mathrm{H} \mathrm{NMR}\left(\mathrm{C}_{6} \mathrm{D}_{6}, 400 \mathrm{MHz}\right): 7.10\left(\mathrm{~d}, 2 \mathrm{H},{ }^{3} \mathrm{~J}_{\mathrm{HH}}=6.7 \mathrm{~Hz}, \mathrm{o}-\mathrm{CH}\right), 6.90(\mathrm{~s}, 1 \mathrm{H}, \mathrm{p}-\mathrm{CH}), 6.88-6.83(\mathrm{~m}, 2 \mathrm{H}$, $\mathrm{m}-\mathrm{CH})$.

${ }^{13} \mathrm{C}\left\{{ }^{1} \mathrm{H}\right\} \mathrm{NMR}\left(\mathrm{C}_{6} \mathrm{D}_{6}, 100 \mathrm{MHz}\right): 143.3\left(\mathrm{dm},{ }^{1} \mathrm{~J}_{\mathrm{CF}}=228 \mathrm{~Hz}, \mathrm{CF}^{2}\right), 140.8\left(\mathrm{dm},{ }^{1} \mathrm{~J}_{\mathrm{CF}}=232 \mathrm{~Hz}, \mathrm{CF}^{3}\right)$, $132.5\left(\mathrm{~s}, \mathrm{o}-\mathrm{C}_{\mathrm{Ph}}\right), 129.3\left(\mathrm{~s}, \mathrm{p}-\mathrm{C}_{\mathrm{Ph}}\right), 128.9\left(\mathrm{~s}, \mathrm{~m}-\mathrm{C}_{\mathrm{Ph}}\right)$. Ipso-carbon resonances not observed.

${ }^{19} \mathrm{~F}$ NMR $\left(\mathrm{C}_{6} \mathrm{D}_{6}, 100 \mathrm{MHz}\right):-90.9\left(\mathrm{~m}, \mathrm{CF}^{2}\right),-136.6\left(\mathrm{~m}, \mathrm{CF}{ }^{3}\right)$.

HRMS (APCL) m/z: [M + H] ${ }^{+}$Calcd for $\mathrm{C}_{12} \mathrm{H}_{3} \mathrm{~F}_{4} \mathrm{NO}_{2} 242.0518$; Found 242.0620. 


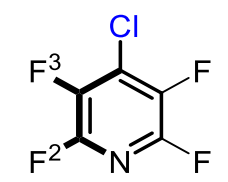

1g. 4-chloro-2,3,5,6-tetrafluoropyridine:

Product not isolated, NMR yield $=51 \%$ based on ${ }^{19} \mathrm{~F}$ NMR spectroscopy.

${ }^{19} \mathrm{~F}$ NMR $\left(\mathrm{C}_{6} \mathrm{D}_{6}, 100 \mathrm{MHz}\right)$ : -90.6 $\left(\mathrm{m}, \mathrm{CF}^{2}\right),-142.6\left(\mathrm{~m}, \mathrm{CF}^{3}\right)$. Data are in close agreement with the current literature. ${ }^{[\mathrm{S} 3]}$ 


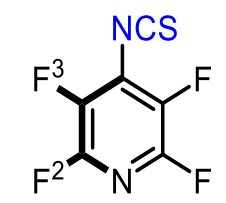

1h. 4-isothiocyanato-2,3,5,6-tetrafluoropyridine: Isolation achieved by column chromatography in 9:1 $\mathrm{n}$-hexane : ethyl-acetate as a yellow oil. $\mathrm{R}_{\mathrm{F}}=0.44$. $(75.4 \mathrm{mg}, 25.9 \%$, $0.36 \mathrm{mmol}){ }^{*}$

${ }^{13} \mathrm{C}\left\{{ }^{1} \mathrm{H}\right\} \operatorname{NMR}\left(\mathrm{C}_{6} \mathrm{D}_{6}, 100 \mathrm{MHz}\right): 148.1(\mathrm{~s}, \mathrm{~N} \underline{\mathrm{CS}}), 143.1\left(\mathrm{dm},{ }^{1} \mathrm{~J}_{\mathrm{CF}}=237 \mathrm{~Hz}, \mathrm{CF}^{3}\right), 140.1\left(\mathrm{dm},{ }^{1} \mathrm{~J}_{\mathrm{CF}}=\right.$ $\left.240 \mathrm{~Hz}, \mathrm{CF}^{2}\right), 128.5$ (s, $\left.\underline{\mathrm{C}}-\mathrm{NCS}\right)$.

${ }^{19} \mathrm{~F}$ NMR $\left(\mathrm{C}_{6} \mathrm{D}_{6}, 100 \mathrm{MHz}\right):-91.4\left(\mathrm{~m}, \mathrm{CF}^{2}\right),-133.9\left(\mathrm{~m}, \mathrm{CF}^{3}\right)$.

IR (cm-1): 1916 (NCS str).

HRMS (APCL) m/z: [M + H] ${ }^{+}$Calcd for $\mathrm{C}_{5} \mathrm{~F}_{4} \mathrm{NS}$ 181.9850; Found 181.9687.

*The $\mathrm{SCN}^{-}$anion is ambiphilic and can potentially perform $\mathrm{S}_{\mathrm{N}} \mathrm{Ar}$ from either the $\mathrm{S}^{-}$or $\mathrm{N}^{-}$ positions. 4-thiocyanato-2,3,5,6-tetrafluoropyridine is characterised by a nitrile carbon environment at approximately $110 \mathrm{ppm}$, and a CN stretch at approximately $3150 \mathrm{~cm}^{-1}$. ${ }^{[S 4]} 4$ isothiocyanato-2,3,5,6-tetrafluopyridine is characterised by a NCS carbon environment at $148.1 \mathrm{ppm}$ and a NCS stretch at $1916 \mathrm{~cm}^{-1}$. [55] 


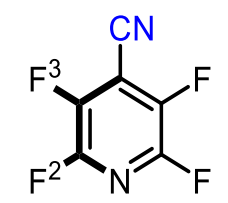

1i. 4-cyano-2,3,5,6-tetrafluoropyridine: Isolation achieved by column chromatography in 9:1 $\mathrm{n}$-pentane: dichloromethane. Isolated as a colourless oil. $\mathrm{R}_{\mathrm{F}}=0.57$. (45 mg, 18.2\%, 0.26 $\mathrm{mmol}) .{ }^{[\mathrm{s} 6]}$

${ }^{13} \mathrm{C}\left\{{ }^{1} \mathrm{H}\right\}$ NMR $\left(\mathrm{C}_{6} \mathrm{D}_{6}, 100 \mathrm{MHz}\right): 143.6\left(\mathrm{~s}, \mathrm{CF}^{2}\right), 136.4\left(\mathrm{~s}, \mathrm{CF}^{3}\right), 112.9$ (s, $\left.\underline{\mathrm{CN}}\right), 110.0$ (s, i-C).

${ }^{19} \mathrm{~F}$ NMR $\left(\mathrm{C}_{6} \mathrm{D}_{6}, 100 \mathrm{MHz}\right):-88.6\left(\mathrm{~m}, \mathrm{CF}^{2}\right),-153.2\left(\mathrm{~m}, \mathrm{CF}^{3}\right)$.

IR ( $\left.\mathrm{cm}^{-1}\right): 3092$ (N-H amide str), 1685 (C=O amide str) - suggests nitrile hydrolysis to amide.* HRMS (APCL) m/z: [M+ $\left.\mathrm{H}_{2} \mathrm{O}-\mathrm{NH}_{2}\right]^{+}$Calcd for $\mathrm{C}_{6} \mathrm{~N}_{2} \mathrm{~F}_{4}$ 177.9916; Found 178.0232.

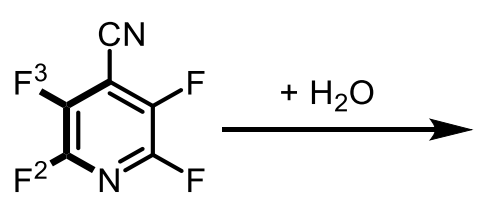<smiles>NC(=O)c1c(F)c(F)nc(F)c1F</smiles>

*It is proposed that hydrolysis of species $\mathbf{1 i}$ occurred before IR and mass spectroscopy were taken, consistent with the observation of amide $\mathrm{N}-\mathrm{H}$ and $\mathrm{C}=\mathrm{O}$ stretches in the IR spectrum, and the detection of $\mathrm{C}_{6} \mathrm{NFO}_{4}{ }^{+}$ion in mass spectroscopy. 
<smiles>O=C(Oc1c(F)c(F)c(C(F)(F)F)c(F)c1F)c1ccccc1</smiles>

1j 4-benzoic-2,3,5,6-tetrafluorooctofluorotoluene: Isolation achieved by column chromatography in 2:1 hexane: dichloromethane. Isolated as a crystalline white solid. $\mathrm{R}_{\mathrm{F}}=$ 0.40 (309 mg, 65.8\%, $0.92 \mathrm{mmol}$ ).

${ }^{1} \mathrm{H} \mathrm{NMR}\left(\mathrm{C}_{6} \mathrm{D}_{6}, 400 \mathrm{MHz}\right): 7.99\left(\mathrm{~d}, 2 \mathrm{H},{ }^{3} \mathrm{~J}_{\mathrm{HH}}=7.9 \mathrm{~Hz}, \mathrm{o}-\mathrm{CH}\right), 7.08\left(\mathrm{t}, 1 \mathrm{H},{ }^{3} \mathrm{~J}_{\mathrm{HH}}=7.5 \mathrm{~Hz}, \mathrm{p}-\mathrm{CH}\right), 6.95$ $\left(\mathrm{t}, 2 \mathrm{H},{ }^{3} \mathrm{~J}_{\mathrm{HH}}=7.6 \mathrm{~Hz}, \mathrm{~m}-\mathrm{CH}\right)$.

${ }^{13} \mathrm{C}\left\{{ }^{1} \mathrm{H}\right\}$ NMR $\left(\mathrm{C}_{6} \mathrm{D}_{6}, 100 \mathrm{MHz}\right): 161.6(\mathrm{~s}, \underline{\mathrm{COPh}}), 145.6\left(\mathrm{dm},{ }^{1} \mathrm{~J}_{\mathrm{CF}}=264 \mathrm{~Hz}, \mathrm{CF}^{2}\right), 142.4\left(\mathrm{dm},{ }^{1} \mathrm{~J}_{\mathrm{CF}}\right.$

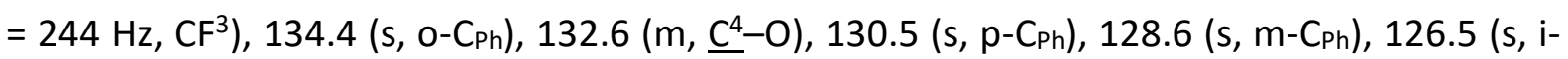

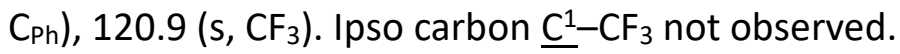

${ }^{19} \mathrm{~F} N M R\left(\mathrm{C}_{6} \mathrm{D}_{6}, 100 \mathrm{MHz}\right):-55.8\left(\mathrm{t},{ }^{2} \mathrm{~J}_{\mathrm{FF}}=21.9 \mathrm{~Hz}, \mathrm{CF}_{3}\right),-141.0\left(\mathrm{~m}, \mathrm{CF}^{2}\right),-150.9\left(\mathrm{~m}, \mathrm{CF}^{3}\right)$.

IR (cm-1): 1767 (C=O str), 1271 (C-O str), 1208 (C-O str).

HRMS (APCL) m/z: [M + H] $]^{+}$Calcd for $\mathrm{C}_{14} \mathrm{H}_{5} \mathrm{~F}_{7} \mathrm{O}_{2}$ 339.0251; Found 339.0249. 
<smiles>CC(=O)c1c(F)c(F)c(OC(=O)c2ccccc2)c(F)c1F</smiles>

1k, 4-benzoic-2,3,5,6-tetrafluoroacetophenone Isolation achieved by column chromatography in 9: 1 hexane: dichloromethane to yield product as a white crystalline solid. $\mathrm{R}_{\mathrm{F}}=0.50$. (159 mg, $\left.36.1 \%, 0.51 \mathrm{mmol}\right)$. Recrystallized for XRD characterisation by diffusion of $\mathrm{n}$-pentane into ethyl acetate solution at room temperature.

${ }^{1} \mathrm{H} \mathrm{NMR}\left(\mathrm{C}_{6} \mathrm{D}_{6}, 400 \mathrm{MHz}\right): 8.02\left(\mathrm{~d}, 2 \mathrm{H},{ }^{3} \mathrm{~J}_{\mathrm{HH}}=8.1 \mathrm{~Hz}, \mathrm{o}-\mathrm{CH}\right), 7.08\left(\mathrm{t}, 1 \mathrm{H},{ }^{3} \mathrm{~J}_{\mathrm{HH}}=7.6 \mathrm{~Hz}, \mathrm{p}-\mathrm{CH}\right), 6.95$ $\left(\mathrm{t}, 2 \mathrm{H},{ }^{3} \mathrm{~J}_{\mathrm{HH}}=8.2 \mathrm{~Hz}, \mathrm{~m}-\mathrm{CH}\right), 1.94\left(\mathrm{~m}, 3 \mathrm{H}, \mathrm{COCH}_{3}\right)$.

${ }^{13} \mathrm{C}\left\{{ }^{1} \mathrm{H}\right\}$ NMR $\left(\mathrm{C}_{6} \mathrm{D}_{6}, 100 \mathrm{MHz}\right): 189.7(\mathrm{~s}, \underline{\mathrm{COCH}} 3), 162.1(\mathrm{~s}, \underline{\mathrm{COPh}}), 150.4\left(\mathrm{dm},{ }^{1} \mathrm{~J}_{\mathrm{CF}}=260 \mathrm{~Hz}\right)$, $145.6\left(\mathrm{dm},{ }^{1} \mathrm{~J}_{\mathrm{CF}}=256 \mathrm{~Hz}\right), 134.2\left(\mathrm{~s}, \mathrm{o}-\mathrm{C}_{\mathrm{Ph}}\right), 130.5\left(\mathrm{~s}, \mathrm{p}-\mathrm{C}_{\mathrm{Ph}}\right), 128.6\left(\mathrm{~s}, \mathrm{~m}-\mathrm{C}_{\mathrm{Ph}}\right), 126.8\left(\mathrm{~s}, \mathrm{i}-\mathrm{C}_{\mathrm{Ph}}\right), 31.4$ $\left(\mathrm{s}, \mathrm{CH}_{3}\right)$. Remaining ipso carbon resonances not observed.

${ }^{19} \mathrm{~F} \operatorname{NMR}\left(\mathrm{C}_{6} \mathrm{D}_{6}, 100 \mathrm{MHz}\right):-142.3\left(\mathrm{~m}, \mathrm{CF}^{2}\right),-152.3\left(\mathrm{~m}, \mathrm{CF}^{3}\right)$.

IR ( $\left.\mathrm{cm}^{-1}\right)$ : 1759 (C=O ester str), 1700 (C=O ketone str), 1312 (C-O str), 1241 (C-O str).

HRMS (APCL) m/z: [M + H] $]^{+}$Calcd for $\mathrm{C}_{15} \mathrm{H}_{9} \mathrm{~F}_{4} \mathrm{O}_{3}$ 313.0482; Found 313.0479. 
<smiles>COC(=O)c1c(F)c(F)c(OC(=O)c2ccccc2)c(F)c1F</smiles>

11, 4-benzoic-2,3,5,6-tetrafluoromethylbenzoate Isolation achieved by column chromatography in 95:5 pentane: ethyl acetate, to yield product as a white crystalline solid. $\mathrm{R}_{\mathrm{F}}=0.46$ (197 mg, $\left.42.4 \%, 0.60 \mathrm{mmol}\right)$.

${ }^{1} \mathrm{H}$ NMR $\left(\mathrm{C}_{6} \mathrm{D}_{6}, 400 \mathrm{MHz}\right): 8.00\left(\mathrm{~d}, 2 \mathrm{H},{ }^{3} \mathrm{~J}_{\mathrm{HH}}=7.1 \mathrm{~Hz}, \mathrm{o}-\mathrm{CH}\right), 7.04\left(\mathrm{t}, 1 \mathrm{H},{ }^{3} \mathrm{~J}_{\mathrm{HH}}=7.3 \mathrm{~Hz}, \mathrm{p}-\mathrm{CH}\right), 6.93$ $\left(\mathrm{t}, 2 \mathrm{H},{ }^{3} \mathrm{~J}_{\mathrm{HH}}=7.6 \mathrm{~Hz}, \mathrm{~m}-\mathrm{CH}\right), 3.33\left(\mathrm{~s}, 3 \mathrm{H}, \mathrm{CH}_{3}\right)$.

${ }^{13} \mathrm{C}\left\{{ }^{1} \mathrm{H}\right\}$ NMR ( $\left.\mathrm{C}_{6} \mathrm{D}_{6}, 100 \mathrm{MHz}\right): 161.9$ (s, $\left.\mathrm{COPh}\right), 145.5\left(\mathrm{~m}, \mathrm{CF}^{2}\right), 141.7\left(\mathrm{~m}, \mathrm{CF}^{3}\right), 134.2$ (s, o- $\left.\mathrm{C}_{\mathrm{Ph}}\right)$,

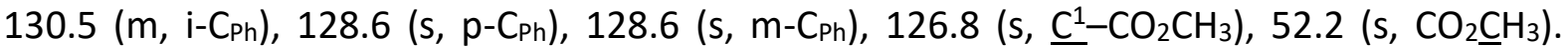
Remaining ipso carbon resonances not observed.

${ }^{19} \mathrm{~F}$ NMR $\left(\mathrm{C}_{6} \mathrm{D}_{6}, 100 \mathrm{MHz}\right):-146.9\left(\mathrm{~m}, \mathrm{CF}^{2}\right),-149.9\left(\mathrm{~m}, \mathrm{CF}^{3}\right)$

IR $\left(\mathrm{cm}^{-1}\right)$ : 1767 ( $\mathrm{C}=\mathrm{O}$ ester str), 1730 ( $\mathrm{C}=\mathrm{O}$ acid str), 1323 (C-O acid str), 1219 (C-O ester str), 1103 (C-O ester str)

HRMS (APCL) m/z: [M + H] $]^{+}$Calcd for $\mathrm{C}_{15} \mathrm{H}_{9} \mathrm{~F}_{4} \mathrm{O}_{4}$ 329.0431; Found 329.0432. 


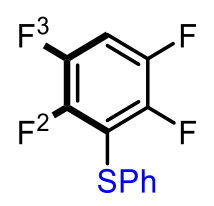

1m, phenyl-2,3,5,6-tetrafluorophenylsulfane: Isolation achieved by column chromatography in 19:1 $\mathrm{n}$-hexane : dichloromethane to yield product as a colourless oil. $\mathrm{R}_{\mathrm{F}}=0.69(244.2 \mathrm{mg}$, $67.6 \%, 0.95 \mathrm{mmol}) .{ }^{[57]}$

${ }^{1} \mathrm{H}$ NMR $\left(\mathrm{C}_{6} \mathrm{D}_{6}, 400 \mathrm{MHz}\right): 7.20-7.17(\mathrm{~m}, 2 \mathrm{H}, \mathrm{m}-\mathrm{CH}), 6.85(\mathrm{~m}, 1 \mathrm{H}, \mathrm{p}-\mathrm{CH}), 6.83(\mathrm{~m}, 2 \mathrm{H}, \mathrm{o}-\mathrm{CH})$, 6.11-6.03 $(\mathrm{m}, 1 \mathrm{H}, \mathrm{CH})$.

${ }^{13} \mathrm{C}\left\{{ }^{1} \mathrm{H}\right\} \operatorname{NMR}\left(\mathrm{C}_{6} \mathrm{D}_{6}, 100 \mathrm{MHz}\right): 147.5\left(\mathrm{dm},{ }^{1} \mathrm{~J}_{\mathrm{CF}}=102 \mathrm{~Hz}, \mathrm{CF}^{2}\right), 145.1,\left(\mathrm{dm},{ }^{1} \mathrm{~J}_{\mathrm{CF}}=108 \mathrm{~Hz}, \mathrm{CF}^{3}\right)$, 134.4 (s, i- $\left.C_{P h}\right), 130.5$ (s, o- $\left.C_{P h}\right), 129.2$ (s, m- $\left.C_{P h}\right), 128.9$ (s, p- $\left.C_{P h}\right), 106.6$ (t, ${ }^{2} \mathrm{JFF}_{\mathrm{CF}}=27.5 \mathrm{~Hz}, \mathrm{C}^{4}-\mathrm{H}$ ). Ipso carbon (C-S) not observed.

${ }^{19} \mathrm{~F} \operatorname{NMR}\left(\mathrm{C}_{6} \mathrm{D}_{6}, 100 \mathrm{MHz}\right): 134.4\left(\mathrm{~m}, \mathrm{CF}^{2}\right),-138.9\left(\mathrm{~m}, \mathrm{CF}^{3}\right)$

HRMS (APCL) m/z: [M + H] Calcd for $\mathrm{C}_{12} \mathrm{H}_{6} \mathrm{~F}_{4} \mathrm{~S}$ 259.0191; Found 259.0197. 


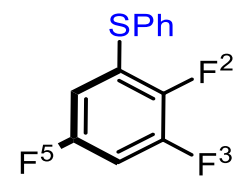

1n, phenyl-2,3,5-trifluorophenylsulfane: Isolation achieved by column chromatography in 19:1 $\mathrm{n}$-hexane : dichloromethane to yield product as a colourless oil. $\mathrm{R}_{\mathrm{F}}=0.69$ (96.8 $\mathrm{mg}, 28.7$ $\%, 0.40 \mathrm{mmol}) .{ }^{[\mathrm{s}]}$

${ }^{1} \mathrm{H} \mathrm{NMR}\left(\mathrm{C}_{6} \mathrm{D}_{6}, 400 \mathrm{MHz}\right): 7.17\left(\mathrm{~d}, 1 \mathrm{H},{ }^{3} \mathrm{~J}_{\mathrm{HH}}=2.0 \mathrm{~Hz}, \mathrm{p}-\mathrm{CH}\right), 6.88\left(\mathrm{~d}, 2 \mathrm{H},{ }^{3} \mathrm{~J}_{\mathrm{HH}}=1.8 \mathrm{~Hz}, \mathrm{~m}-\mathrm{CH}\right)$, $6.87\left(\mathrm{~d}, 2 \mathrm{H},{ }^{3} \mathrm{~J}_{\mathrm{HH}}=2.1 \mathrm{~Hz}, \mathrm{o}-\mathrm{CH}\right), 6.33-6.28\left(\mathrm{~m}, 1 \mathrm{H}, \mathrm{CH}^{6}\right), 6.14-6.07\left(\mathrm{~m}, 1 \mathrm{H}, \mathrm{CH}^{4}\right)$.

${ }^{13} \mathrm{C}\left\{{ }^{1} \mathrm{H}\right\}$ NMR $\left(\mathrm{C}_{6} \mathrm{D}_{6}, 100 \mathrm{MHz}\right): 157.6\left(\mathrm{~d},{ }^{1} \mathrm{~J}_{\mathrm{CF}}=248 \mathrm{~Hz}, \mathrm{CF}^{2}\right), 201.9\left(\mathrm{~d},{ }^{1} \mathrm{~J}_{\mathrm{CF}}=248 \mathrm{~Hz}, \mathrm{CF}^{3}\right), 182.2$

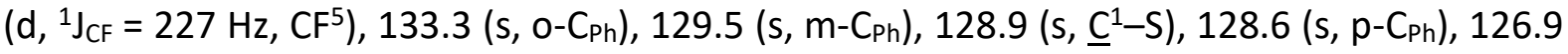
$\left(\mathrm{s}, 1 \mathrm{C}, \mathrm{i}-\mathrm{C}_{\mathrm{Ph}}\right), 111.7-111.5\left(\mathrm{~m}, \mathrm{C}^{6}-\mathrm{H}\right), 103.5-103.0\left(\mathrm{~m}, \mathrm{C}^{4}-\mathrm{H}\right)$.

${ }^{19} \mathrm{~F}$ NMR $\left(\mathrm{C}_{6} \mathrm{D}_{6}, 100 \mathrm{MHz}\right):-115.2\left(\mathrm{~m}, 1 \mathrm{~F}, \mathrm{CF}^{5}\right),-134.2\left(\mathrm{~m}, \mathrm{CF}{ }^{3}\right),-141.6\left(\mathrm{~m}, \mathrm{CF}^{2}\right)$.

HRMS (APCL) m/z: [M + H] ${ }^{+}$Calcd for $\mathrm{C}_{12} \mathrm{H}_{7} \mathrm{~F}_{3} \mathrm{~S}^{+}$241.0293; Found 241.0289. 
<smiles>O=C(Oc1c(F)c(F)c(C(=O)c2c(F)c(F)c(OC(=O)c3ccccc3)c(F)c2F)c(F)c1F)c1ccccc1</smiles>

10, 4,4-dibenzoic octofluorobenzophenone: Isolation achieved by column chromatography in 9:1 hexane:dichloromethane to yield product as a white crystalline solid. $R_{F}=0.29$ (236 mg, $29.8 \%, 0.42 \mathrm{mmol})$.

${ }^{1} \mathrm{H} \mathrm{NMR}\left(\mathrm{C}_{6} \mathrm{D}_{6}, 400 \mathrm{MHz}\right): 8.00\left(\mathrm{~d}, 4 \mathrm{H},{ }^{3} \mathrm{~J}_{\mathrm{HH}}=8.1 \mathrm{~Hz}, \mathrm{o}-\mathrm{CH}\right), 7.07\left(\mathrm{t}, 2 \mathrm{H},{ }^{3} \mathrm{~J}_{\mathrm{HH}}=7.8 \mathrm{~Hz}, \mathrm{p}-\mathrm{CH}\right), 6.94$ $\left(\mathrm{t}, 4 \mathrm{H},{ }^{3} \mathrm{~J}_{\mathrm{HH}}=7.5 \mathrm{~Hz}, \mathrm{~m}-\mathrm{CH}\right)$.

${ }^{13} \mathrm{C}\left\{{ }^{1} \mathrm{H}\right\}$ NMR $\left(\mathrm{C}_{6} \mathrm{D}_{6}, 100 \mathrm{MHz}\right): 144.8\left(\mathrm{~m}, \mathrm{CF}^{2}\right), 141.2\left(\mathrm{~m}, \mathrm{CF}^{3}\right), 134.2\left(\mathrm{~s}, \mathrm{o}-\mathrm{C}_{\mathrm{Ph}}\right), 130.6$ (s, p- $\left.\mathrm{C}_{\mathrm{Ph}}\right)$, $128.6\left(\mathrm{~s}, \mathrm{~m}-\mathrm{C}_{\mathrm{Ph}}\right)$. Ipso-carbons and $\mathrm{C}=\mathrm{O}$ resonances not observed.

${ }^{19} \mathrm{~F} \mathrm{NMR}\left(\mathrm{C}_{6} \mathrm{D}_{6}, 100 \mathrm{MHz}\right):-142.4\left(\mathrm{~m}, \mathrm{CF}^{2}\right),-150.9\left(\mathrm{~m}, \mathrm{CF}^{3}\right)$

HRMS (APCL) m/z: [M + H] $]^{+}$Calcd for $\mathrm{C}_{27} \mathrm{H}_{10} \mathrm{~F}_{8} \mathrm{O}_{5}, 567.0473$; Found 567.0471. 
<smiles>O=C(Oc1c(F)c(F)c([N+](=O)[O-])c(F)c1F)c1ccccc1</smiles>

1p 4-benzoic-2,3,5,6-tetrafluoronitrobenzene: Isolation achieved by column chromatography in 95:5 $\mathrm{n}$-hexane: ethyl acetate. Isolated as a white crystalline solid. $\mathrm{R}_{\mathrm{F}}=0.43$ (164 mg, $37.3 \%, 0.52 \mathrm{mmol}$ ).

${ }^{1} \mathrm{H}$ NMR $\left(\mathrm{C}_{6} \mathrm{D}_{6}, 400 \mathrm{MHz}\right): 7.96\left(\mathrm{~d}, 2 \mathrm{H},{ }^{3} \mathrm{~J}_{\mathrm{HH}}=8.5 \mathrm{~Hz}, \mathrm{o}-\mathrm{CH}\right), 7.07\left(\mathrm{t}, 1 \mathrm{H},{ }^{3} \mathrm{~J}_{\mathrm{HH}}=8.0 \mathrm{~Hz}, \mathrm{p}-\mathrm{CH}\right), 6.94$ $\left(\mathrm{t}, 2 \mathrm{H},{ }^{3} \mathrm{~J}_{\mathrm{HH}}=8.1 \mathrm{~Hz}, \mathrm{~m}-\mathrm{CH}\right)$.

${ }^{13} \mathrm{C}\left\{{ }^{1} \mathrm{H}\right\}$ NMR ( $\left.\mathrm{C}_{6} \mathrm{D}_{6}, 100 \mathrm{MHz}\right): 161.4(\mathrm{~s}, \underline{\mathrm{C} O P h}), 142.1\left(\mathrm{~m}, \mathrm{CF}^{2}\right), 139.5\left(\mathrm{~m}, \mathrm{CF}^{3}\right), 134.6\left(\mathrm{~s}, \mathrm{o}-\mathrm{C}_{\mathrm{Ph}}\right)$, $130.5\left(\mathrm{~m}, \mathrm{C}^{4}-\mathrm{O}\right.$ ), 128.7 (s, 1C, p- $\left.\mathrm{C}_{\mathrm{Ph}}\right), 128.6$ (s, m- $\left.\mathrm{C}_{\mathrm{Ph}}\right), 126.3$ (s, i-C $\left.\mathrm{C}_{\mathrm{Ph}}\right)$. Ipso carbon $\mathrm{C}^{1}-\mathrm{NO}_{2}$ not observed.

${ }^{19} \mathrm{~F}$ NMR $\left(\mathrm{C}_{6} \mathrm{D}_{6}, 100 \mathrm{MHz}\right):-146.9\left(\mathrm{~m}, \mathrm{CF}^{2}\right),-149.9\left(\mathrm{~m}, \mathrm{CF}^{3}\right)$

IR ( $\left.\mathrm{cm}^{-1}\right)$ : 1767 ( $\mathrm{C}=\mathrm{O}$ str), 1551 ( $\mathrm{N}=\mathrm{O}$ assym.str), 1357 ( $\mathrm{N}=\mathrm{O}$ sym.str), 1234 (C-O str), 1107 (CO str).

HRMS (APCL) m/z: [M+ H] ${ }^{+}$Calcd for $\mathrm{C}_{13} \mathrm{H}_{6} \mathrm{~F}_{4} \mathrm{NO}_{4}$ 316.0227; Found 316.0228. 
<smiles>O=C(OC(=O)c1ccccc1)Oc1c(F)c(F)c([N+](=O)[O-])c(F)c1F</smiles>

1p' 2,4-dibenzoic-3,5,6-trifluorobenzonitrile: Isolation achieved by column chromatography in 95:5 hexane: ethyl acetate. Isolated as a white crystalline solid. $R_{F}=0.26$. $(87.8 \mathrm{mg}, 36.2 \%$, $0.21 \mathrm{mmol})$.

${ }^{1} \mathrm{H} \mathrm{NMR}\left(\mathrm{C}_{6} \mathrm{D}_{6}, 400 \mathrm{MHz}\right): 7.95\left(\mathrm{~d}, 4 \mathrm{H},{ }^{3} \mathrm{~J}_{\mathrm{HH}}=7.8 \mathrm{~Hz}, \mathrm{o}-\mathrm{CH}\right), 7.08\left(\mathrm{t}, 2 \mathrm{H},{ }^{3} \mathrm{~J}_{\mathrm{HH}}=7.4 \mathrm{~Hz}, \mathrm{p}-\mathrm{CH}\right)$, $6.95\left(\mathrm{t}, 4 \mathrm{H},{ }^{3} \mathrm{~J}_{\mathrm{HH}}=7.7 \mathrm{~Hz}, \mathrm{~m}-\mathrm{CH}\right)$.

${ }^{13} \mathrm{C}\left\{{ }^{1} \mathrm{H}\right\}$ NMR ( $\left.\mathrm{C}_{6} \mathrm{D}_{6}, 100 \mathrm{MHz}\right): 162.3$ (s, 4-OㄷO 161.7 (s, 2-OㅡoPh), $145.5\left(\mathrm{~m}, \mathrm{CF}^{6}\right), 142.9$ $\left(\mathrm{m}, \mathrm{CF}^{5}\right), 141.7\left(\mathrm{~m}, \mathrm{CF}^{3}\right), 134.4\left(\mathrm{~d}, \mathrm{~J}_{\mathrm{CF}}=11.2 \mathrm{~Hz}, \mathrm{o}-\mathrm{C}_{\mathrm{Ph}}\right), 133.8$ (s, o- $\left.\mathrm{C}_{\mathrm{Ph}}\right), 130.6$ (s, p- $\left.\mathrm{C}_{\mathrm{Ph}}\right), 130.3$ $\left(\mathrm{s}, \mathrm{p}-\mathrm{C}_{\mathrm{ph}}\right), 128.6$ (s, m- $\left.\mathrm{C}_{\mathrm{ph}}\right), 128.5$ (s, m- $\left.\mathrm{C}_{\mathrm{ph}}\right), 126.6$ (s, i- $\left.\mathrm{C}_{\mathrm{ph}}\right), 126.5$ (s, i- $\mathrm{C}_{\mathrm{ph}}$ ). Ipso carbons of fluoroarene moiety not observed.

${ }^{19} \mathrm{~F} N M R\left(C_{6} D_{6}, 100 \mathrm{MHz}\right):-139.7\left(\mathrm{~d},{ }^{3} \mathrm{~J}_{\mathrm{FF}}=8.3 \mathrm{~Hz}, \mathrm{CF}^{6}\right),-146.1\left(\mathrm{~d},{ }^{3} \mathrm{~J}_{\mathrm{FF}}=23.7 \mathrm{~Hz}, \mathrm{CF}^{5}\right),-146.5(\mathrm{~m}$, $\left(F^{3}\right)$.

IR (cm $\left.{ }^{-1}\right): 1759$ (C=O str), 1715 (C=O str), 1551 ( $\mathrm{N}=\mathrm{O}$ asym.str), 1357 ( $\mathrm{N}=\mathrm{O}$ sym.str), 1208 (C-O str), 1170 (C-O str), 1103 (C-O str).

HRMS (APCL) m/z: [M + H] ${ }^{+}$Calcd for $\mathrm{C}_{20} \mathrm{H}_{10} \mathrm{~F}_{3} \mathrm{NO}_{6} \mathrm{Na}$ 440.0352; Found 440.0357. 
<smiles>N#Cc1c(F)c(F)c(OC(=O)c2ccccc2)c(F)c1F</smiles>

1q, 4-benzoic-2,3,5,6-tetrafluorobenzonitrile: Isolation achieved by column chromatography in 9: $1 \mathrm{n}$-pentane: ethyl acetate. Isolated as a white crystalline solid. $\mathrm{R}_{\mathrm{F}}=0.55$. $(131 \mathrm{mg}, 31.5$ $\%, 0.44 \mathrm{mmol}){ }^{[\mathrm{s} 8]}$

${ }^{1} \mathrm{H} \mathrm{NMR}\left(\mathrm{C}_{6} \mathrm{D}_{6}, 400 \mathrm{MHz}\right): 7.97\left(\mathrm{~d}, 2 \mathrm{H},{ }^{3} \mathrm{~J}_{\mathrm{HH}}=7.6 \mathrm{~Hz}, \mathrm{o}-\mathrm{CH}\right), 7.13\left(\mathrm{t}, 1 \mathrm{H},{ }^{3} \mathrm{~J}_{\mathrm{HH}}=7.6 \mathrm{~Hz}, \mathrm{p}-\mathrm{CH}\right), 7.01$ $\left(\mathrm{t}, 2 \mathrm{H}, 3^{3} \mathrm{JH}_{\mathrm{HH}}=8.5 \mathrm{~Hz}, \mathrm{~m}-\mathrm{CH}\right)$.

${ }^{13} \mathrm{C}\left\{{ }^{1} \mathrm{H}\right\}$ NMR $\left(\mathrm{C}_{6} \mathrm{D}_{6}, 100 \mathrm{MHz}\right): 161.5$ (s, COPh), $147.5\left(\mathrm{~m}, \mathrm{CF}^{2}\right), 141.0\left(\mathrm{~m}, \mathrm{CF}^{3}\right), 134.5$ (s, o- $\left.\mathrm{C}_{\mathrm{Ph}}\right)$, 130.5 (s, p- $\left.C_{P h}\right), 128.7$ (s, m- $\left.C_{P h}\right) 126.3$ (s, 1C, i- $C_{P h}$ ). Ipso carbons of fluoroarene not observed, nitrile carbon not observed.

${ }^{19} \mathrm{~F}$ NMR $\left(\mathrm{C}_{6} \mathrm{D}_{6}, 100 \mathrm{MHz}\right):-133.5\left(\mathrm{~m}, \mathrm{CF}^{2}\right),-150.3\left(\mathrm{~m}, \mathrm{CF}^{3}\right)$.

IR ( $\left.\mathrm{cm}^{-1}\right): 2231$ (CN str), 1767 (C=O str), 1233 (C-O str), 1118 (C-O str).

HRMS (APCL) m/z: [M + H] $]^{+}$Calcd for $\mathrm{C}_{14} \mathrm{H}_{5} \mathrm{~F}_{4} \mathrm{NO}_{2}$ 296.0329; Found 296.0332. 
<smiles>N#Cc1c(F)c(F)c(OC(=O)c2ccccc2)c(F)c1OC(=O)c1ccccc1</smiles>

1q', 2,4-dibenzoic-3,5,6-trifluorobenzonitrile: Isolation achieved by column chromatography in 9:1 n-pentane: ethyl acetate. Isolated as a white crystalline solid. $R_{F}=0.30$ (199 mg, 35.9 $\%, 0.50 \mathrm{mmol}$ ). Recrystallized for XRD characterisation by diffusion of $n$-pentane into ethyl acetate solution at room temperature.

${ }^{1} \mathrm{H} N M R\left(C_{6} D_{6}, 400 \mathrm{MHz}\right): 7.95\left(\mathrm{~d}, 4 \mathrm{H},{ }^{3} \mathrm{~J}_{\mathrm{HH}}=7.9 \mathrm{~Hz}, \mathrm{o}-\mathrm{CH}\right), 7.08\left(\mathrm{t}, 2 \mathrm{H},{ }^{3} \mathrm{~J}_{\mathrm{HH}}=7.2 \mathrm{~Hz}, \mathrm{p}-\mathrm{CH}\right), 6.95$ $\left(t, 4 \mathrm{H},{ }^{3} \mathrm{~J}_{\mathrm{HH}}=7.7 \mathrm{~Hz}, \mathrm{~m}-\mathrm{CH}\right)$.

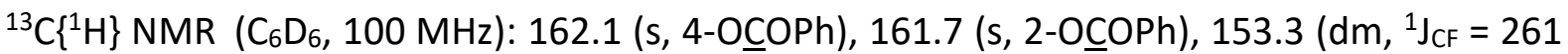
$\left.\mathrm{Hz}, \mathrm{CF}^{6}\right), 148.6\left(\mathrm{dm},{ }^{1} \mathrm{~J}_{\mathrm{CF}}=260 \mathrm{~Hz}, \mathrm{CF}^{5}\right), 145.8\left(\mathrm{dm},{ }^{1} \mathrm{~J}_{\mathrm{CF}}=263 \mathrm{~Hz}, \mathrm{CF}^{3}\right), 134.3\left(\mathrm{~s}, \mathrm{o}-\mathrm{C}_{\mathrm{Ph}}\right), 133.8$ (s, o-C $\left.C_{p h}\right), 130.6,\left(s, p-C_{p h}\right), 130.4$ (s, p-C $\left.C_{p h}\right), 128.7$ (s, m-Cph), 128.5 (s, m-Cph), 126.7 (s, i-Cph), $126.5\left(\mathrm{~s}, \mathrm{i}-\mathrm{C}_{\mathrm{Ph}}\right) 108.1$ (s, $\left.\underline{\mathrm{CN}}\right)$. Ipso carbons of fluoroarene not observed.

${ }^{19} \mathrm{~F}$ NMR $\left(\mathrm{C}_{6} \mathrm{D}_{6}, 100 \mathrm{MHz}\right):-132.5\left(\mathrm{~m}, \mathrm{CF}^{3}\right),-139.4\left(\mathrm{~d},{ }^{3} \mathrm{~J}_{\mathrm{FF}}=11.0 \mathrm{~Hz}, \mathrm{CF}^{5}\right),-147.0\left(\mathrm{~d},{ }^{3} \mathrm{~J}_{\mathrm{FF}}=21.1\right.$ $\left.\mathrm{Hz}, \mathrm{CF}^{6}\right)$.

IR ( $\mathrm{cm}^{-1}$ ): 2170 (CN str), 1774 (C=O str), 1715 (C=O str), 1207 (C-O str), 1170 (C-O str), 1036 (CO str).

HRMS (APCL) m/z: [M + H] $]^{+}$Calcd for $\mathrm{C}_{21} \mathrm{H}_{11} \mathrm{~F}_{3} \mathrm{NO}_{4}$ 398.0635; Found 398.0631. 
<smiles>O=C(F)c1ccccc1</smiles>

2a Benzoyl fluoride: Isolation achieved by column chromatography in 7:3 hexane: dichloromethane. Isolated as a colourless oil. $\mathrm{R}_{\mathrm{F}}=0.72 .(126.1 \mathrm{mg}, 72.6 \%, 1.02 \mathrm{mmol})$. ${ }^{[59]}$

${ }^{1} \mathrm{H} \mathrm{NMR}\left(\mathrm{C}_{6} \mathrm{D}_{6}, 400 \mathrm{MHz}\right): 7.67\left(\mathrm{~d}, 2 \mathrm{H},{ }^{3} \mathrm{~J}_{\mathrm{HH}}=7.7 \mathrm{~Hz}, \mathrm{o}-\mathrm{CH}\right), 6.99\left(\mathrm{t}, 1 \mathrm{H},{ }^{3} \mathrm{~J}_{\mathrm{HH}}=7.5 \mathrm{~Hz}, \mathrm{p}-\mathrm{CH}\right), 6.82$ (t, $\left.2 \mathrm{H},{ }^{3} \mathrm{~J}_{\mathrm{HH}}=8.6 \mathrm{~Hz}, \mathrm{~m}-\mathrm{CH}\right)$.

${ }^{13} \mathrm{C}\left\{{ }^{1} \mathrm{H}\right\} \operatorname{NMR}\left(\mathrm{C}_{6} \mathrm{D}_{6}, 100 \mathrm{MHz}\right): 158.7\left(\mathrm{~d}, 1 \mathrm{C},{ }^{1} \mathrm{~J}_{\mathrm{CF}}=342 \mathrm{~Hz}, \underline{\mathrm{COF}}\right), 134.5$ (s, o- $\left.\mathrm{C}_{\mathrm{Ph}}\right), 131.0$ (s, p$\left.\mathrm{C}_{\mathrm{Ph}}\right), 128.5\left(\mathrm{~s}, \mathrm{~m}-\mathrm{C}_{\mathrm{Ph}}\right)$. Ipso carbon not observed.

${ }^{19} \mathrm{~F}$ NMR $\left(\mathrm{C}_{6} \mathrm{D}_{6}, 100 \mathrm{MHz}\right): 17.9(\mathrm{~s})$.<smiles>CC(=O)F</smiles>

2b Acetyl fluoride: Product not isolated, NMR yield $=99 \pm 4 \%$ based on ${ }^{19} \mathrm{~F}$ NMR in reaction $1 b$.

${ }^{19} \mathrm{~F}$ NMR $\left(\mathrm{C}_{6} \mathrm{D}_{6}, 100 \mathrm{MHz}\right): 50.5(\mathrm{~s})$ in close agreement with the current literature. ${ }^{[\mathrm{S} 10]}$<smiles>CC=CC(=O)F</smiles>

2c Crotonyl fluoride: Product not isolated, NMR yield $=99 \pm 1 \%$ based on ${ }^{19} \mathrm{~F}$ NMR.

${ }^{19} \mathrm{~F} \mathrm{NMR}\left(\mathrm{C}_{6} \mathrm{D}_{6}, 100 \mathrm{MHz}\right): 23.0(\mathrm{~s})$<smiles>CCC(C)C(C)=O</smiles>

2d Decanoyl fluoride: Product not isolated, NMR yield $=66 \pm 2 \%$ based on ${ }^{19} \mathrm{~F} \mathrm{NMR}$. ${ }^{19} \mathrm{~F}$ NMR $\left(\mathrm{C}_{6} \mathrm{D}_{6}, 100 \mathrm{MHz}\right): 43.4(\mathrm{~s})$ in close agreement with the current literature. ${ }^{[\mathrm{S} 11]}$<smiles>COC(=O)F</smiles>

2e Methyl carbonofluoridate: Product not isolated, NMR yield $=39 \%$ based on ${ }^{19} \mathrm{~F} \mathrm{NMR}$.

${ }^{19} \mathrm{~F} \mathrm{NMR}\left(\mathrm{C}_{6} \mathrm{D}_{6}, 100 \mathrm{MHz}\right):-20.0$ (s) in close agreement with the current literature. ${ }^{[\mathrm{S} 12]}$ 


\section{X-ray Crystal Structures.}

\section{The X-ray crystal structure of $1 \mathbf{k}$}

Crystal data for $\mathbf{1 k}: \mathrm{C}_{15} \mathrm{H}_{8} \mathrm{~F}_{4} \mathrm{O}_{3}, M=312.21$, monoclinic, $C 2 / c$ (no. 15), $a=25.3257(12), b=$ 5.9112(3), $c=17.3301(9) \AA, \beta=97.384(5)^{\circ}, V=2572.9(2) \AA^{3}, Z=8, D_{c}=1.612 \mathrm{~g} \mathrm{~cm}^{-3}, \mu$ (Mo$\mathrm{K} \alpha)=0.150 \mathrm{~mm}^{-1}, T=173 \mathrm{~K}$, colourless tablets, Agilent Xcalibur $3 \mathrm{E}$ diffractometer; 2587 independent measured reflections $\left(R_{\text {int }}=0.0159\right), F^{2}$ refinement, ${ }^{[\mathrm{S} 13][\mathrm{S} 14]} R_{1}(\mathrm{obs})=0.0366$, $w R_{2}$ (all) $=0.0938,2117$ independent observed absorption-corrected reflections $\left[\left|F_{0}\right|>\right.$ $4 \sigma\left(\left|F_{\mathrm{o}}\right|\right)$, completeness to $\left.\theta_{\text {full }}\left(25.2^{\circ}\right)=98.6 \%\right], 201$ parameters. CCDC 2021812.

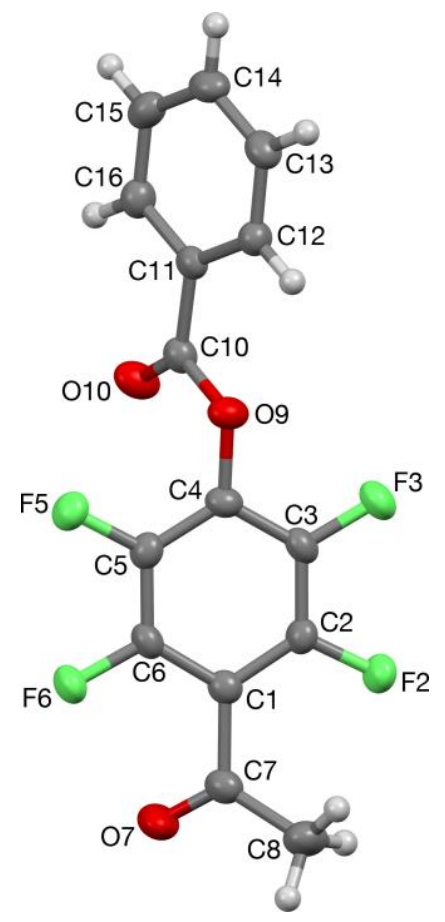

Figure S1. ORTEP diagram showing the crystal structure of $\mathbf{1 k}$ ( $50 \%$ probability ellipsoids). 


\section{The X-ray crystal structure of $1 \mathbf{q}^{\prime}$}

Crystal data for 1q': $\mathrm{C}_{21} \mathrm{H}_{10} \mathrm{~F}_{3} \mathrm{NO}_{4}, M=397.30$, monoclinic, $P 2_{1} / c$ (no. 14 ), $a=17.0077(5), b=$ 7.5346(2), $c=13.5271(4) \AA, \beta=92.463(3)^{\circ}, V=1731.84(9) \AA^{3}, Z=4, D_{c}=1.524 \mathrm{~g} \mathrm{~cm}^{-3}, \mu($ Mo$\mathrm{K} \alpha)=0.127 \mathrm{~mm}^{-1}, T=173 \mathrm{~K}$, colourless blocky needles, Agilent Xcalibur $3 \mathrm{E}$ diffractometer; 3896 independent measured reflections $\left(R_{\text {int }}=0.0354\right), F^{2}$ refinement, ${ }^{[\mathrm{S} 13],[\mathrm{S} 14]} R_{1}(\mathrm{obs})=$ $0.0452, w R_{2}($ all $)=0.1089,2598$ independent observed absorption-corrected reflections $\left[\left|F_{0}\right|\right.$ $>4 \sigma\left(\left|F_{\mathrm{o}}\right|\right)$, completeness to $\theta_{\text {full }}\left(25.2^{\circ}\right)=100 \%$ ], 262 parameters. CCDC 2021813.

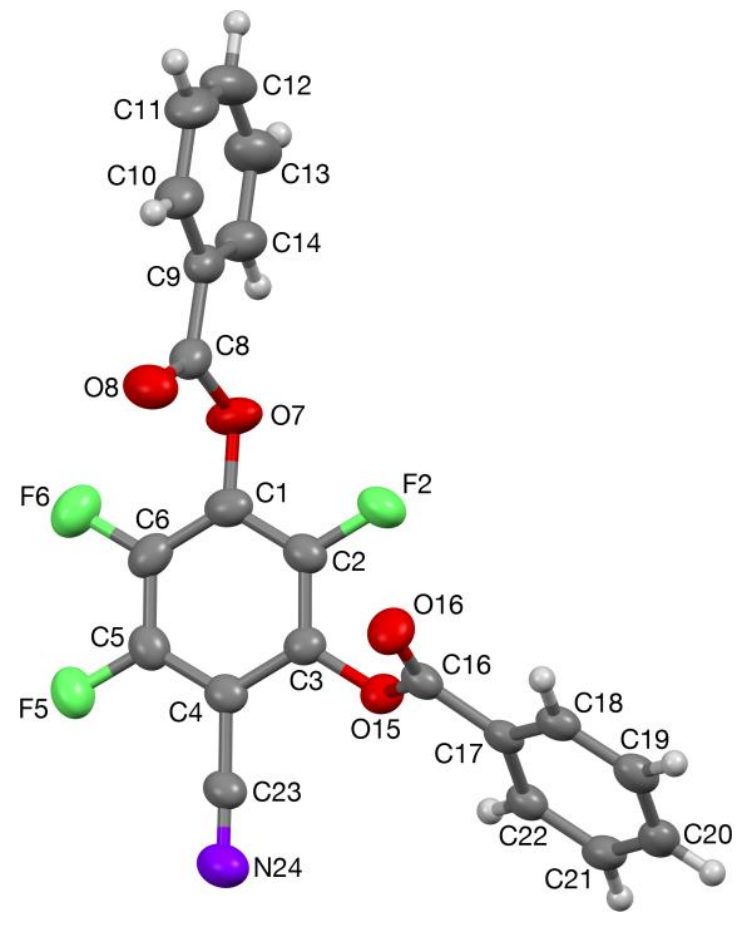

Figure S2. ORTEP diagram showing the crystal structure of 1q' (50\% probability ellipsoids). 


\section{DFT studies}

\subsection{Dual Catalysed Pathway}

DFT calculations were run using Gaussian 09 (Revision D.01) using the B3LYP, B3PW91 and $\omega B 97 X-D$ density functionals. The $6-31 G^{* *}$ basis set was used for all atoms. Geometry optimisation calculations were performed without symmetry constraints. Frequency analyses for all stationary points were performed to confirm the nature of the structures as either minima (no imaginary frequency) or transition states (only one imaginary frequency). Intrinsic reaction coordinate (IRC) calculations followed by full geometry optimisations on final points were used to connect transition states and minima located on the potential energy surface allowing a full energy profile (calculated at $298.15 \mathrm{~K}, 1 \mathrm{~atm}$ ) of the reaction to be constructed. Free energies reported are corrected for the effects of acetonitrile $(\varepsilon=36.64)$ solvent using the polarizable continuum model (PCM). Calculations run using the B3LYP or B3PW91 functionals were corrected with GD3-BJ empirical dispersion inclusion. The graphical user interface used to visualise the various properties of the intermediates and transition states was GaussView 5.0.9.

(a)
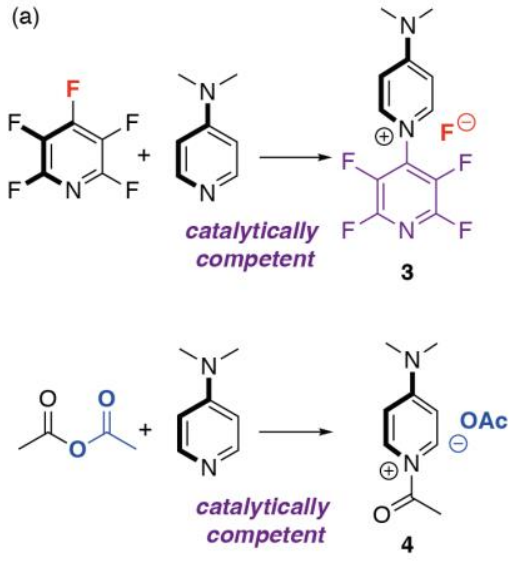

(b)

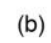

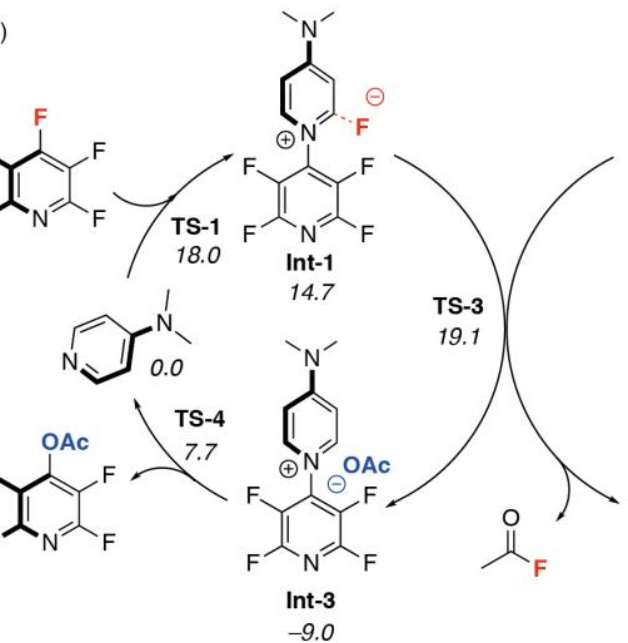

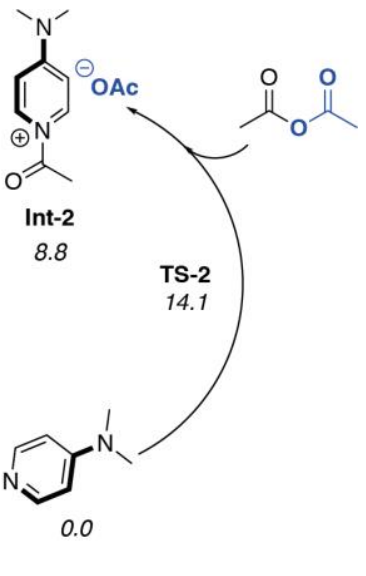

\begin{tabular}{llll} 
& B3LYP $\left(\mathrm{Kcal} \mathrm{mol}^{-1}\right)$ & B3PW91 $\left(\mathrm{Kcal} \mathrm{mol}^{-1}\right)$ & WB97X-D $\left(\mathrm{Kcal} \mathrm{mol}^{-1}\right)$ \\
\hline TS-1 & 18.0 & 17.9 & 21.6 \\
Int-1 & 14.7 & 14.3 & 15.8 \\
TS-2 & 14.1 & 13.6 & 16.5 \\
Int-2 & 8.8 & 9.7 & 11.6 \\
TS-3 & 19.1 & 25.1 & 27.6 \\
Int-3 & -9.0 & -9.2 & -9.5 \\
TS-4 & 7.7 & 8.7 & 9.5 \\
Product & -5.8 & -6.3 & -6.0
\end{tabular}

Table S3. Functional testing for the energy profile of the dual-catalysed reaction, shown in Figure 2. The 6-31G** basis set was used. Empirical dispersion ( $g d 3 b j$ ) and solvent interactions ( $\mathrm{scrf}=\mathrm{pcm}$, acetonitrile) were included in calculations. 


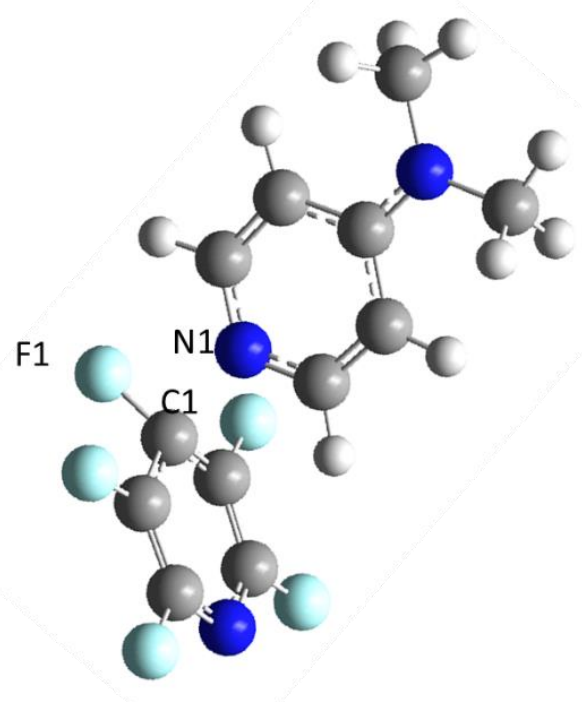

TS-1: N1-C1 = 1.85. C1-F1 = 1.38

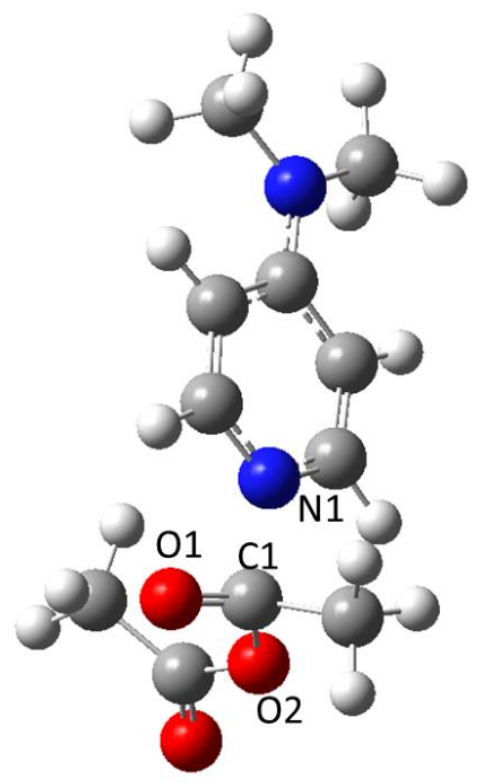

TS-2: $\mathrm{N} 1-\mathrm{C} 1=1.78 . \mathrm{C} 1-\mathrm{O} 2=1.50$.

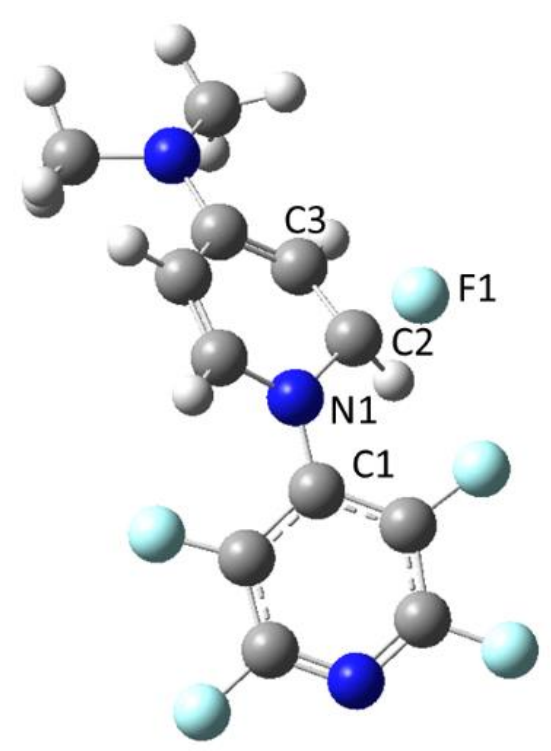

Int-1: N1-C1 = 1.39. C2-F1 = 1.43. C1-N1-C2-C3 dihedral $=146.7$

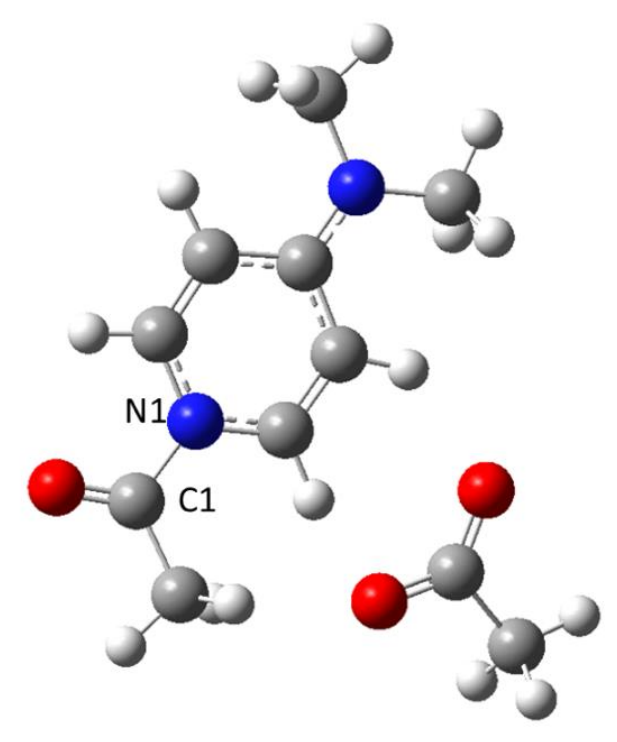

Int-2: N1-C1 = 1.45 . 


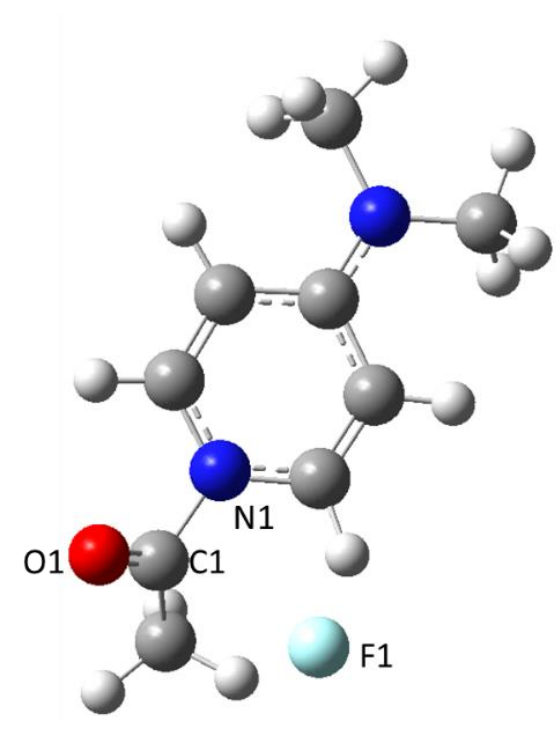

TS-3: C1---F1 = 2.54. C1-N1 = 1.49.

Tetrafluoropyridinium salt omitted for clarity.

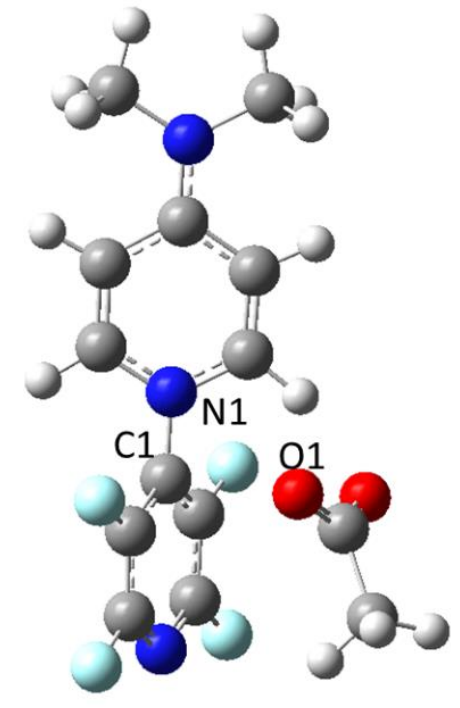

Int-3: $\mathrm{C} 1-\mathrm{N} 1=1.42 . \mathrm{C} 1---\mathrm{O} 1=2.93$.

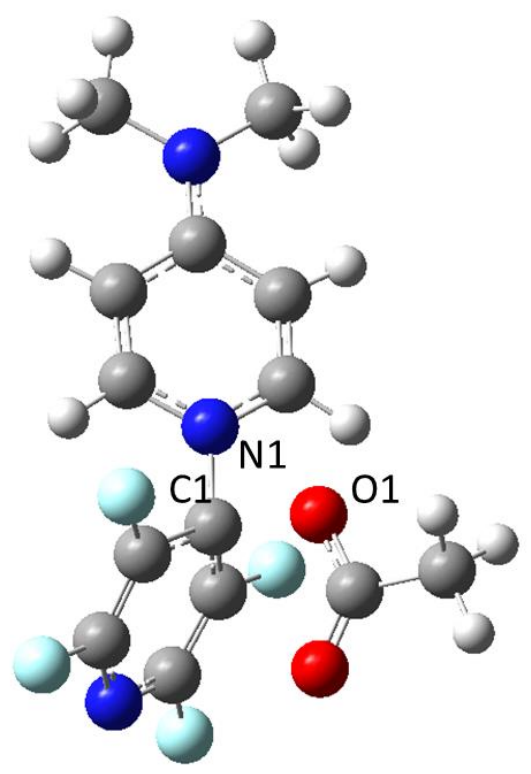

TS-4: N1-C1 = 1.49. C1---O1 = 1.73.

Table S4. Calculated structures of transition states (TS) and intermediates (Int) using B3LYP ((GD3BJ), PCM(acetonitrile)//6-31GG(d,p). Selected bond distances $(\AA)$ and angles $\left({ }^{\circ}\right)$ are reported. 


\subsection{Mono-Catalysed Pathway}

In addition to the dual catalysed reaction pathway presented in Figure 2, the individual monocatalysed reaction pathways were also considered in Figure S4. For each functional, the overall activation barrier was lower for the dual catalysed pathway than either monocatalysed pathway. This finding is consistent with kinetic data supporting the conclusion that the turnover-limiting step, and therefore overall reaction pathway, involves two molecules of DMAP.

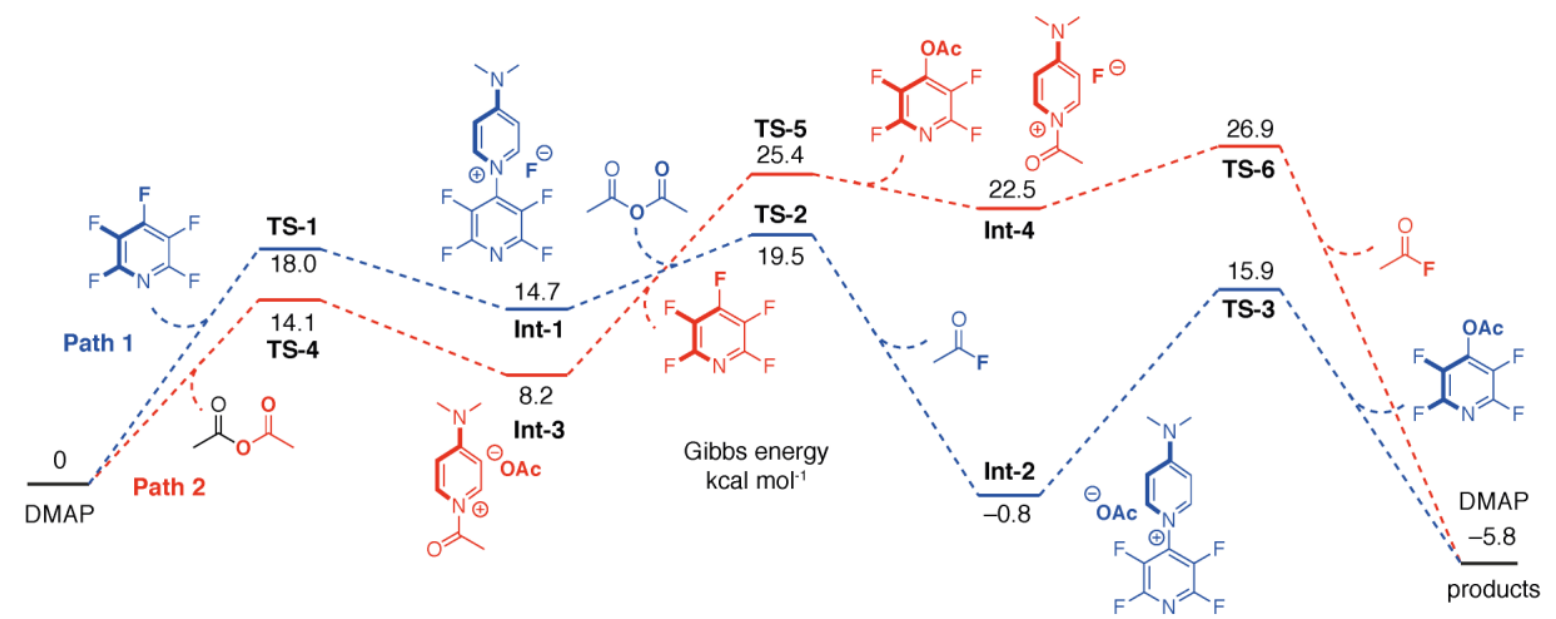

Figure S4. DFT calculated reaction pathways for the mono-catalysed reaction of DMAP, C5F4N and acetic anhydride.

\begin{tabular}{llll} 
& B3LYP $\left(\mathrm{Kcal} \mathrm{mol}^{-\mathbf{1}}\right)$ & B3PW91 $\left(\mathrm{Kcal} \mathrm{mol}^{-\mathbf{1}}\right)$ & WB97X-D $\left(\mathrm{Kcal} \mathrm{mol}^{-\mathbf{1}}\right)$ \\
\hline TS-1 & 18.0 & 17.9 & 21.6 \\
Int-1 & 14.7 & 14.3 & 15.8 \\
TS-2 & 19.5 & 20.3 & 21.3 \\
Int-2 & -0.8 & 0.4 & 2.08 \\
TS-3 & 15.9 & 18.3 & 21.1 \\
TS-4 & 14.1 & 13.6 & 16.5 \\
Int-3 & 8.2 & 9.7 & 11.6 \\
TS-5 & 25.4 & 25.3 & 31.4 \\
Int-4 & 22.5 & 22.9 & 26.0 \\
TS-6 & 26.9 & 28.1 & 31.1 \\
Product & -5.8 & -6.3 & -6.0
\end{tabular}

Table S5. Functional testing for the energy profile of the reaction pathways 1 and 2, shown in Figure 2. The 6$31 \mathrm{G}^{* *}$ basis set was used. Empirical dispersion ( $\mathrm{gd} 3 \mathrm{bj}$ ) and solvent interactions (scrf=pcm, acetonitrile) were included. 
In Figure S5, the differences in transition state barriers for key steps of the mono and dual catalysed processes are compared.

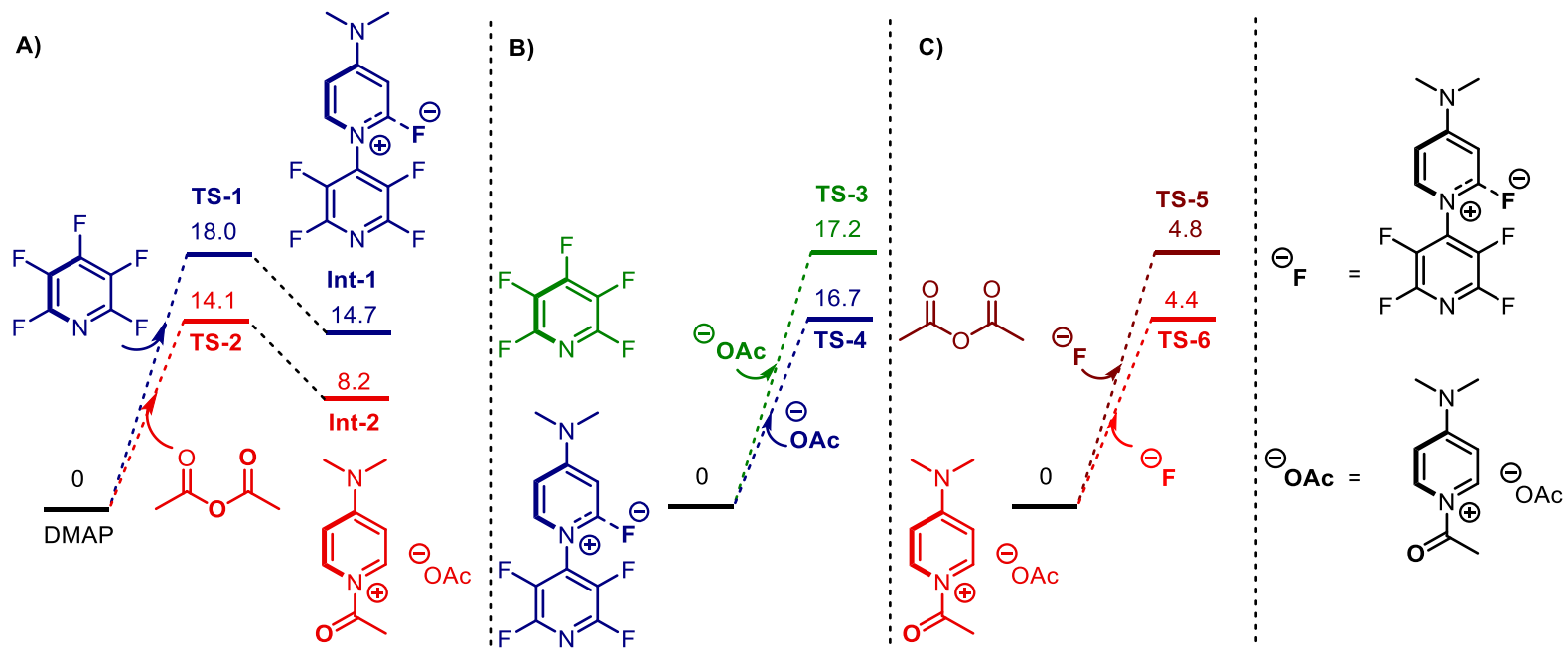

Figure S5. Comparisons of transition state barriers for the mono and dual catalysed reaction pathways. The B3LYP functional and 6-31G** basis set were used. Empirical dispersion (gd3bj) and solvent interactions (scrf $=\mathrm{pcm}$, acetonitrile) were included. 


\section{Kinetics}

Reactions were monitored in situ in a Bruker $500 \mathrm{MHz}$ machine. ${ }^{19} \mathrm{~F}$ NMR was conducted with a scan range of 100 to $-300 \mathrm{ppm}$. Scans were performed with a 55 s delay. An FID was collected every 5 minutes. The reactions were conducted at $80^{\circ} \mathrm{C}$ to avoid potential bumping of solvent. Reactions were monitored for approximately 16 hours. Conversions were calculated from reagent and product concentrations, based on integration value, relative to an internal standard (1,4-difluorobenzene).

Kinetics were initially conducted with acetic anhydride instead of benzoic anhydride, however lower production of acyl fluoride product was observed, owing to the high volatility of this product resulting in a likely partitioning of material between solution and the headspace of the NMR tube at the temperature of the experiment.<smiles>Fc1nc(F)c(F)c(F)c1F</smiles>

[0.14 mmol]<smiles>O=C(OC(=O)c1ccccc1)c1ccccc1</smiles>

Catalyst ( 0.10 equivs) $17 \mathrm{hrs}$<smiles>O=C(F)[PH2+]c1c(F)nc(F)c(F)c1OC(=O)c1ccccc1</smiles>

[1.40 mmol]

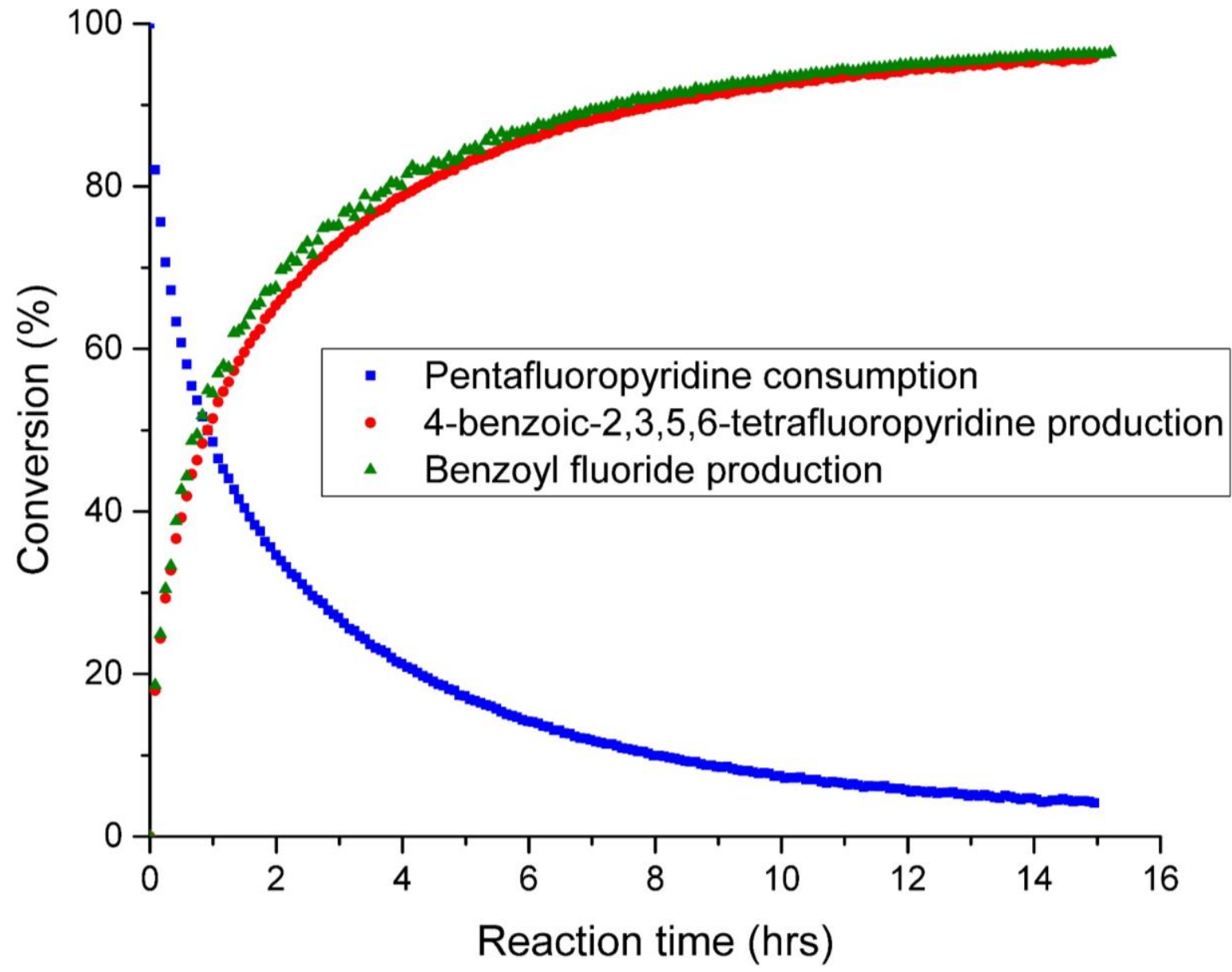

Figure S6. Plot of pentafluoropyridine consumption, 4-benzoic-2,3,5,6-tetrafluoropyridine and benzoyl fluoride production over time. 


\subsection{Pseudo First Order Reactions.}

The rate order in pentafluoropyridine and benzoic anhydride was experimentally verified through pseudo first order reactions. In both cases, when conducting the experiment in an excess of alternate reagent, a plot of the natural logarithm of rate (measured as relative concentration of pentafluoropyridine, [PFP]) against reaction time was shown to be linear, verifying the rate order of both reagents to be one<smiles>Fc1nc(F)c(F)c(F)c1F</smiles>

[0.14 $\mathrm{mmol}]$<smiles>O=C(OC(=O)c1ccccc1)Pc1ccccc1</smiles>

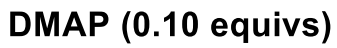

$\mathrm{CH}_{3} \mathrm{CN}, 80^{\circ} \mathrm{C}$

17 hrs<smiles>O=C(Oc1c(F)c(F)nc(F)c1F)c1ccccc1</smiles>

[1.40 $\mathrm{mmol}]$

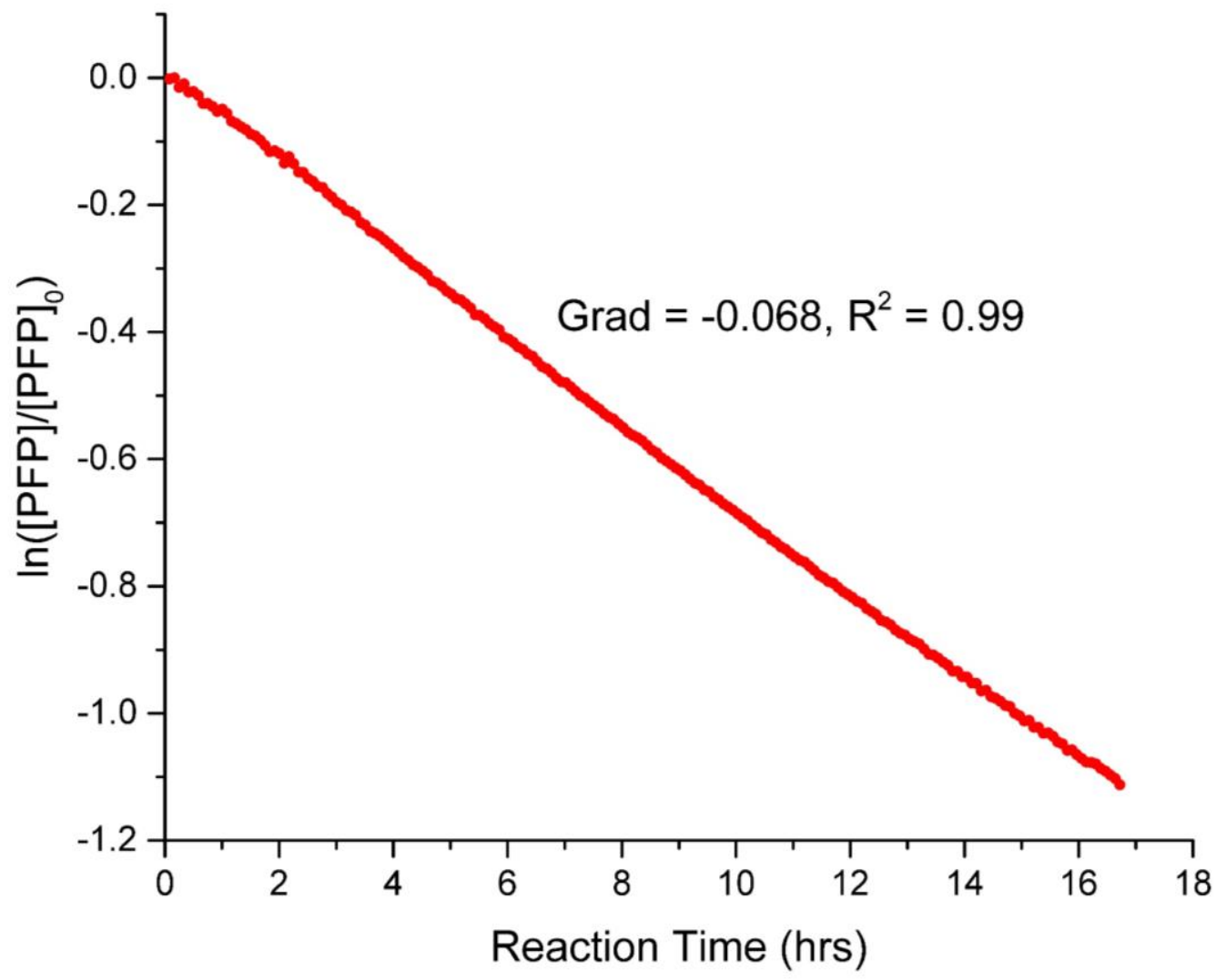

Figure S7. In plot of consumption of pentafluoropyridine over time, in a 9 equivalent excess of benzoic anhydride. 
<smiles>Fc1nc(F)c(F)c(F)c1F</smiles><smiles>C1CCCCC1</smiles><smiles>P=C(OCc1ccccc1)c1ccccc1</smiles>

[1.40 mmol]

[0.14 mmol]

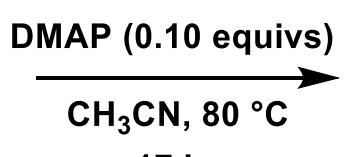

$17 \mathrm{hrs}$<smiles>O=C(F)[PH2+]c1c(F)nc(F)c(F)c1OC(=O)c1ccccc1</smiles>

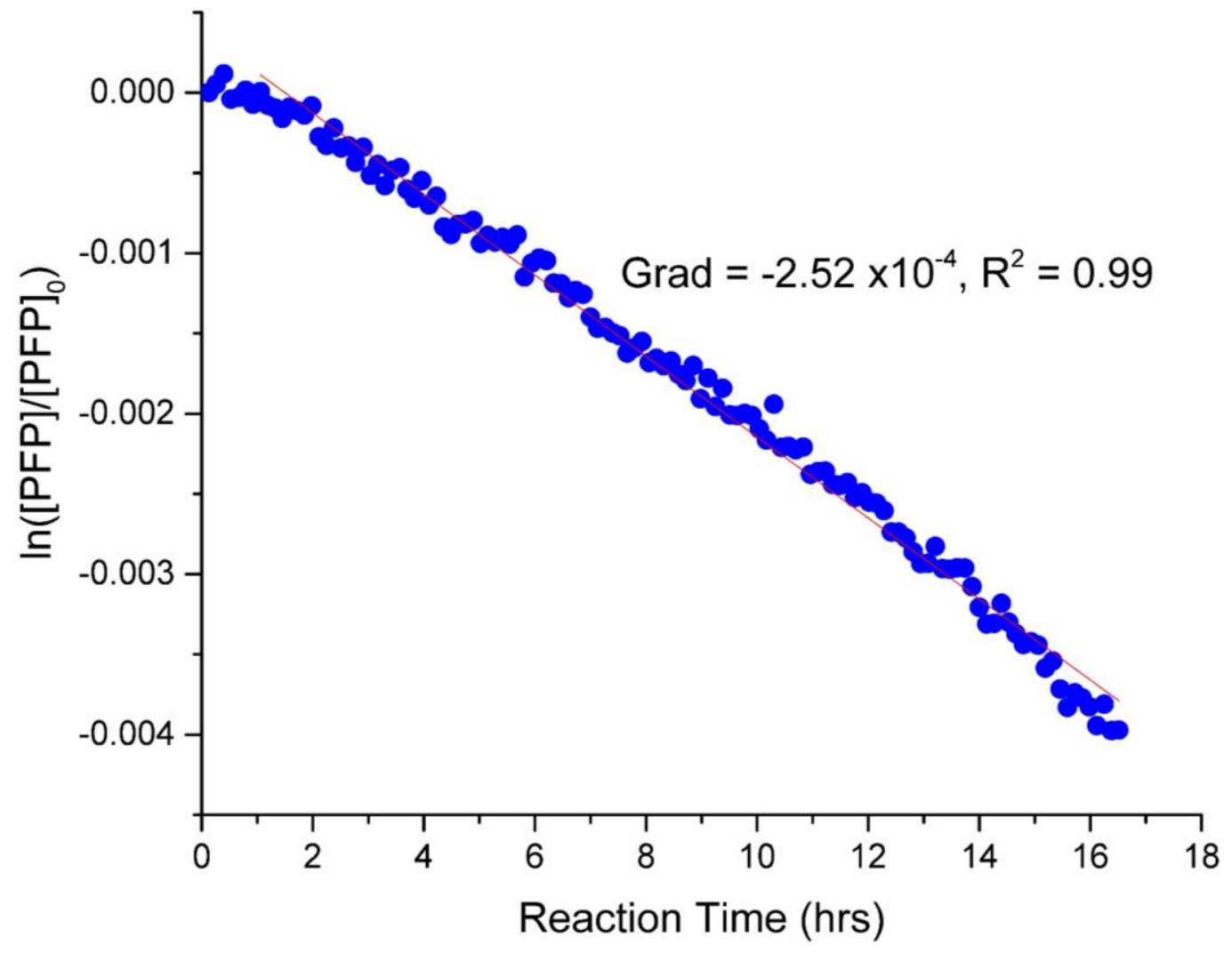

Figure 58. In plot of consumption of pentafluoropyridine over time, in a 9 equivalent excess of pentafluoropyridine to benzoic anhydride.

A very minor induction period can be observed in this reaction, it does not impact the treatment of this data or affect the overall observation that the rate of consumption of pentafluoropyridine is first order across the reaction timecourse. 


\subsection{Determining Catalyst Order.}

In order to verify the order of catalyst in the reaction, reaction progression was monitored for four catalyst loadings.

A log-log plot of initial rates against total catalyst concentration, shown in Figure S9 suggests a catalyst order of 2 . This is consistent with DMAP activation of both PFP and benzoic anhydride in the rate determining step of reaction.

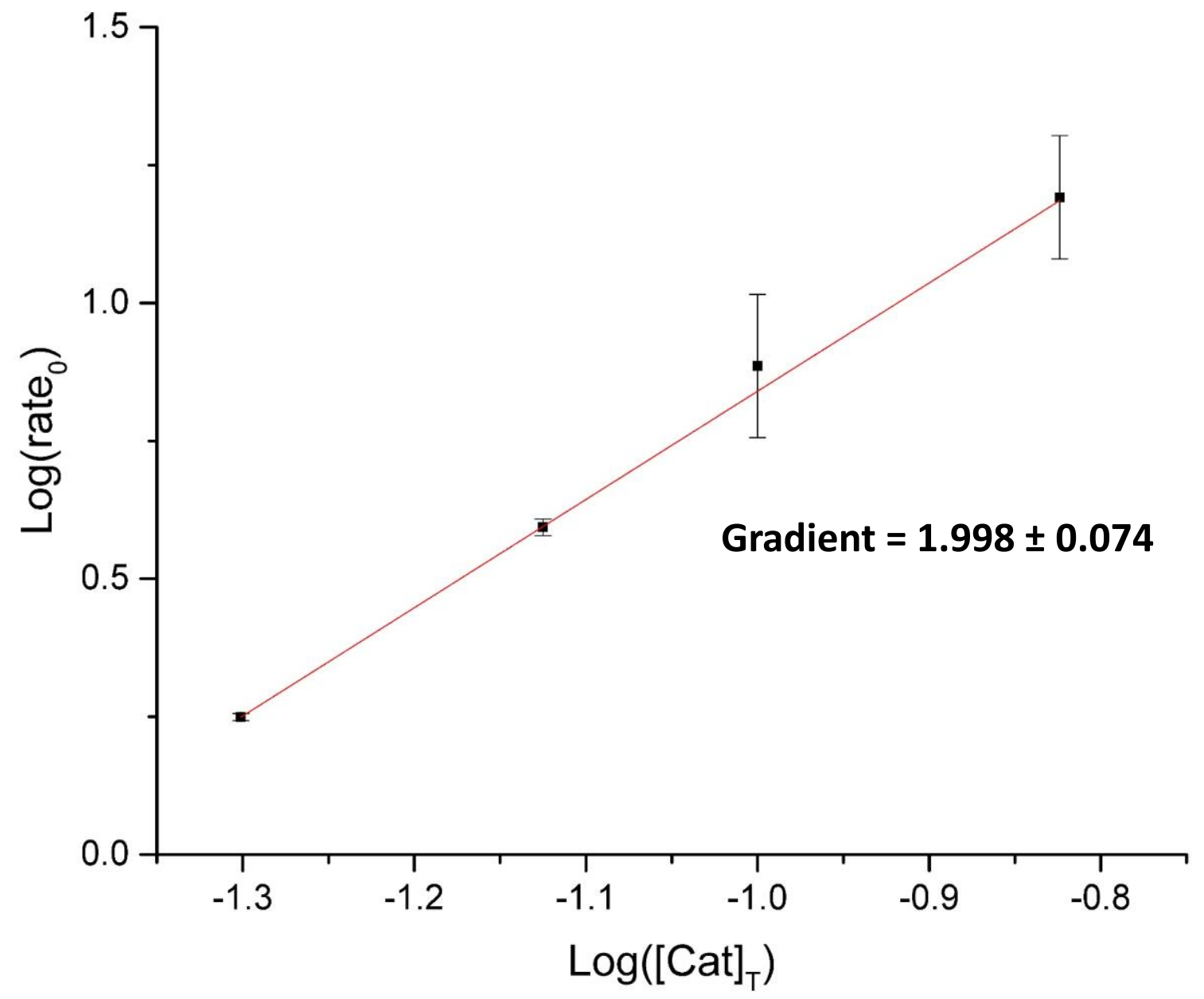

Figure S9. Log-log plot of initial rates (rateo) against total catalyst concentration ([Cat $]_{T}$ ) for four catalyst loadings: $0.05,0.075,0.10$ and 0.15 equivalents. Reactions were set up following general procedure $3 \mathrm{i}$.

In addition to initial rates, graphical analysis was applied to the entire data sets for each catalyst loading. By plotting reaction conversion as concentration of pentafluoropyridine ([PFP]) against reaction time, multiplied by catalyst concentration, to the power of an arbitrary value $\mathrm{n}\left(\mathrm{t}[\mathrm{Cat}]^{\mathrm{n}}\right)$, the order of catalyst in the reaction can be visually verified as the value of $\mathrm{n}$ for which the plots best overlap. As can be seen in Figure S10, this is consistent with a catalyst order of 2 , in agreement with the initial rates plot. 

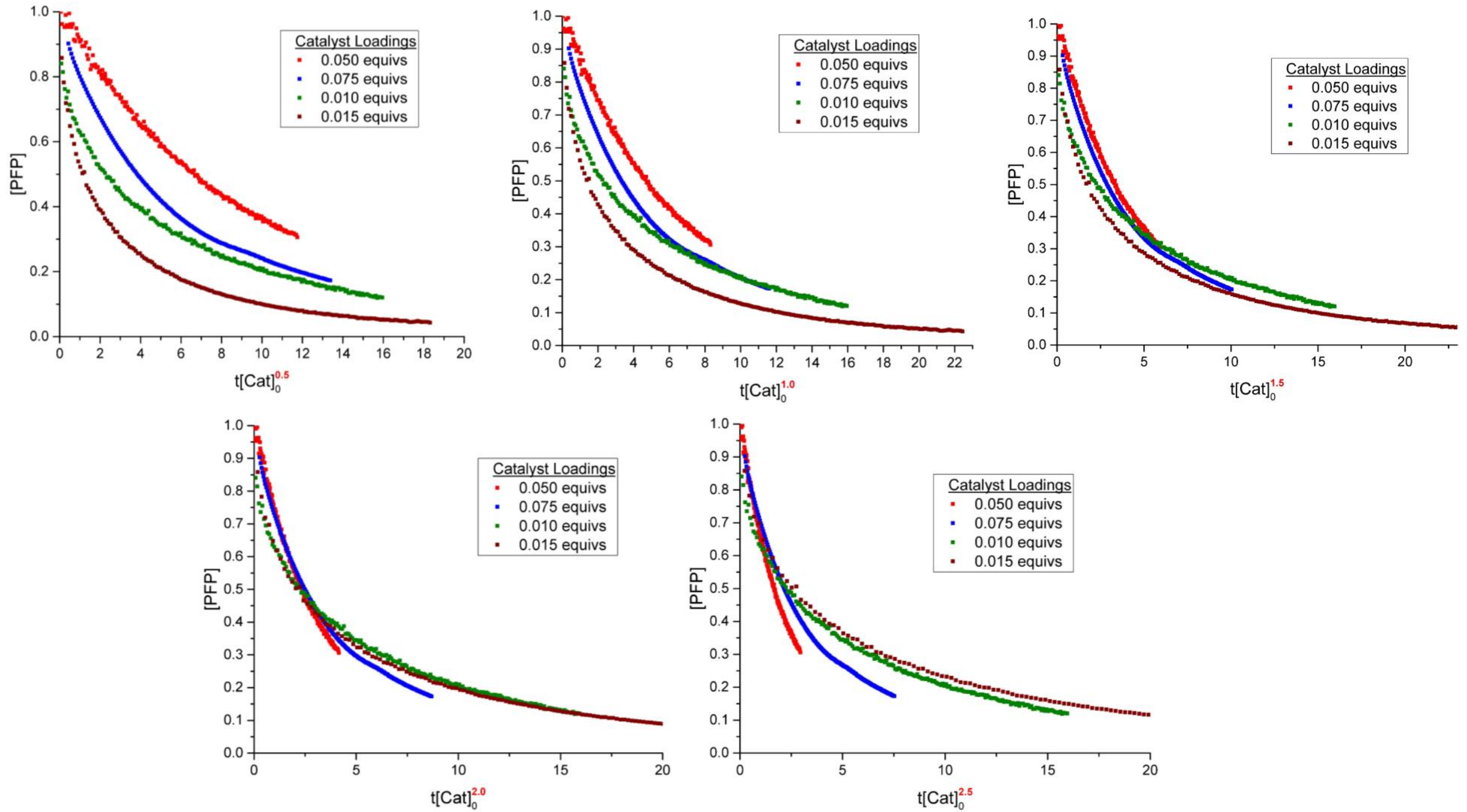

Figure S10. Graphical analysis of the order of catalyst, by plotting [PFP] against $t[\text { Cat }]^{n}$ for each catalyst loading. 


\subsection{Reaction Rate with Initiators}

Finally, to demonstrate experimentally that DMAP plays a catalytic role in the reaction, point kinetics were taken in triplicate for the reaction of pentafluoropyridine and benzoic anhydride, in the presence of 10 mol\% of DMAP, tetrabutylammonium fluoride (TBAF), or tetramethylammonium acetate (TMOAC). It is assumed that both TBAF and TMOAc simply act as initiators for the reaction.<smiles>Fc1nc(F)c(F)c(F)c1F</smiles>

[0.14 mmol]

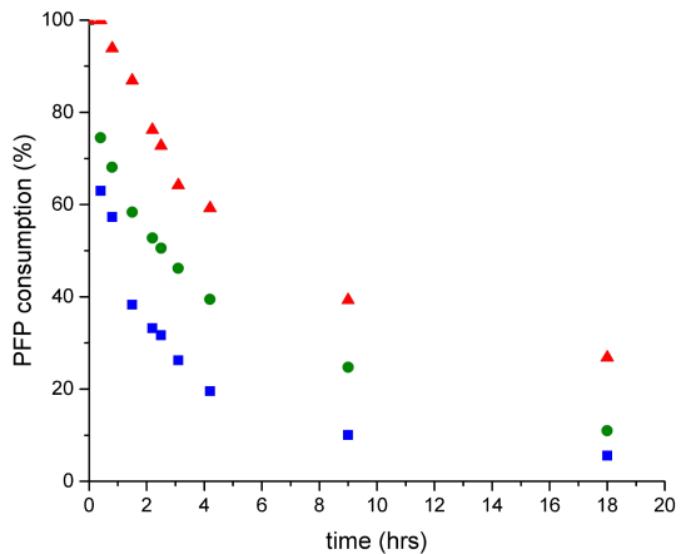

DMAP, TBAF or TMOAC ( 0.1 equivs)

$\mathrm{CH}_{3} \mathrm{CN}, 100^{\circ} \mathrm{C}$<smiles>O=C(Oc1c(F)c(F)nc(F)c1F)c1ccccc1</smiles>

2

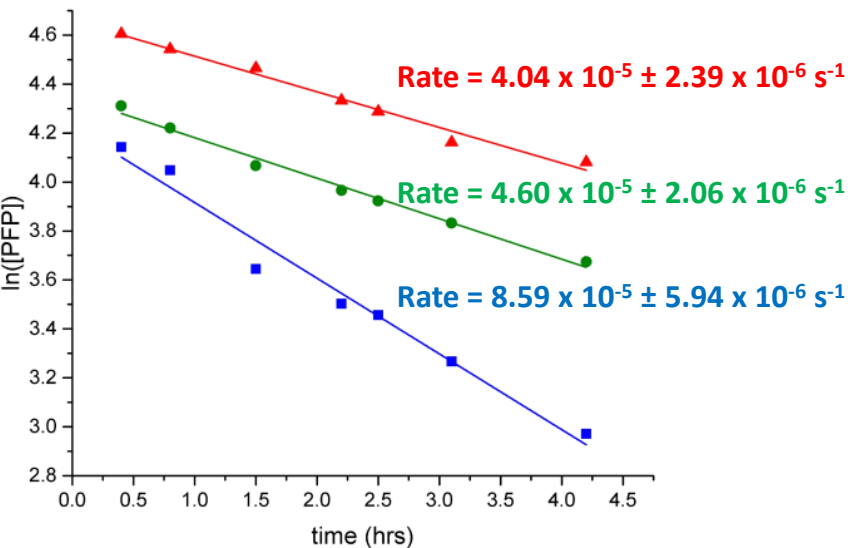

Figure S11. Comparisons for the rates of pentafluoropyridine consumption, and production of 4-benzoic-2,3,5,6tetrafluoropyridine and benzoyl fluoride, in the presence of 0.1 equivalents of DMAP, TBAF, or TMOAC.

The reaction was shown to proceed in the presence of TBAF or TMOAc, at roughly half the rate of the DMAP catalysed reaction $\left(4.60 \times 10^{-5}\right.$ and $4.04 \times 10^{-5} \mathrm{~s}^{-1}$ respectively, compared to $8.59 \times 10^{-5} \mathrm{~s}^{-1}$ for the catalysed reaction). The reaction in the presence of TMOAc showed a significant initiation period and this may be due to transesterification of acetate anions with benzoic anhydride, given no acetyl fluoride production was observed in this instance. 


\section{References}

[S1] Murray, C. B.; Sandford, G.; Korn, S. R.; Yufit, D. S.; Howard, J. A. K. J. Fluor. Chem. 2005, 126, 569.

[S2] Fox, M. A.; Sandford, G.; Slater, R.; Yufit, D. S.; Howard, J. A. K.; Vong, A. J. Fluor. Chem. 2012, $143,148$.

[S3] Senaweera, S.; Weaver, J. D. Chem. Commun. 2017, 53, 7545.

[S4] Chen, Y.; Qi, H.; Chen, N.; Ren, D.; Xu, J.; Yang, Z. J. Org. Chem. 2019, 84, 9044.

[S5] Scattolin, T.; Klein, A.; Schoenebeck, F. Org. Lett. 2017, 19, 1831.

[S6] Timperley, C. M.; Banks, R. E.; Young, I. M.; Haszeldine, R. N. J. Fluor. Chem. 2011, 132, 541.

[S7] Arisawa, M.; Suzuki, T.; Ishikawa, T.; Yamaguchi, M. J. Am. Chem. Soc. 2008, 130, 12214.

[S8] Krishnan, R.; Parthiban, A. J. Fluor. Chem. 2014, 162, 17.

[S9] Forth, P. J.; Malig, T. C.; Yu, H.; Bolduc, T. G.; Hein J. E.; Sammis, G. M. Org. Lett. 2020, 22, 6682-6686

[S10] Morgan, P. J.; Hanson-Heine, M. W. D.; Thomas, H. P.; Saunders, G. C.; Marr, A. C.; Licence, P. Organometallics 2020, 39, 2116.

[S11] Arisawa, M.; Yamada, T.; Yamaguchi, M. Tetrahedron Lett. 2010, 51, 6090.

[S12] Cismesia, M. A.; Ryan, S. J.; Bland, D. C.; Sanford, M. S. J. Org. Chem. 2017, 82, 5020.

[S13] SHELXTL v5.1, Bruker AXS, Madison, WI, 1998.

[S14] SHELX-2013, G.M. Sheldrick, Acta Cryst., 2015, C71, 3-8. 


\section{$7 \mathrm{XYZ}$ Coordinates}

TS1.log

SCF $(w B 97 x)=-1126.73767301$

$E(S C F)+Z P E(0 K)=\quad-1126.526039$

$H(298 K)=-1126.507080$

$\mathrm{G}(298 \mathrm{~K})=\quad-1126.574646$

Lowest Frequency $=-235.9677 \mathrm{~cm}-1$

$\begin{array}{llrc}\text { C } & 1.330880 & -0.375572 & -1.366967 \\ \text { C } & 2.140019 & 0.452368 & -2.109994 \\ \text { C } & 2.691513 & 1.696719 & -0.337287 \\ \text { C } & 1.918097 & 0.947879 & 0.518834 \\ \text { C } & 1.114413 & -0.118247 & 0.018534 \\ \text { N } & 2.832024 & 1.479050 & -1.637177 \\ \text { F } & 0.992003 & -1.224378 & 0.833135 \\ \text { F } & 0.646353 & -1.396573 & -1.935631 \\ \text { F } & 2.269617 & 0.196603 & -3.426036 \\ \text { F } & 3.390439 & 2.726660 & 0.177466 \\ \text { F } & 1.819548 & 1.250355 & 1.835338 \\ \text { C } & -2.868651 & 0.145466 & 1.102640 \\ \text { C } & -1.563127 & -0.279838 & 0.988124 \\ \text { C } & -1.038465 & 1.469381 & -0.439840 \\ \text { C } & -2.318987 & 1.969047 & -0.387820 \\ \text { C } & -3.300069 & 1.307863 & 0.404367 \\ \text { H } & -3.545352 & -0.420344 & 1.727342 \\ \text { H } & -1.206370 & -1.160354 & 1.506690 \\ \text { H } & -0.267129 & 1.950124 & -1.034010 \\ \text { H } & -2.555639 & 2.859531 & -0.952549 \\ \text { N } & -0.661528 & 0.369073 & 0.230224 \\ \text { N } & -4.572610 & 1.760632 & 0.488165 \\ \text { C } & -5.549566 & 1.055451 & 1.312921 \\ \text { H } & -6.503714 & 1.575601 & 1.254337 \\ \text { H } & -5.696031 & 0.027006 & 0.964702 \\ \text { H } & -5.237085 & 1.027792 & 2.362576 \\ \text { C } & -4.977774 & 2.954150 & -0.249147 \\ \text { H } & -6.030015 & 3.150915 & -0.053095 \\ \text { H } & -4.399801 & 3.830594 & 0.064112 \\ \text { H } & -4.848945 & 2.817872 & -1.328627 \\ & & & \end{array}$

\section{Int1.log}

SCF $(w B 97 x)=-1126.74728233$

$E(S C F)+Z P E(0 K)=\quad-1126.533570$

$H(298 K)=-1126.514868$

$\mathrm{G}(298 \mathrm{~K})=\quad-1126.579863$

Lowest Frequency $=32.0311 \mathrm{~cm}-1$

C $\quad 0.99446500 \quad 2.41700900 \quad-0.09981500$

C $\quad 3.01003500 \quad 3.20896500 \quad-0.68811500$

C $\quad 2.66062700 \quad 4.51985600 \quad-0.39050900$ $\begin{array}{llll}\text { C } & 0.51723700 & 3.68216000 & 0.20944200\end{array}$

N $\quad 2.21044000 \quad 2.17327400 \quad-0.54310900$

C $\quad 1.39553800 \quad 7.24104000 \quad-0.33702200$

$\begin{array}{lllll}\text { C } & 0.04858400 & 6.29487600 & 1.45874500\end{array}$

C $\quad 0.35340000 \quad 8.28273300 \quad-0.45796200$

H $\quad 1.76722300 \quad 6.91673600 \quad-1.30772600$

C $\quad-0.65614300 \quad 7.43672200 \quad 1.56873900$

H $\quad-0.00301000 \quad 5.50269800 \quad 2.19285600$

C $\quad-0.59341000 \quad 8.44019300 \quad 0.51415000$

H $\quad 0.44067400 \quad 8.95308500 \quad-1.30104600$

H $\quad-1.26062000 \quad 7.59079400 \quad 2.45168600$

C $\quad 1.36430800 \quad 4.79983300 \quad 0.07968000$

F $\quad 4.24822100 \quad 2.97063400 \quad-1.13221800$

F $\quad \begin{array}{llll}0.16844300 & 1.37466000 & 0.03427300\end{array}$

F $\quad-0.75787200 \quad 3.82589600 \quad 0.60421300$

F $\quad 3.57217200 \quad 5.48892600 \quad-0.55383300$

N $\quad 0.91915200 \quad 6.08285000 \quad 0.38782400$

N $\quad \begin{array}{llll}-1.47666200 & 9.51352900 & 0.56565800\end{array}$

C $\quad-1.34012000 \quad 10.53934100 \quad-0.45635000$

H $\quad-1.97692200 \quad 11.38665700 \quad-0.19271200$

H $\quad-0.30432700 \quad 10.88416900-0.50272400$

H $\quad-1.63401900 \quad 10.18537700 \quad-1.45752200$

$\begin{array}{lllll}\text { C } & -2.86571300 & 9.27170100 & 0.95327100\end{array}$

H $\quad-3.31701500 \quad 10.21397100 \quad 1.27450600$

H $\quad-3.46006100 \quad 8.87015000 \quad 0.11810300$

H $\quad-2.92708200 \quad 8.56754100 \quad 1.78132000$

F $\quad \begin{array}{llll}2.53856900 & 7.75931600 & 0.35280100\end{array}$

\section{TS2.log}

SCF $(w B 97 x)=-764.058041912$

$E(S C F)+Z P E(0 K)=\quad-763.794737$

$\mathrm{H}(298 \mathrm{~K})=\quad-763.777195$

$\mathrm{G}(298 \mathrm{~K})=\quad-763.839816$

Lowest Frequency $=-111.0737 \mathrm{~cm}-1$

C $\quad-4.390718 \quad-1.420517 \quad 0.600023$

$\begin{array}{llll}\text { O } & -3.767722 & -2.260171 & 1.265112\end{array}$

O $\quad-4.035236-1.232191-0.847727$

C $\quad-3.731249-2.238820-1.695035$

$\begin{array}{lllll}\text { O } & -3.230647 & -1.949519 & -2.768301\end{array}$

C $\quad-7.739120-3.483375 \quad 1.174393$

C $\quad-6.466352-2.959157 \quad 1.211819$

$\begin{array}{llll}\text { C } & -6.936441 & -1.469739 & -0.503877\end{array}$

C $\quad-8.227787-1.932355-0.616703$

$\begin{array}{llll}\text { C } & -8.683927 & -2.974459 & 0.240249\end{array}$

H $\quad-7.995579-4.280081 \quad 1.858228$

H $\quad-5.696984-3.316365 \quad 1.888588$

H $\quad-6.555085-0.688408-1.151016$

H $\quad-8.873582-1.494118-1.364313$

N $\quad-6.070418 \quad-1.972632 \quad 0.394705$

N $\quad-9.947141 \quad-3.456359 \quad 0.165669$

H $\quad-11.412954 \quad-4.7782250 .839336$ 


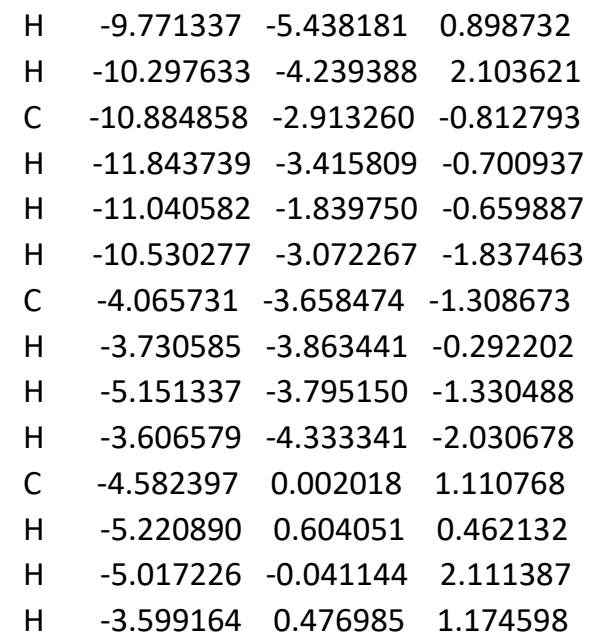

\section{Int2.log}

$\mathrm{SCF}(\mathrm{wB97x})=-764.062234832$

$E(S C F)+Z P E(0 ~ K)=\quad-763.799199$

$H(298 K)=\quad-763.779929$

$\mathrm{G}(298 \mathrm{~K})=\quad-763.849214$

Lowest Frequency $=13.3194 \mathrm{~cm}-1$

$\begin{array}{lrrr}\text { C } & 0.481390 & 1.859104 & 0.216609 \\ \text { C } & 1.259542 & 2.953782 & -0.015124 \\ \text { C } & 0.757531 & 4.269281 & 0.210601 \\ \text { C } & -0.593116 & 4.364685 & 0.681908 \\ \text { C } & -1.328965 & 3.239975 & 0.890493 \\ \text { H } & 0.891168 & 0.858854 & 0.011293 \\ \text { H } & 2.252931 & 2.733195 & -0.390125 \\ \text { H } & -1.056235 & 5.320734 & 0.879157 \\ \text { H } & -2.351610 & 3.256762 & 1.239505 \\ \text { N } & -0.809958 & 1.988360 & 0.667432 \\ \text { N } & 1.506072 & 5.357273 & -0.009934 \\ \text { C } & 2.881396 & 5.214406 & -0.496932 \\ \text { H } & 2.899681 & 4.708935 & -1.466720 \\ \text { H } & 3.319171 & 6.203483 & -0.609995 \\ \text { H } & 3.486324 & 4.641541 & 0.211648 \\ \text { C } & 0.965259 & 6.698422 & 0.225601 \\ \text { H } & 0.677246 & 6.824661 & 1.273468 \\ \text { H } & 1.730770 & 7.433196 & -0.012548 \\ \text { H } & 0.094545 & 6.885859 & -0.409866 \\ \text { C } & -1.665067 & 0.837953 & 0.906882 \\ \text { O } & -2.814921 & 1.031464 & 1.231370 \\ \text { C } & 2.936411 & -0.187518 & -1.125660 \\ \text { O } & 3.374677 & 0.992109 & -1.090004 \\ \text { O } & 1.837814 & -0.610003 & -0.647685 \\ \text { C } & -1.034176 & -0.513632 & 0.753724 \\ \text { H } & -0.539226 & -0.640833 & -0.211637 \\ \text { H } & -0.259580 & -0.650767 & 1.514828 \\ \text { H } & -1.814727 & -1.260596 & 0.888669 \\ \mathrm{C} & 3.813573 & -1.254243 & -1.810458\end{array}$

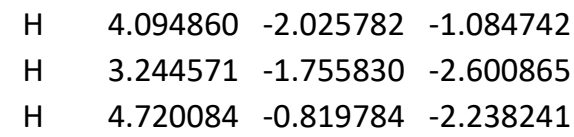

TS3.log

SCF $(w B 97 x)=-635.375146067$

$E(S C F)+Z P E(0 K)=\quad-635.162622$

$H(298 K)=-635.148269$

$\mathrm{G}(298 \mathrm{~K})=\quad-635.203191$

Lowest Frequency $=-145.7197 \mathrm{~cm}-1$

$\begin{array}{llll}\text { C } & 0.647137 & 1.808953 & 0.686427\end{array}$

$\begin{array}{llll}\text { C } & 1.372362 & 2.928061 & 0.373685\end{array}$

C $\quad 0.759484 \quad 4.214865 \quad 0.320045$

$\begin{array}{llll}\text { C } & -0.628092 & 4.273223 & 0.654989\end{array}$

$\begin{array}{llll}\text { C } & -1.305920 & 3.127796 & 0.963451\end{array}$

$\begin{array}{llll}\text { H } & 2.432574 & 2.812353 & 0.197960\end{array}$

H $\quad-1.168366 \quad 5.208486 \quad 0.669543$

H $\quad-2.356634 \quad 3.122980 \quad 1.221597$

$\begin{array}{lllll}\mathrm{N} & -0.689071 & 1.914824 & 0.949204\end{array}$

$\begin{array}{llll}\text { N } & 1.451587 & 5.320527 & -0.011627\end{array}$

$\begin{array}{llll}\text { C } & 2.874360 & 5.228802 & -0.340088\end{array}$

H $\quad 3.038586 \quad 4.540995 \quad-1.175077$

H $\quad 3.233270 \quad 6.213662 \quad-0.631106$

$\begin{array}{llll}H & 3.459008 & 4.886499 & 0.520351\end{array}$

$\begin{array}{lllll}\text { C } & 0.799604 & 6.630398 & -0.013844\end{array}$

$\begin{array}{llll}H & 0.433984 & 6.892139 & 0.984473\end{array}$

H $\quad 1.521294 \quad 7.383051 \quad-0.323872$

H $\quad-0.040424 \quad 6.649320 \quad-0.715183$

$\begin{array}{llll}\text { C } & -1.517992 & 0.709526 & 1.224278\end{array}$

$\begin{array}{lllll}\text { O } & -2.312805 & 0.786665 & 2.129287\end{array}$

$\begin{array}{llll}\text { C } & -1.422937 & -0.371236 & 0.192154\end{array}$

H $\quad-0.470835 \quad-0.867507 \quad 0.415523$

H $\quad-2.262749-1.054870 \quad 0.322056$

H $\quad-1.424860 \quad 0.051962 \quad-0.815899$

$\begin{array}{llll}\mathrm{H} & 1.014891 & 0.785597 & 0.923024\end{array}$

F $\quad 0.632632 \quad-0.451807 \quad 1.914774$

Int3.log

SCF $(w B 97 x)=-1255.41990278$

$E(S C F)+Z P E(0 K)=\quad-1255.157410$

$H(298 K)=\quad-1255.133784$

$G(298 K)=\quad-1255.211452$

Lowest Frequency $=26.9732 \mathrm{~cm}-1$

$\begin{array}{llll}\text { C } & 1.382468 & 2.738610 & -1.409951\end{array}$

$\begin{array}{llll}\text { C } & 3.194791 & 3.682672 & -0.440745\end{array}$

C $\quad 2.499413 \quad 4.840695 \quad-0.118973$

C $\quad 0.577647 \quad 3.838748-1.130478$

N $\quad 2.651129 \quad 2.663680 \quad-1.073888$

$\begin{array}{llll}\text { C } & -0.203038 & 6.751256 & -1.250648\end{array}$ 


$\begin{array}{cccc}\text { C } & 0.305506 & 6.582804 & 1.068535 \\ \text { C } & -0.923568 & 7.889316 & -1.022593 \\ \text { H } & -0.021230 & 6.312117 & -2.238862 \\ \text { C } & -0.395148 & 7.718685 & 1.341673 \\ \text { H } & 0.807672 & 6.012422 & 1.837741 \\ \text { C } & -1.056120 & 8.429842 & 0.291224 \\ \text { H } & -1.381228 & 8.371882 & -1.874036 \\ \text { H } & -0.437648 & 8.055014 & 2.366868 \\ \text { C } & 1.153281 & 4.920727 & -0.470258 \\ \text { F } & 4.488158 & 3.595199 & -0.119611 \\ \text { F } & 0.846927 & 1.698770 & -2.053071 \\ \text { F } & -0.708511 & 3.854365 & -1.480592 \\ \text { F } & 3.103766 & 5.860509 & 0.493598 \\ \text { N } & 0.398166 & 6.096031 & -0.206654 \\ \text { N } & -1.760868 & 9.545301 & 0.533577 \\ \text { C } & -2.415162 & 10.258836 & -0.565572 \\ \text { H } & -2.920643 & 11.134508 & -0.165043 \\ \text { H } & -1.682095 & 10.590310 & -1.307188 \\ \text { H } & -3.157797 & 9.623381 & -1.057587 \\ \text { C } & -1.880684 & 10.067689 & 1.896956 \\ \text { H } & -2.506206 & 10.957071 & 1.878464 \\ \text { H } & -2.347174 & 9.331145 & 2.558123 \\ \text { H } & -0.900780 & 10.340872 & 2.300529 \\ \text { C } & 2.061418 & 5.644888 & -3.427862 \\ \text { O } & 2.464540 & 6.481631 & -2.576763 \\ \text { O } & 0.852751 & 5.325566 & -3.650891 \\ \text { C } & 3.130062 & 4.878323 & -4.229278 \\ \text { H } & 3.404406 & 3.971988 & -3.675296 \\ \text { H } & 2.749904 & 4.565341 & -5.205357 \\ \text { H } & 4.036961 & 5.475785 & -4.354947\end{array}$

$\begin{array}{cccc}\text { H } & -0.402785 & 8.120354 & 2.214026 \\ \text { C } & 1.549936 & 5.324460 & -0.819812 \\ \text { F } & 3.874923 & 3.435016 & 1.270412 \\ \text { F } & 0.437485 & 1.864980 & -1.251895 \\ \text { F } & -0.286275 & 4.365579 & -2.012488 \\ \text { F } & 3.317348 & 6.006423 & 0.641995 \\ \text { N } & 0.720562 & 6.526845 & -0.501202 \\ \text { N } & -1.843435 & 9.641586 & 0.491403 \\ \text { C } & -2.568472 & 10.362911 & -0.555708 \\ \text { H } & -3.164608 & 11.147189 & -0.094690 \\ \text { H } & -1.874868 & 10.826727 & -1.264023 \\ \text { H } & -3.239865 & 9.692988 & -1.102260 \\ \text { C } & -2.031129 & 10.026464 & 1.891160 \\ \text { H } & -2.721416 & 10.865847 & 1.935941 \\ \text { H } & -2.451898 & 9.200441 & 2.473254 \\ \text { H } & -1.083170 & 10.333468 & 2.343761 \\ \text { C } & 3.136548 & 5.328594 & -2.886646 \\ \text { O } & 3.433514 & 4.163900 & -2.652173 \\ \text { O } & 2.289301 & 6.069041 & -2.189798 \\ \text { C } & 3.723471 & 6.108357 & -4.052171 \\ \text { H } & 2.925270 & 6.412297 & -4.736223 \\ \text { H } & 4.207054 & 7.019875 & -3.689379 \\ \text { H } & 4.447847 & 5.491450 & -4.585309\end{array}$

\section{TS4.log}

SCF $(w B 97 x)=-1255.39350023$

$E(S C F)+Z P E(0 K)=-1255.131857$

$\mathrm{H}(298 \mathrm{~K})=-1255.109076$

$\mathrm{G}(298 \mathrm{~K})=-1255.184298$

Lowest Frequency $=-225.3298 \mathrm{~cm}-1$
$\begin{array}{llll}\text { C } & 1.161848 & 2.912497 & -0.806459\end{array}$
C $\quad 2.842004 \quad 3.6824810 .438900$
$\begin{array}{llll}\text { C } & 2.554470 & 4.991152 & 0.146256\end{array}$
$\begin{array}{lllll}C & 0.778373 & 4.170527 & -1.186540\end{array}$
$\begin{array}{llll}\mathrm{N} & 2.177796 & 2.625743 & -0.003390\end{array}$
$\begin{array}{llll}\text { C } & 0.037880 & 7.192646 & -1.474164\end{array}$
$\begin{array}{lllll}\text { C } & 0.524946 & 6.871164 & 0.800879\end{array}$
$\begin{array}{lllll}\text { C } & -0.816249 & 8.218794 & -1.178911\end{array}$
H $\quad 0.223290 \quad 6.866670 \quad-2.484033$
$\begin{array}{llll}\text { C } & -0.303640 & 7.895560 & 1.162228\end{array}$
H $\quad 1.062923 \quad 6.287650 \quad 1.533541$
$\begin{array}{llll}\text { C } & -1.020528 & 8.629039 & 0.170906\end{array}$
H $\quad-1.326158 \quad 8.703248 \quad-1.998642$ 
1a. 4-benzoic-2,3,5,6-tetrafluoropyridine $1 \mathrm{H}$ C6D6, $400 \mathrm{MHz}$, 298K.<smiles>O=C(Oc1c(F)c(F)nc(F)c1F)c1ccccc1</smiles>
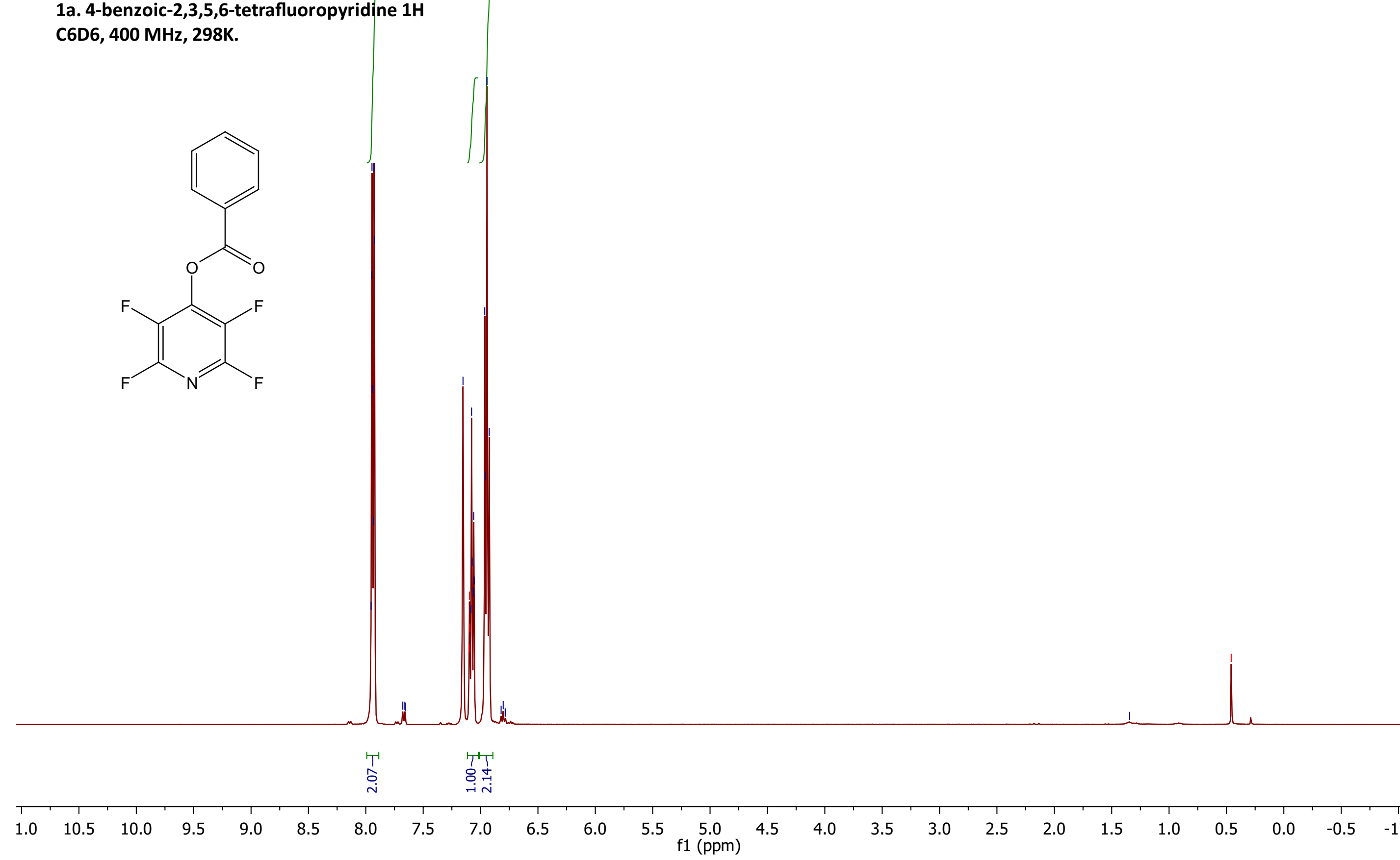


\section{1a. 4-benzoic-2,3,5,6-tetrafluoropyridine $13 \mathrm{C}$} C6D6, $100 \mathrm{MHz}, 298 \mathrm{~K}$.<smiles>O=C(Oc1c(F)c(F)nc(F)c1F)c1ccccc1</smiles>

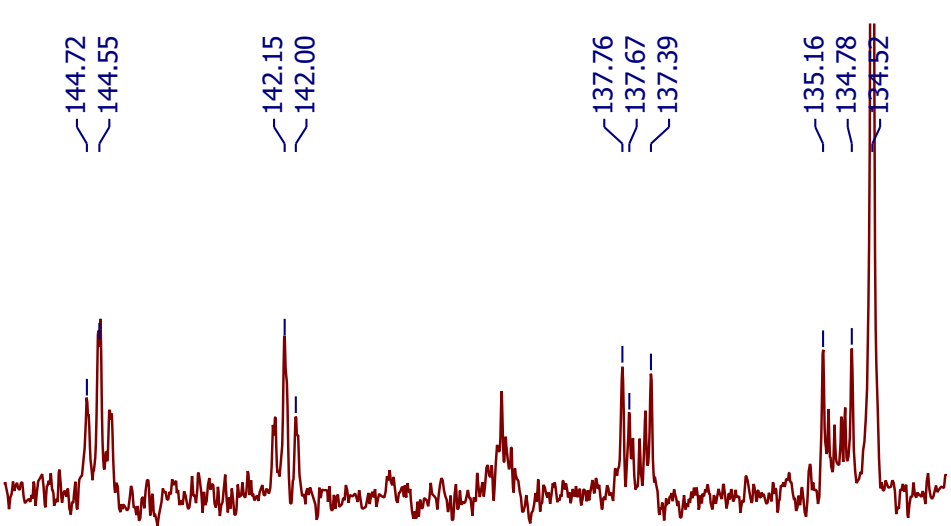

$\begin{array}{llllllllllll}145 & 144 & 143 & 142 & 141 & 140 & 139 & 138 & 137 & 136 & 135 & 134\end{array}$ $\mathrm{f} 1$ (ppm) 
C6D6, $100 \mathrm{MHz}$, C6D6

\section{1a. 4-benzoic-2,3,5,6-tetrafluoropyridine 19F}

\section{CGD6, $100 \mathrm{MHz}, \mathrm{CGDG}$}<smiles>O=C(Oc1c(F)c(F)nc(F)c1F)c1ccccc1</smiles>
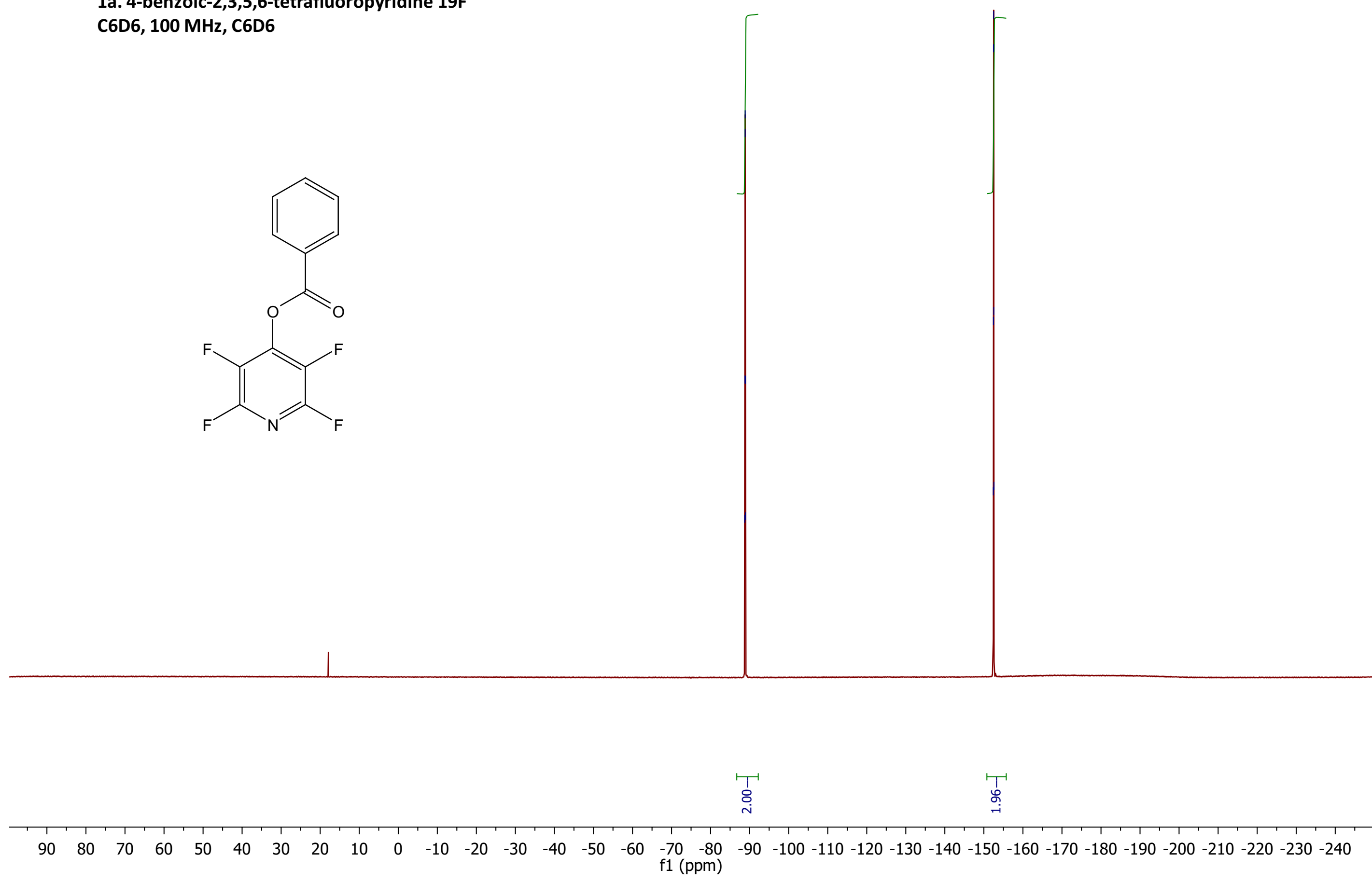
1b. 4-acetyl-2,3,5,6-tetrafluoropyridine, $1 \mathrm{H}$ C6D6 400 MHz, $298 \mathrm{~K}$.<smiles>CC(=O)Oc1c(F)c(F)nc(F)c1F</smiles>

\begin{tabular}{|c|c|c|c|c|c|c|c|c|c|c|c|c|c|c|c|c|c|c|}
\hline & & & & & & & & & & & & & & & †! & & & \\
\hline 9.5 & 9.0 & 8.5 & 8.0 & 7.5 & 7.0 & 6.5 & 6.0 & 5.5 & $\begin{array}{l}5.0 \\
\text { f1 (ppm) }\end{array}$ & 4.0 & 3.5 & 3.0 & 2.5 & 2.0 & 1.5 & 1.0 & 0.5 & 0.0 \\
\hline
\end{tabular}


1b. 4-acetyl-2,3,5,6-tetrafluoropyridine, $13 \mathrm{C}$, C6D6 $100 \mathrm{MHz}, 298 \mathrm{~K}$.

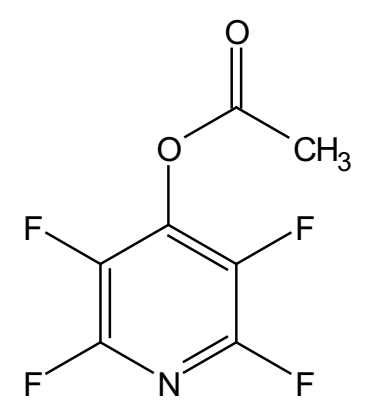

l

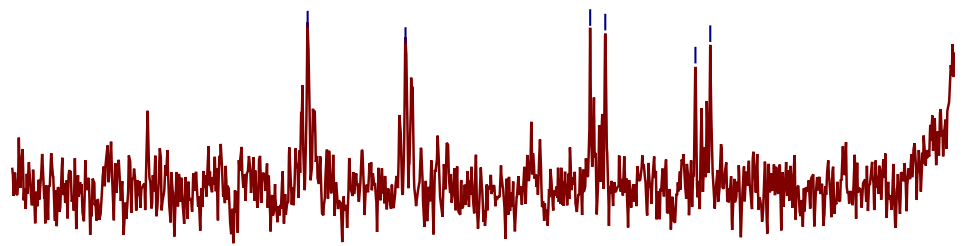

$\begin{array}{lllllllllll}150 & 148 & 146 & 144 & 142 & 140 & 138 & 136 & 134 & 132 & 130\end{array}$ f1 (ppm)

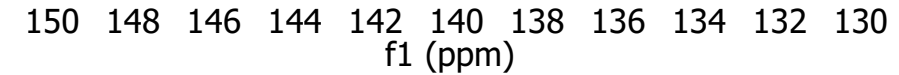

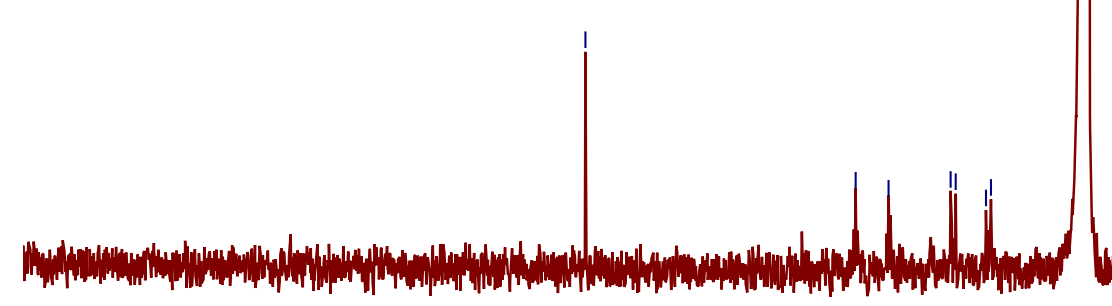

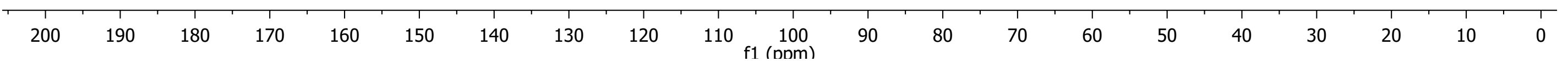


1b. 4-acetyl-2,3,5,6-tetrafluoropyridine, 19F C6D6 $100 \mathrm{MHz}, 298 \mathrm{~K}$.

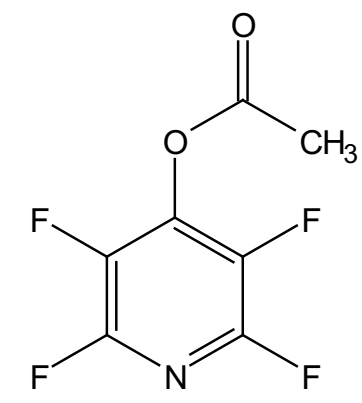

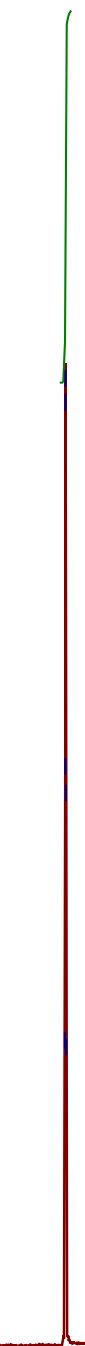

\begin{tabular}{|c|c|c|c|c|c|c|c|c|c|c|c|c|c|c|c|c|c|c|}
\hline & & & & & & & & & $\begin{array}{l}T \\
\text { ô } \\
\end{array}$ & & $\begin{array}{l}\prod^{\prime} \\
\stackrel{\leftrightarrow}{\circ}\end{array}$ & & & & & & & \\
\hline 90 & 70 & 50 & 30 & 10 & -10 & -30 & -50 & -70 & $\begin{array}{l}-90 \quad-110 \\
\mathrm{f} 1(\mathrm{ppm})\end{array}$ & -130 & -150 & -170 & -190 & -210 & -230 & -250 & -270 & -290 \\
\hline
\end{tabular}


1c. 4-acetyl-2,3,5,6-tetrafluoropyridine, $1 \mathrm{H}$,

C6D6, $400 \mathrm{MHz}, 298 \mathrm{~K}$.<smiles>C/C=C/C(=O)Oc1c(F)c(F)nc(F)c1F</smiles>

$\mathrm{N}_{\mathrm{F}}$

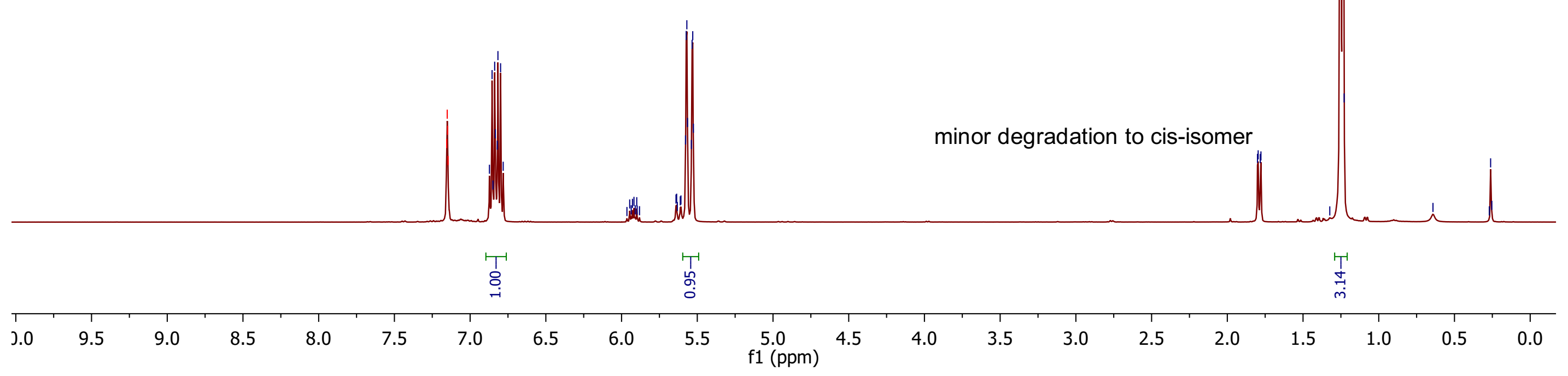


1c. 4-acetyl-2,3,5,6-tetrafluoropyridine, 13C, C6D6, $100 \mathrm{MHz}, 298 \mathrm{~K}$.

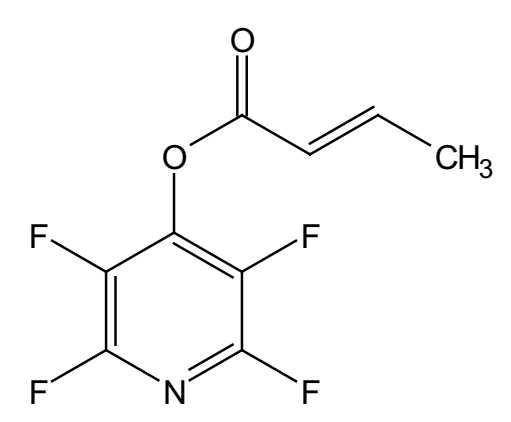

$$
\text { 亲 }
$$

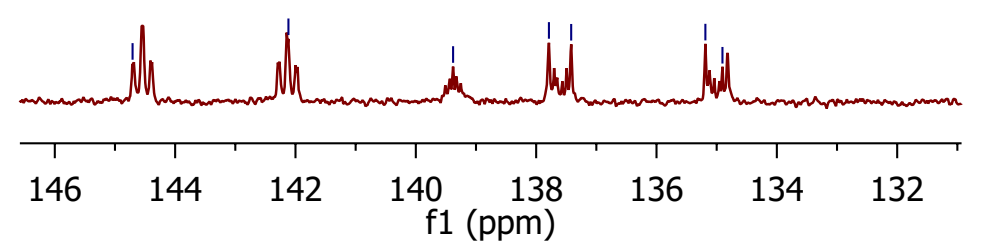

minor degradation to cis-isomer

$180 \quad 170$

160

150

140

130

120

110

100
(ppm)

90

80

70

60

50

40 
1c. 4-acetyl-2,3,5,6-tetrafluoropyridine, 19F,

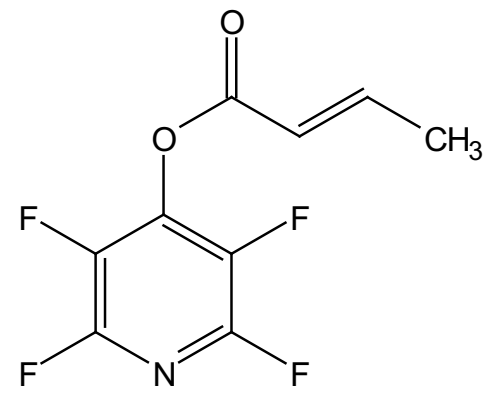


1d. 4-decanoic-2,3,5,6-tetrafluoropyridine, $1 \mathrm{H}$

C6D6, $400 \mathrm{MHz}, 298 \mathrm{~K}$
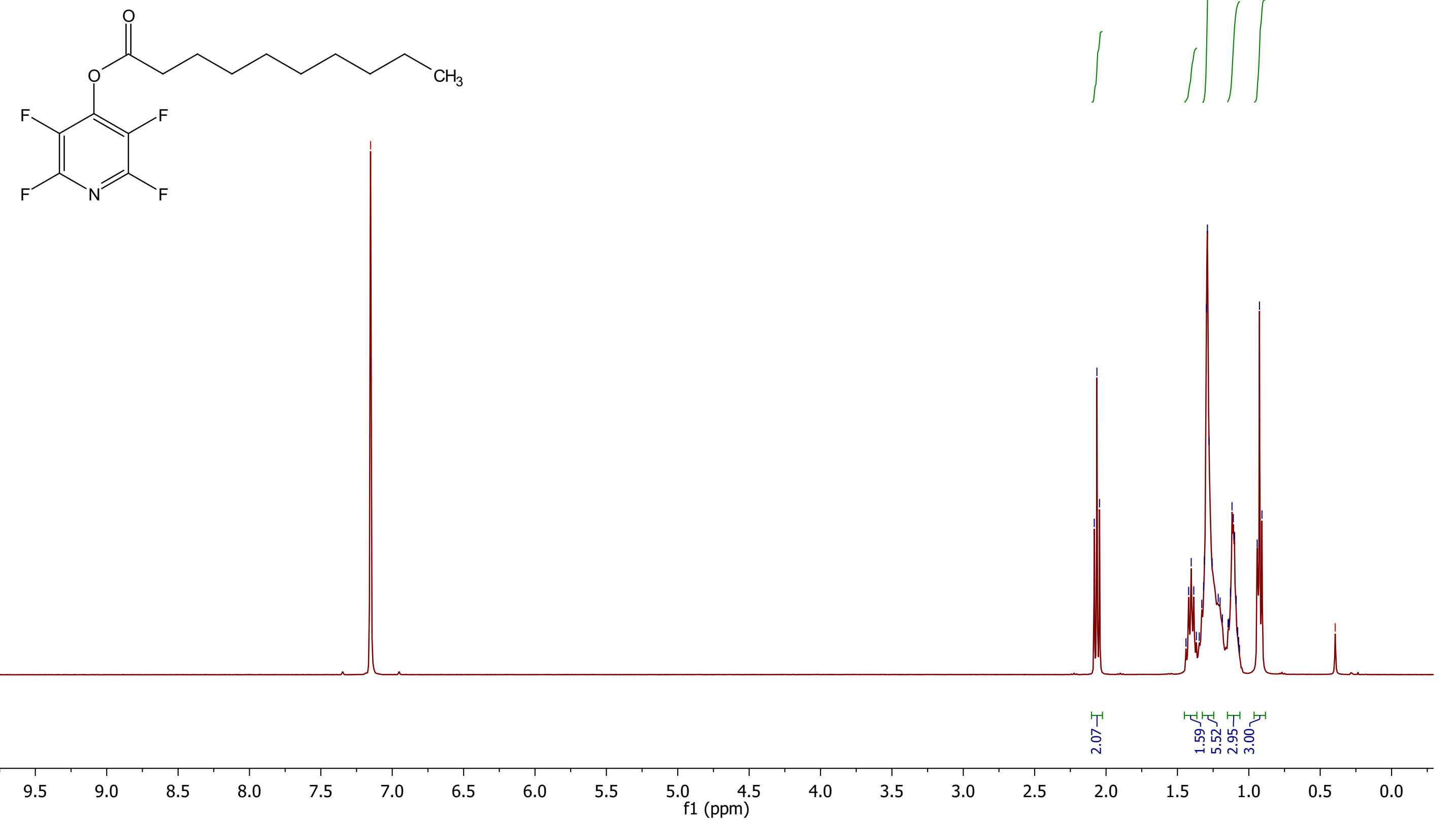
1d. 4-decanoic-2,3,5,6-tetrafluoropyridine, $13 \mathrm{C}$ C6D6, $100 \mathrm{MHz}, 298 \mathrm{~K}$

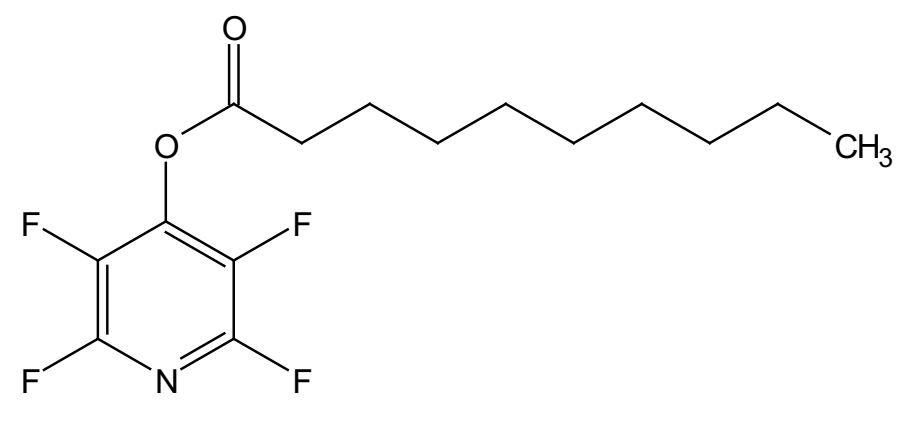

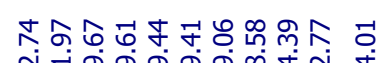

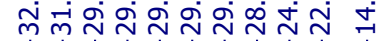

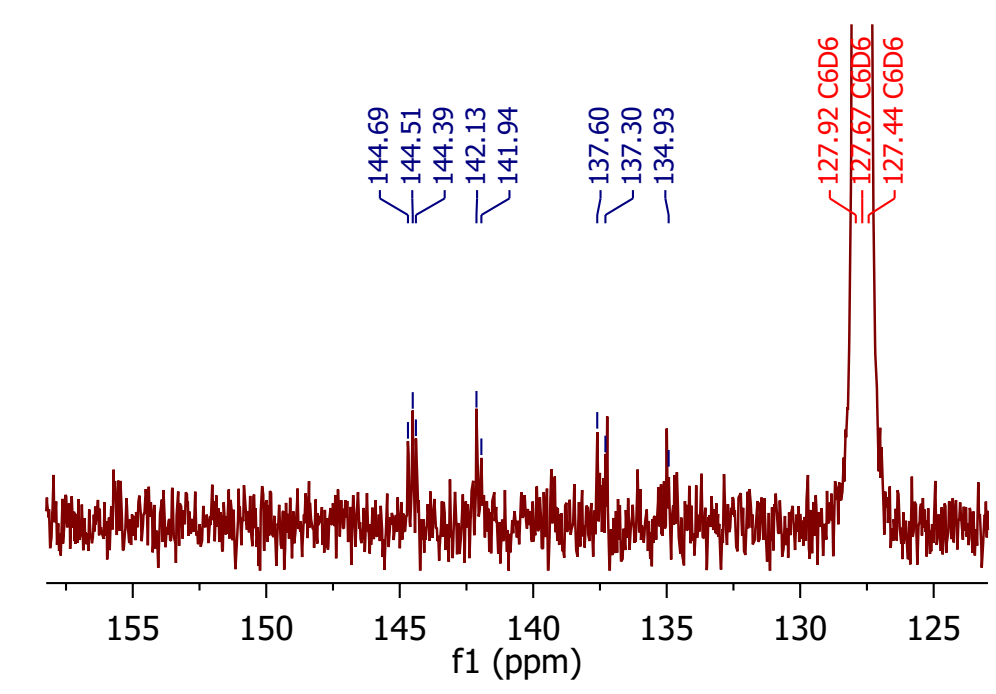


1d. 4-decanoic-2,3,5,6-tetrafluoropyridine, 19F

C6D6, $100 \mathrm{MHz}, 298 \mathrm{~K}$

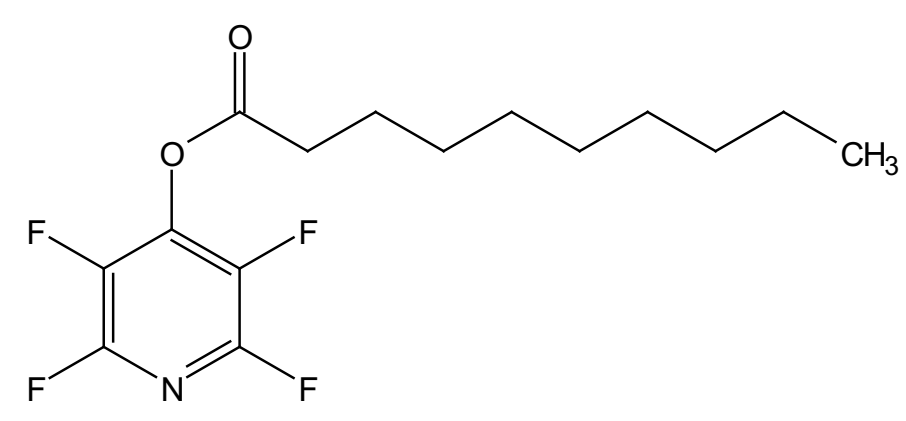

\begin{tabular}{|c|c|c|c|c|c|c|c|c|c|c|c|c|c|c|c|c|c|c|}
\hline & & & & & & & & & $\begin{array}{l}\underset{1}{T} \\
\stackrel{\leftrightarrow}{+}\end{array}$ & & $\begin{array}{l}\| \\
\stackrel{\text { O}}{\mathrm{N}}\end{array}$ & & & & & & & \\
\hline 90 & 70 & 50 & 30 & 10 & -10 & -30 & -50 & -70 & $\begin{array}{l}-90 \quad-110 \\
\mathrm{f} 1(\mathrm{ppm})\end{array}$ & -130 & -150 & -170 & -190 & -210 & -230 & -250 & -270 & -290 \\
\hline
\end{tabular}


1e. methyl-2,3,5,6-tetrafluoropyridin-4-ylcarbonate, $1 \mathrm{H}$,

C6D6, $400 \mathrm{MHz}, 298 \mathrm{~K}$.

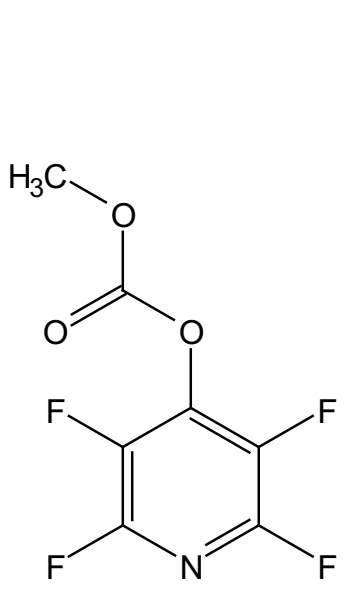

\begin{tabular}{|c|c|c|c|c|c|c|c|c|c|c|c|c|c|c|c|c|c|c|}
\hline & & & & & & & & & & & & $\begin{array}{l}1 \\
\stackrel{1}{\prime} \\
\end{array}$ & & & & & & \\
\hline 9.0 & 8.5 & 8.0 & 7.5 & 7.0 & 6.5 & 6.0 & 5.5 & 5.0 & $\begin{array}{r}4.5 \\
(\mathrm{ppm})\end{array}$ & 4.0 & 3.5 & 3.0 & 2.5 & 2.0 & 1.5 & 1.0 & 0.5 & 0.0 \\
\hline
\end{tabular}


1e. methyl-2,3,5,6-tetrafluoropyridin-4-ylcarbonate 13C, C6D6, $100 \mathrm{MHz}, 298 \mathrm{~K}$.

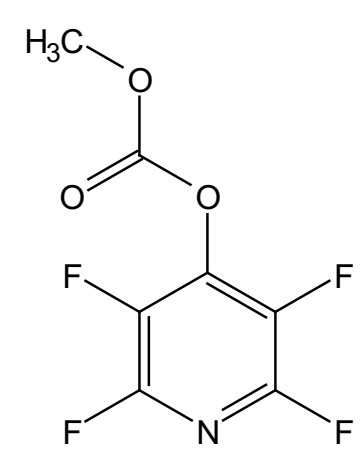

눈

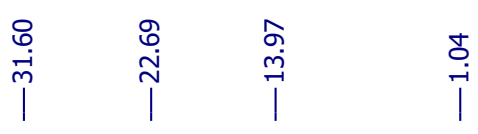

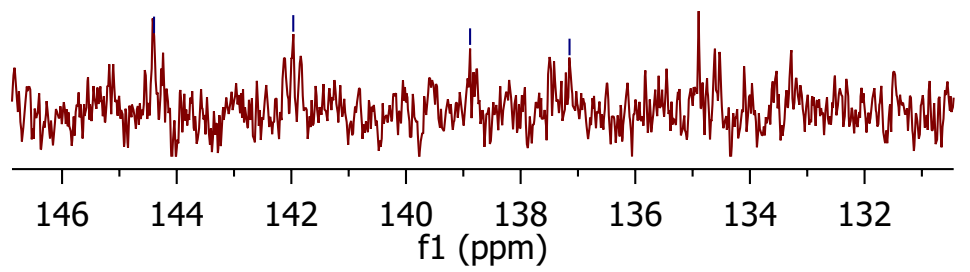

70

60


1e. methyl-2,3,5,6-tetrafluoropyridin-4-ylcarbonate, 19F, C6D6, $100 \mathrm{MHz}, 298 \mathrm{~K}$.
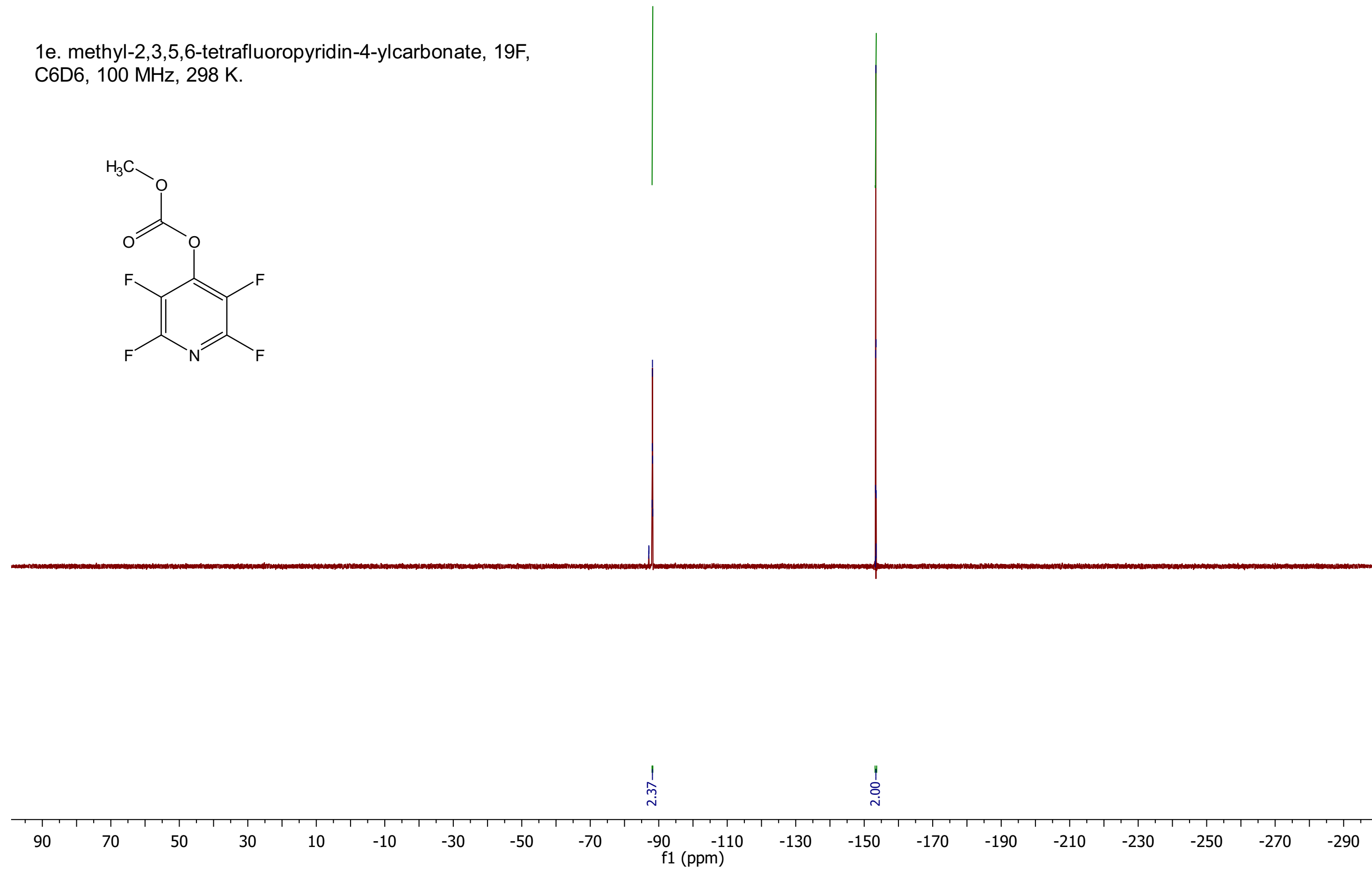


\section{1f. 4-phenylthio-2,3,5,6-tetrafluoropyridine, $1 \mathrm{H}$}

C6D6, $400 \mathrm{MHz}, 298 \mathrm{~K}$.
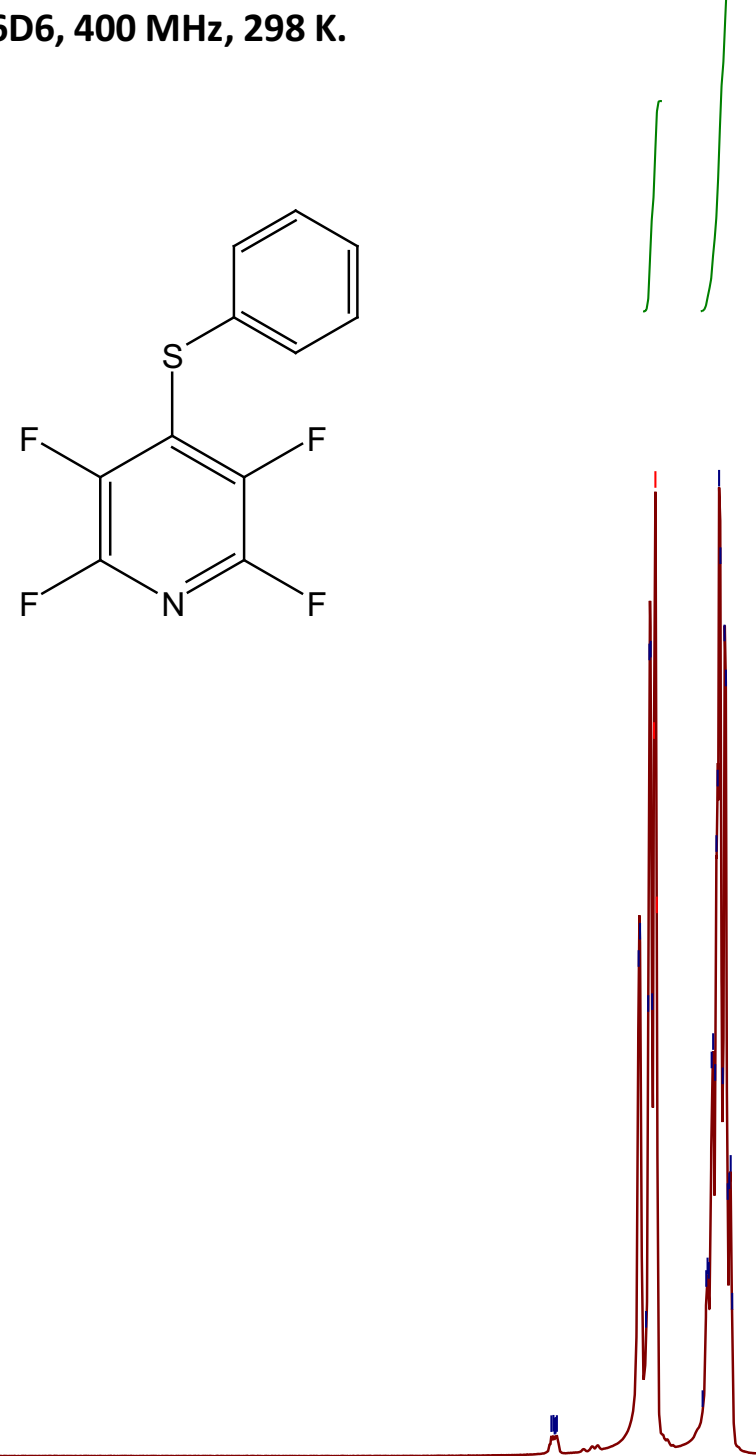

西
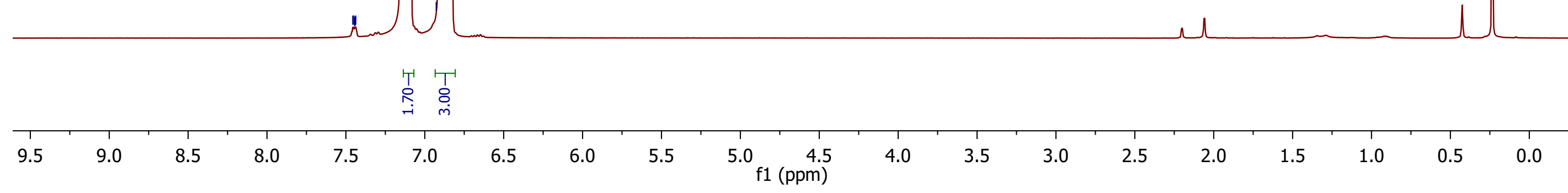
1f. 4-phenylthio-2,3,5,6-tetrafluoropyridine, 13C C6D6, $100 \mathrm{MHz}, 298 \mathrm{~K}$.

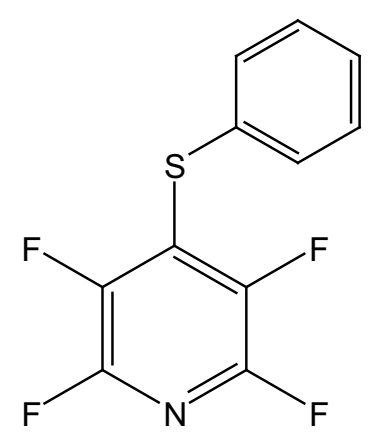

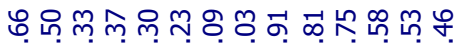

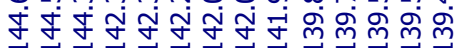
41

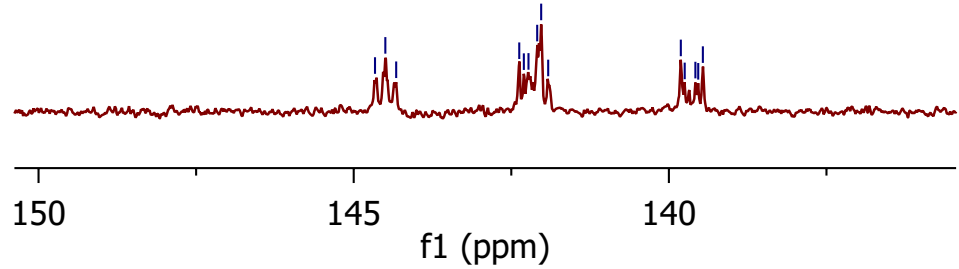

dild 


\section{1f. 4-phenylthio-2,3,5,6-tetrafluoropyridine, 19F}

C6D6, $100 \mathrm{MHz}, 298 \mathrm{~K}$.<smiles>Fc1nc(F)c(F)c(Sc2ccccc2)c1F</smiles>

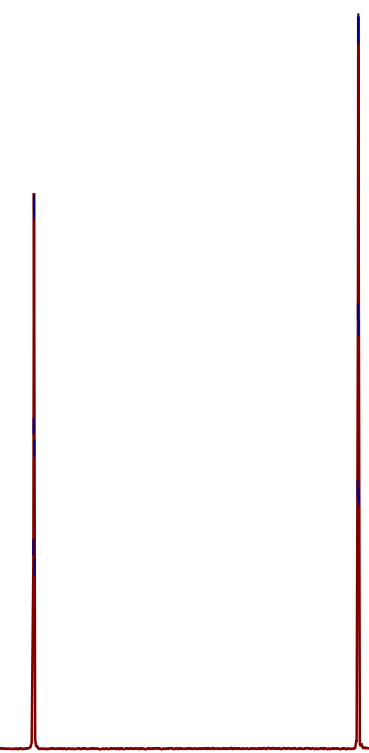

\begin{tabular}{|c|c|c|c|c|c|c|c|c|c|c|c|c|c|c|c|c|c|c|c|}
\hline & & & & & & & & & 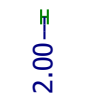 & & & & & & & & & & \\
\hline 90 & 70 & 50 & 30 & 10 & -10 & -30 & -50 & -70 & $\begin{array}{r}-90 \\
\quad f 1\end{array}$ & $\begin{array}{l}-110 \\
\mathrm{~m})\end{array}$ & -130 & -150 & -170 & -190 & -210 & -230 & -250 & -270 & -290 \\
\hline
\end{tabular}


1h. 4-thiocyanato-2,3,5,6-tetrafluorpyridine,

$13 \mathrm{C}$,

C6D6, $100 \mathrm{MHz}, 298 \mathrm{~K}$.

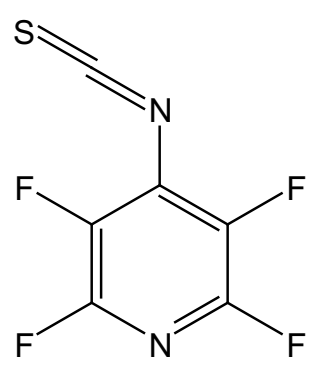


1h. 4-thiocyanato-2,3,5,6-tetrafluorpyridine, 19F, C6D6, $100 \mathrm{MHz}, 298 \mathrm{~K}$.
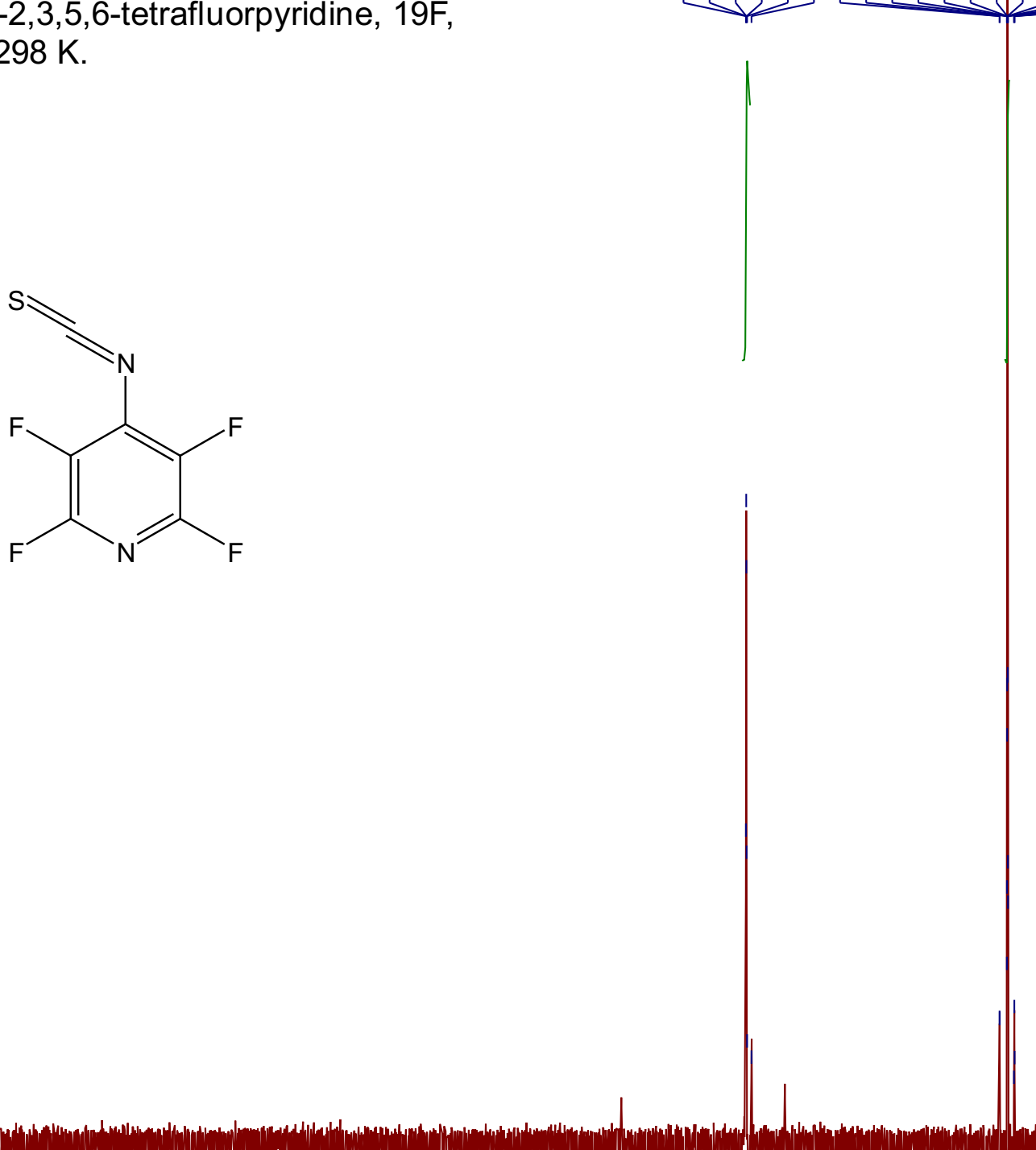

\begin{tabular}{|c|c|c|c|c|c|c|c|c|c|c|c|c|c|c|c|c|c|c|}
\hline & & & & & & & & & 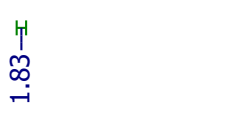 & $\begin{array}{l}t \\
\stackrel{1}{0} \\
\text { in }\end{array}$ & & & & & & & & \\
\hline 90 & 70 & 50 & 30 & 10 & -10 & -30 & -50 & -70 & $\begin{array}{ll}-90 & -110 \\
\mathrm{f} 1(\mathrm{ppm}) & \end{array}$ & -130 & -150 & -170 & -190 & -210 & -230 & -250 & -270 & -290 \\
\hline
\end{tabular}




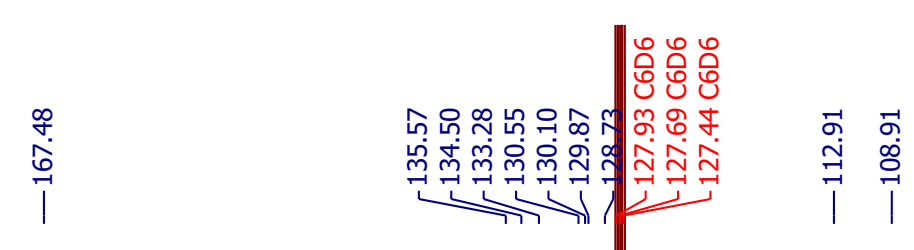

1i. 4-cyano-2,3,5,6-tetrafluoropyridine, $13 \mathrm{C}$ C6D6, $100 \mathrm{MHz}, 298 \mathrm{~K}$.

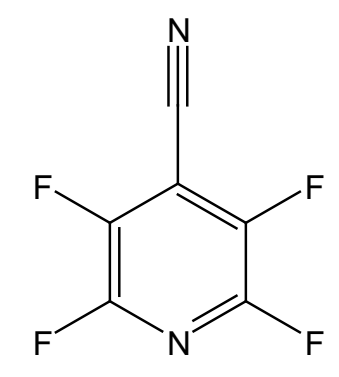


1i. 4-cyano-2,3,5,6-tetrafluoropyridine, 19F,

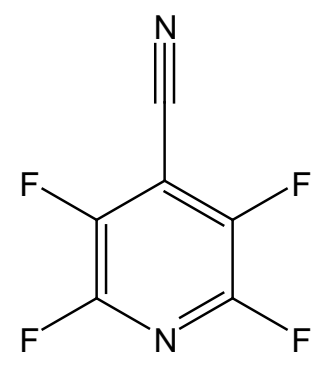

$F$

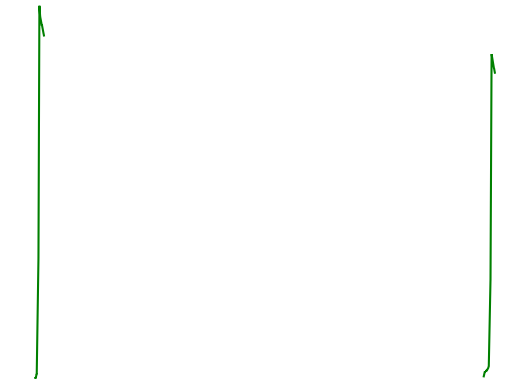

\begin{tabular}{|c|c|c|c|c|c|c|c|c|c|c|c|c|c|c|c|c|c|c|}
\hline & & & & & & & & & $\begin{array}{l}\stackrel{+}{\sim} \\
\stackrel{\sim}{N}\end{array}$ & & $\begin{array}{l}T^{\prime} \\
\stackrel{\sim}{\sim}\end{array}$ & & & & & & & \\
\hline 90 & 70 & 50 & 30 & 10 & -10 & -30 & -50 & -70 & $\begin{array}{c}-90 \quad-110 \\
\mathrm{f} 1(\mathrm{ppm})\end{array}$ & -130 & -150 & -170 & -190 & -210 & -230 & -250 & -270 & -290 \\
\hline
\end{tabular}




\section{1j. 4-benzoic-2,3,5,6-tetrafluorooctofluorotoluene, $1 \mathrm{H}$

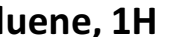

C6D6, $400 \mathrm{MHz}, 298 \mathrm{k}$.<smiles>O=C(Oc1c(F)c(F)c(C(F)(F)F)c(F)c1F)c1ccccc1</smiles>

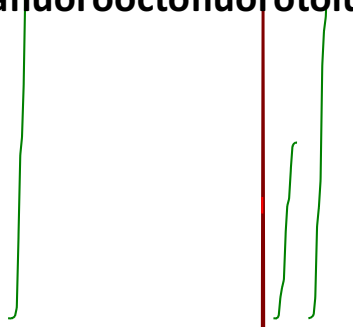


1j. 4-benzoic-2,3,5,6-tetrafluorooctofluorotoluene, $13 \mathrm{C}$ C6D6, $100 \mathrm{MHz}, 298 \mathrm{k}$.

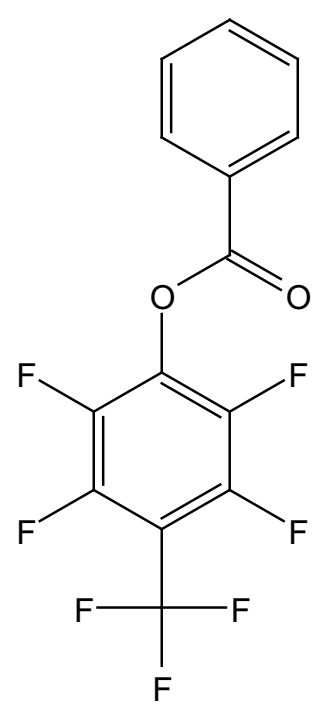

30




\section{1j. 4-benzoic-2,3,5,6-tetrafluorooctofluorotoluene, 19F} C6D6, 100 MHz, 298 k.
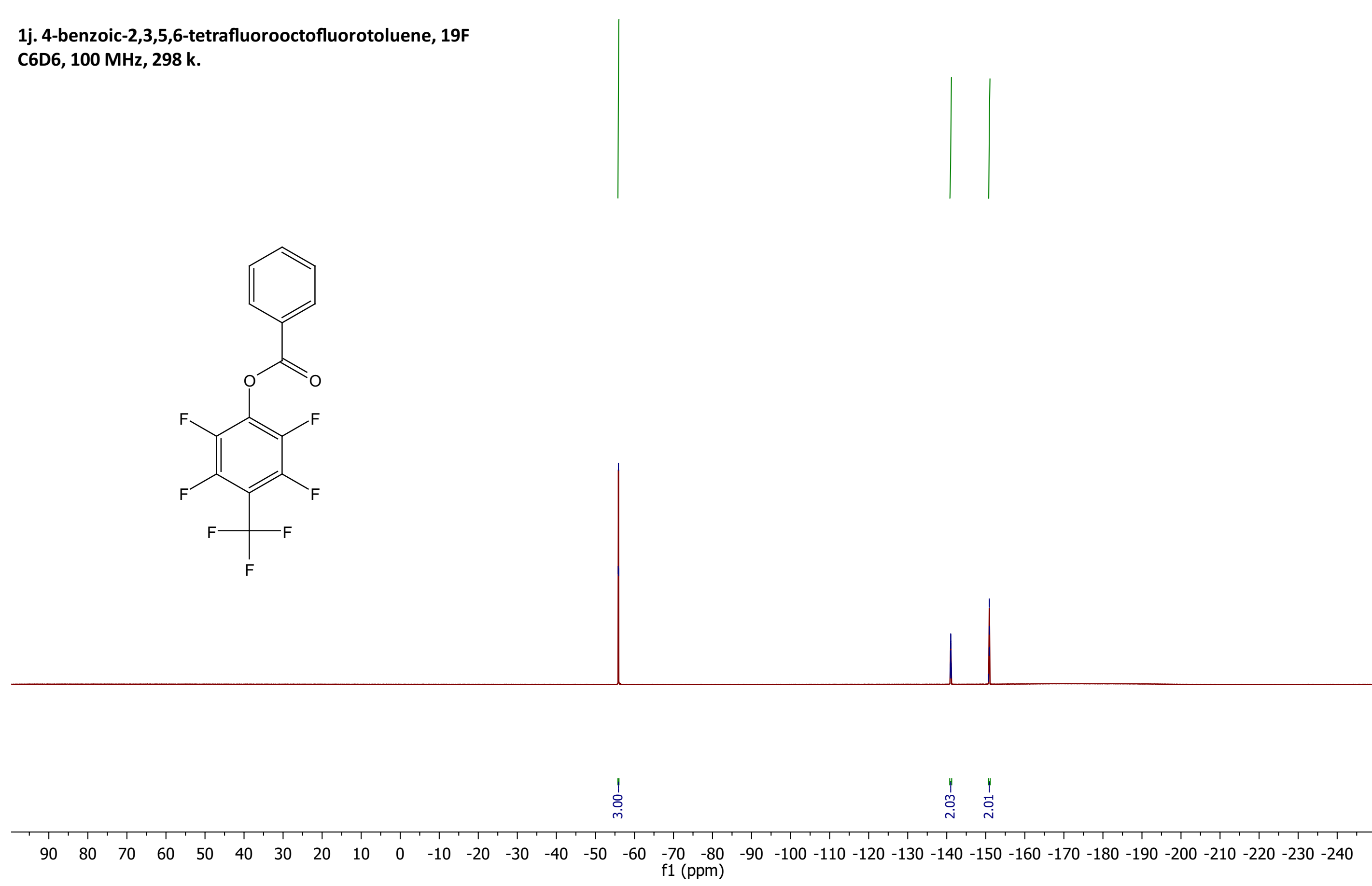
1k. 4-benzoic-2,3,5,6-tetrafluoroacetophenone, $1 \mathrm{H}$,

C6D6, $400 \mathrm{MHz}, 298 \mathrm{~K}$.
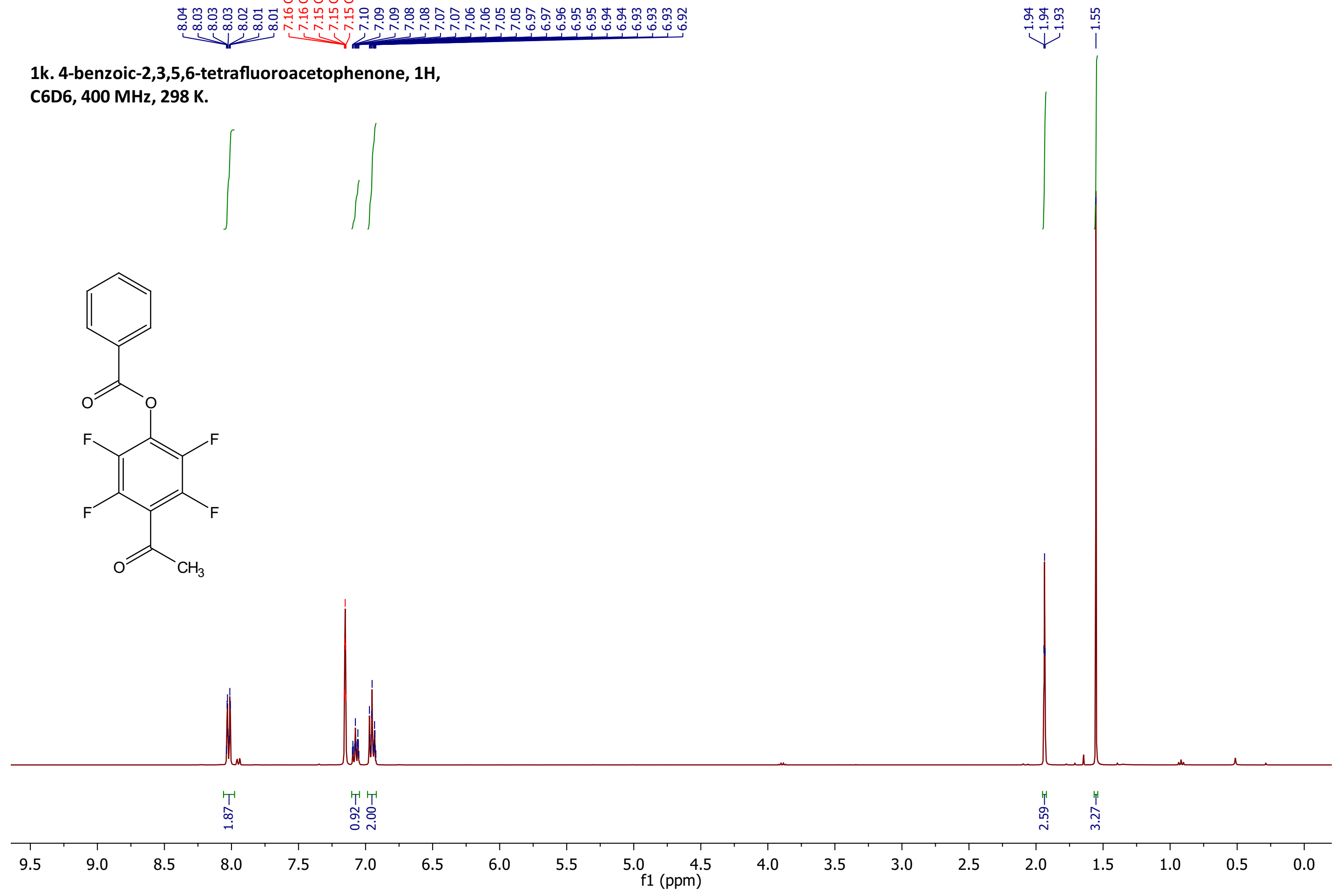
1k. 4-benzoic-2,3,5,6-tetrafluoroacetophenone, 13C, C6D6, $100 \mathrm{MHz}, 298 \mathrm{~K}$

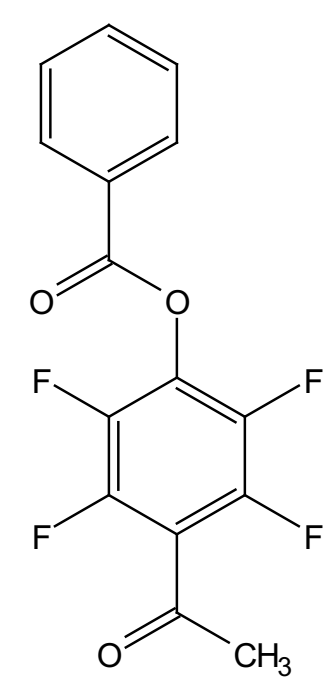

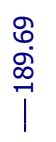

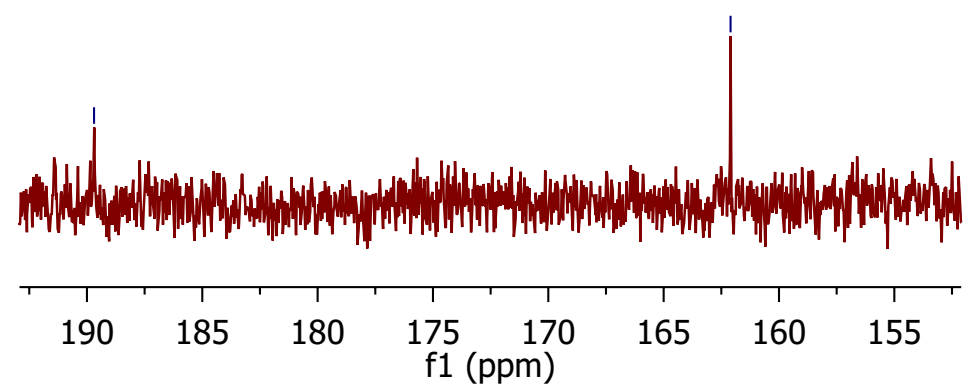


1k. 4-benzoic-2,3,5,6-tetrafluoroacetophenone, 19F,

C6D6, $400 \mathrm{MHz}, 298 \mathrm{~K}$.

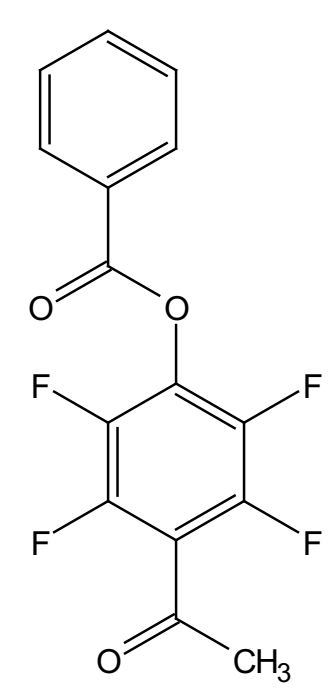

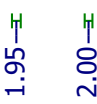

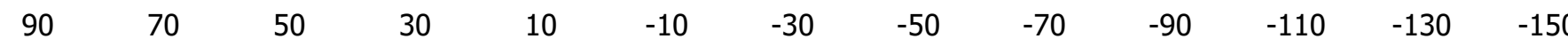
f1 (ppm) 


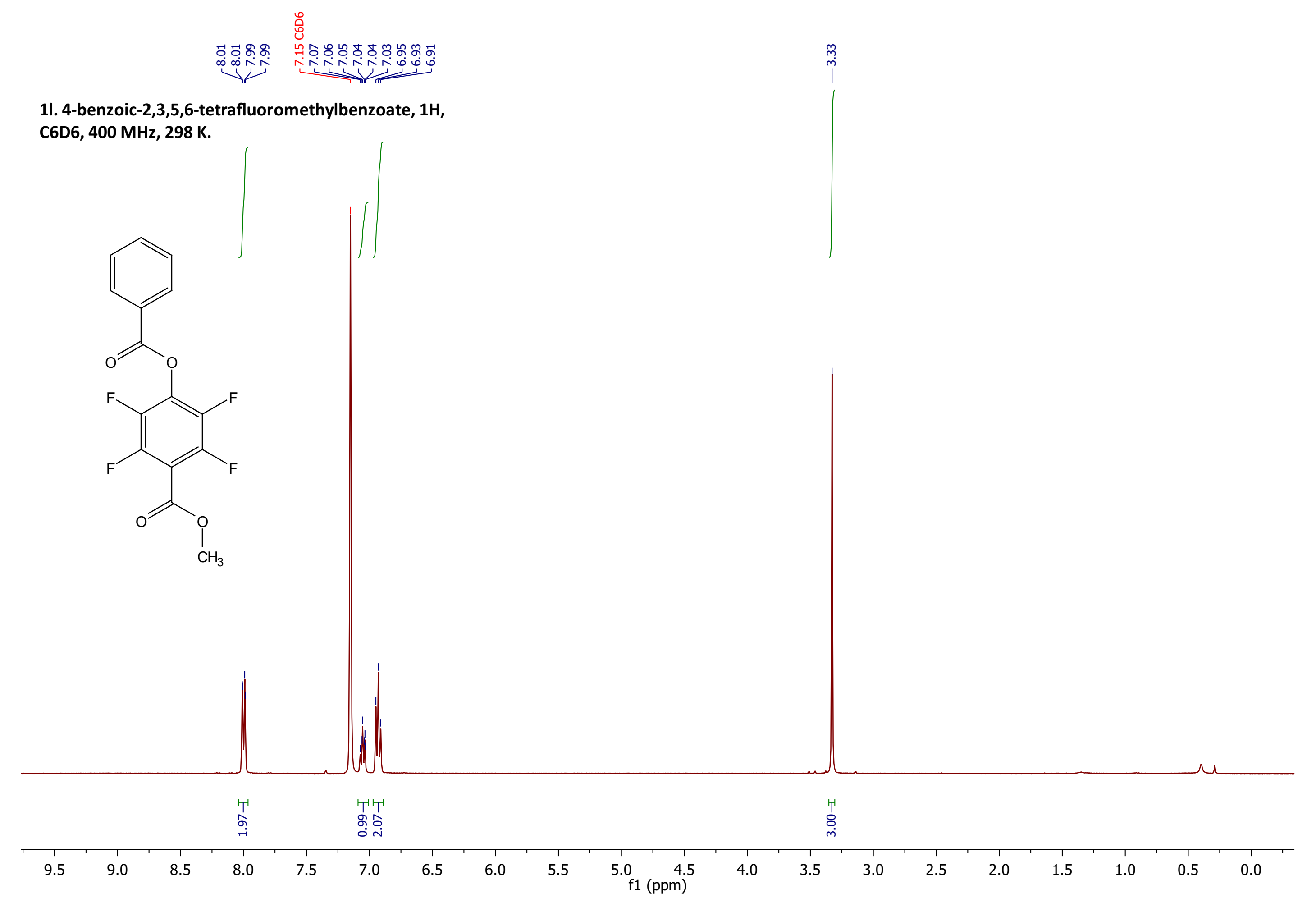




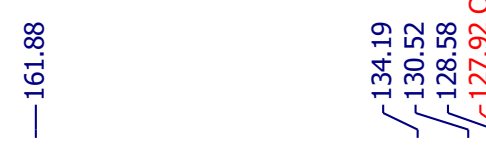

11. 4-benzoic-2,3,5,6-tetrafluoromethylbenzoate, 13C, C6D6, $100 \mathrm{MHz}, 298 \mathrm{~K}$.
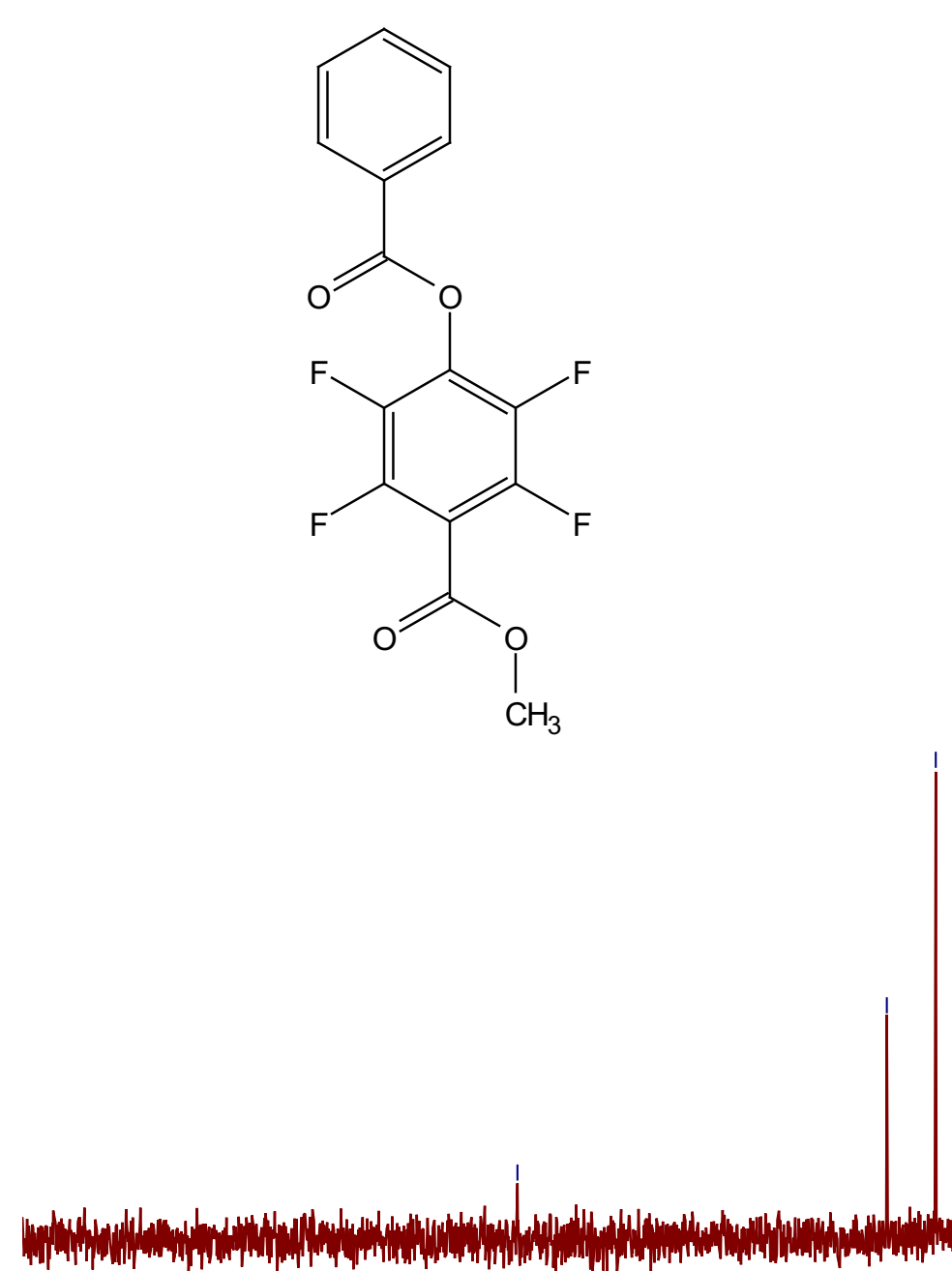

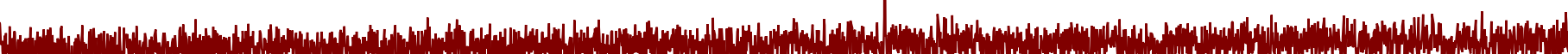

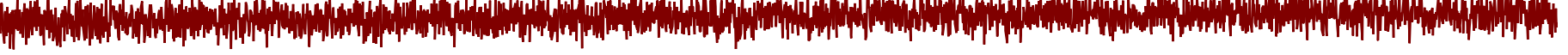


11. 4-benzoic-2,3,5,6-tetrafluoromethylbenzoate, 19F, C6D6, $100 \mathrm{MHz}, 298 \mathrm{~K}$.
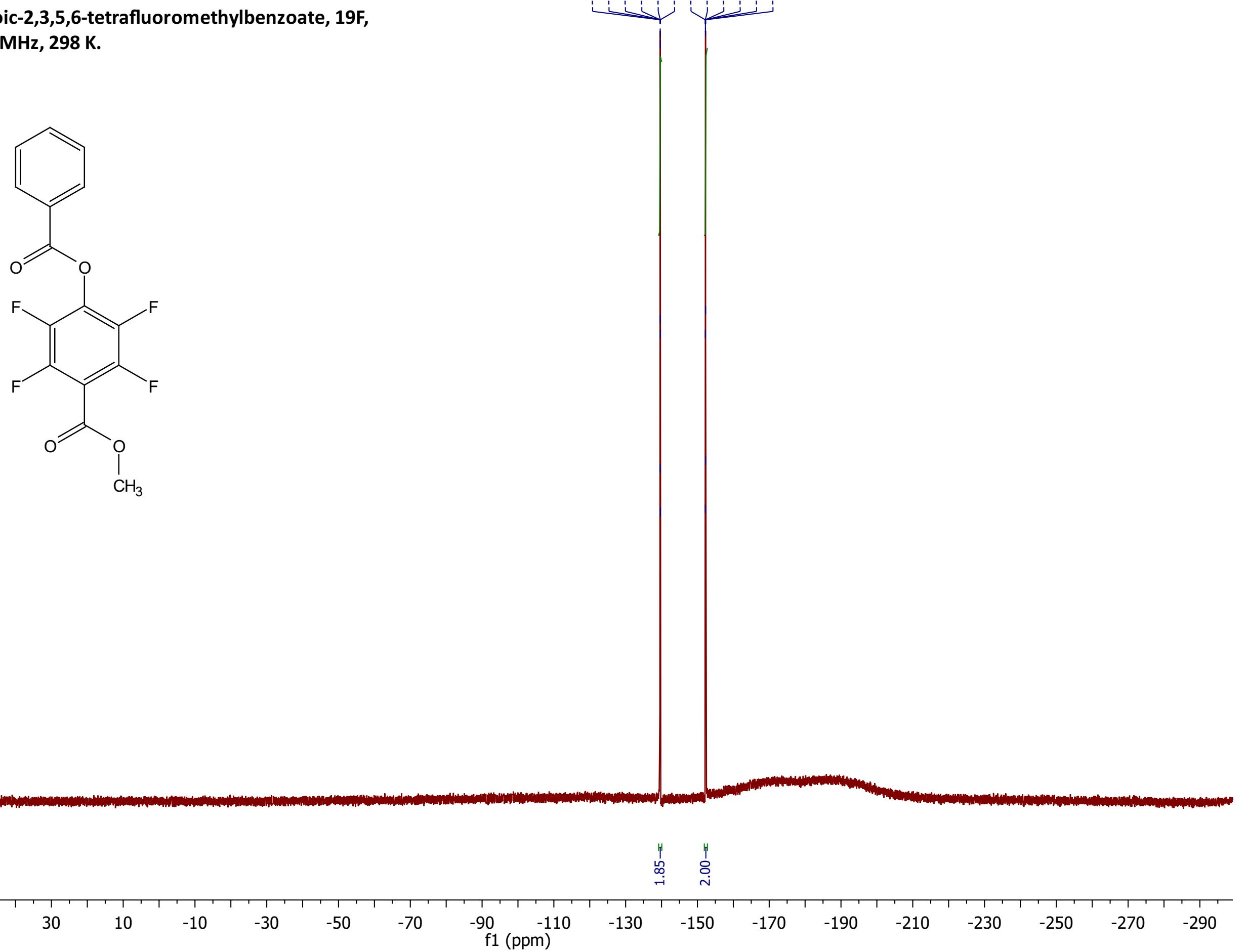

\begin{tabular}{|c|c|c|c|c|c|c|c|c|c|c|c|c|c|c|c|c|c|c|}
\hline & & & & & & & & & & & 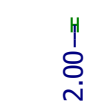 & & & & & & & \\
\hline 90 & 70 & 50 & 30 & 10 & -10 & -30 & -50 & -70 & $\begin{array}{ll}-90 & -110 \\
\mathrm{f} 1(\mathrm{ppm})\end{array}$ & -130 & -150 & -170 & -190 & -210 & $\begin{array}{l}1 \\
-230\end{array}$ & $\begin{array}{l}1 \\
-250\end{array}$ & $\begin{array}{l}1 \\
-270\end{array}$ & -290 \\
\hline
\end{tabular}


1m. phenyl-2,3,5,6-tetrafluorophenylsulfane,

$1 \mathrm{H}$,

C6D6, $400 \mathrm{MHz}, 298 \mathrm{~K}$.

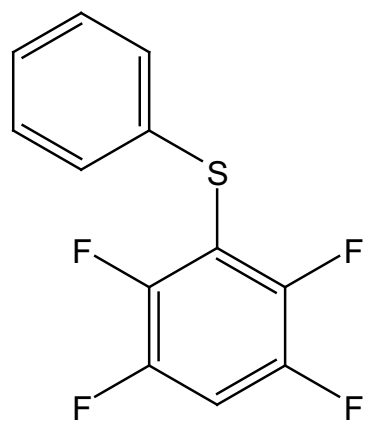

m
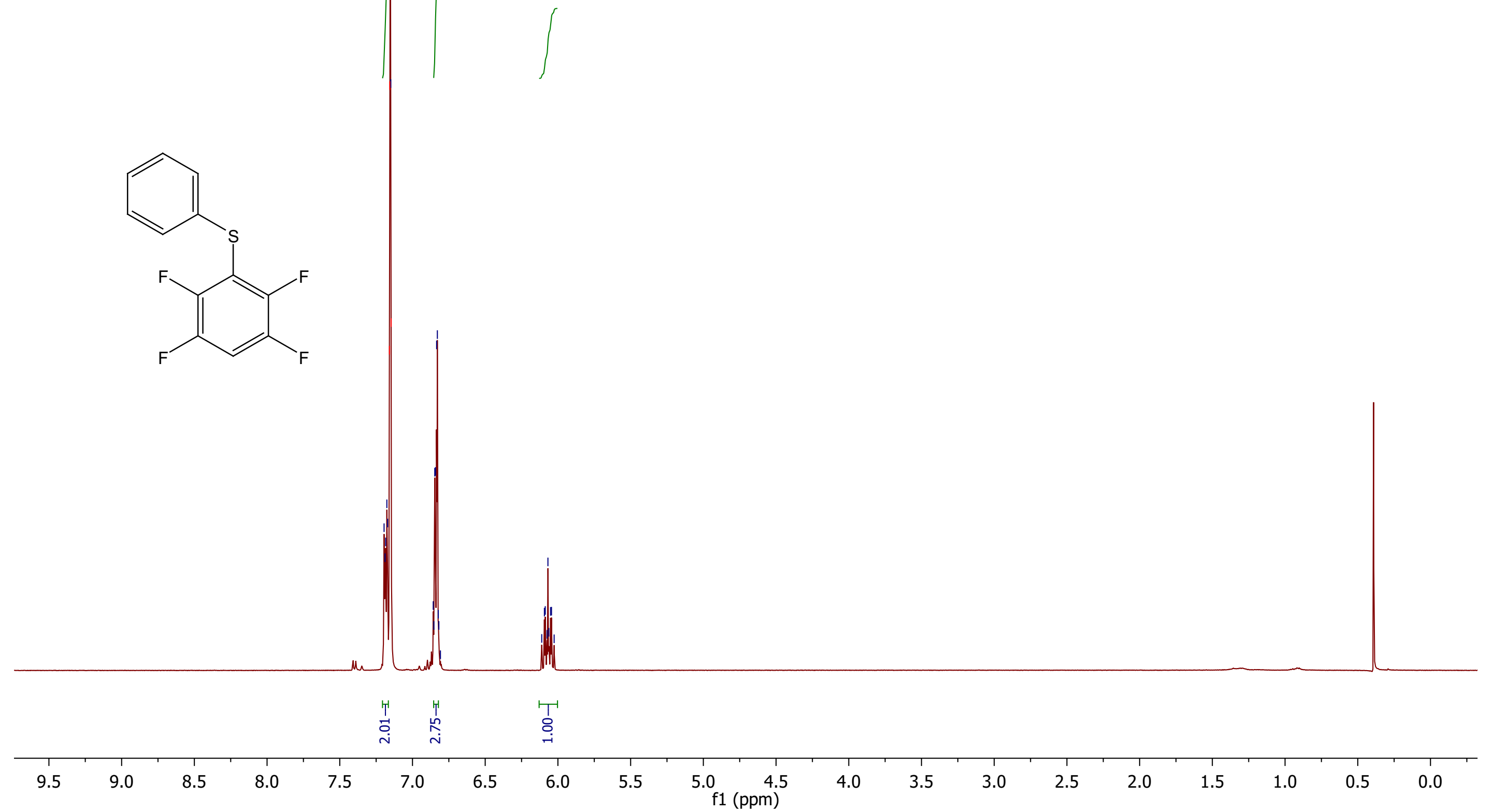
$1 \mathrm{~m}$. phenyl-2,3,5,6-tetrafluorophenylsulfane, $13 \mathrm{C}$, C6D6, $100 \mathrm{MHz}, 298 \mathrm{~K}$.

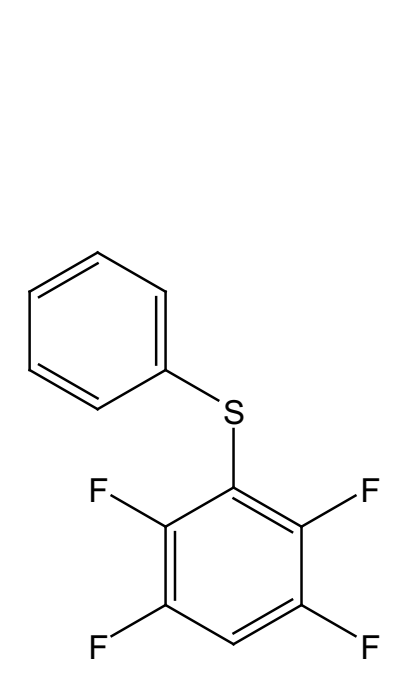

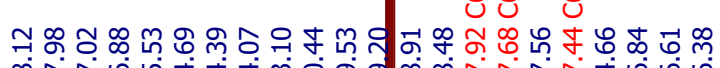

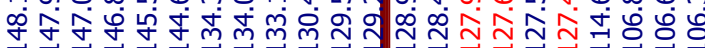

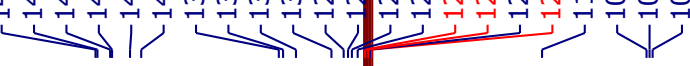
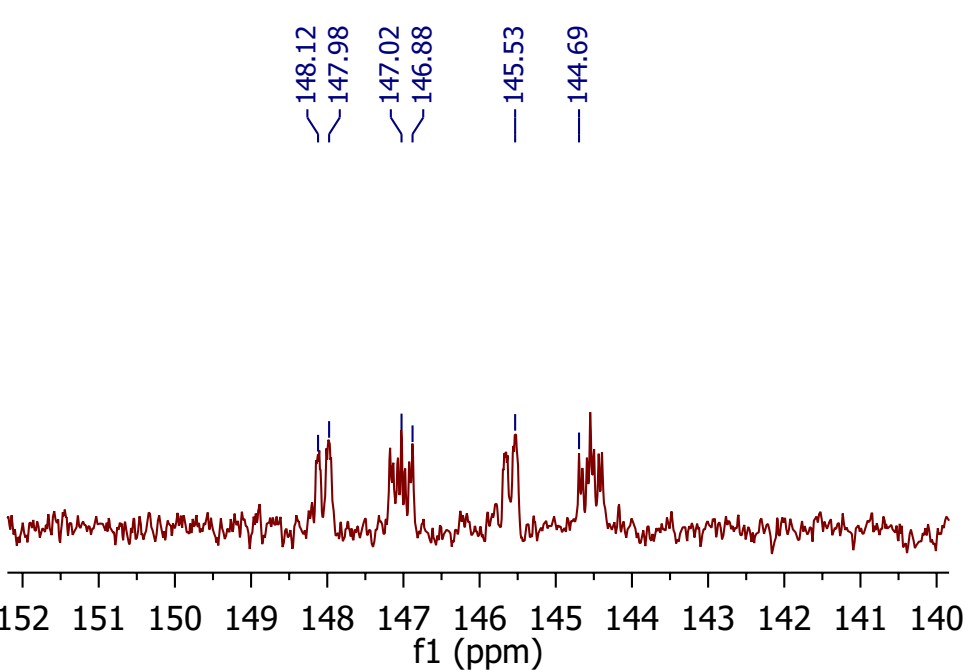

ilii

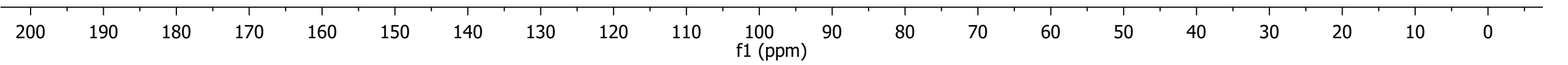


1m. phenyl-2,3,5,6-tetrafluorophenylsulfane, $19 \mathrm{~F}$, C6D6, $100 \mathrm{MHz}, 298 \mathrm{~K}$.
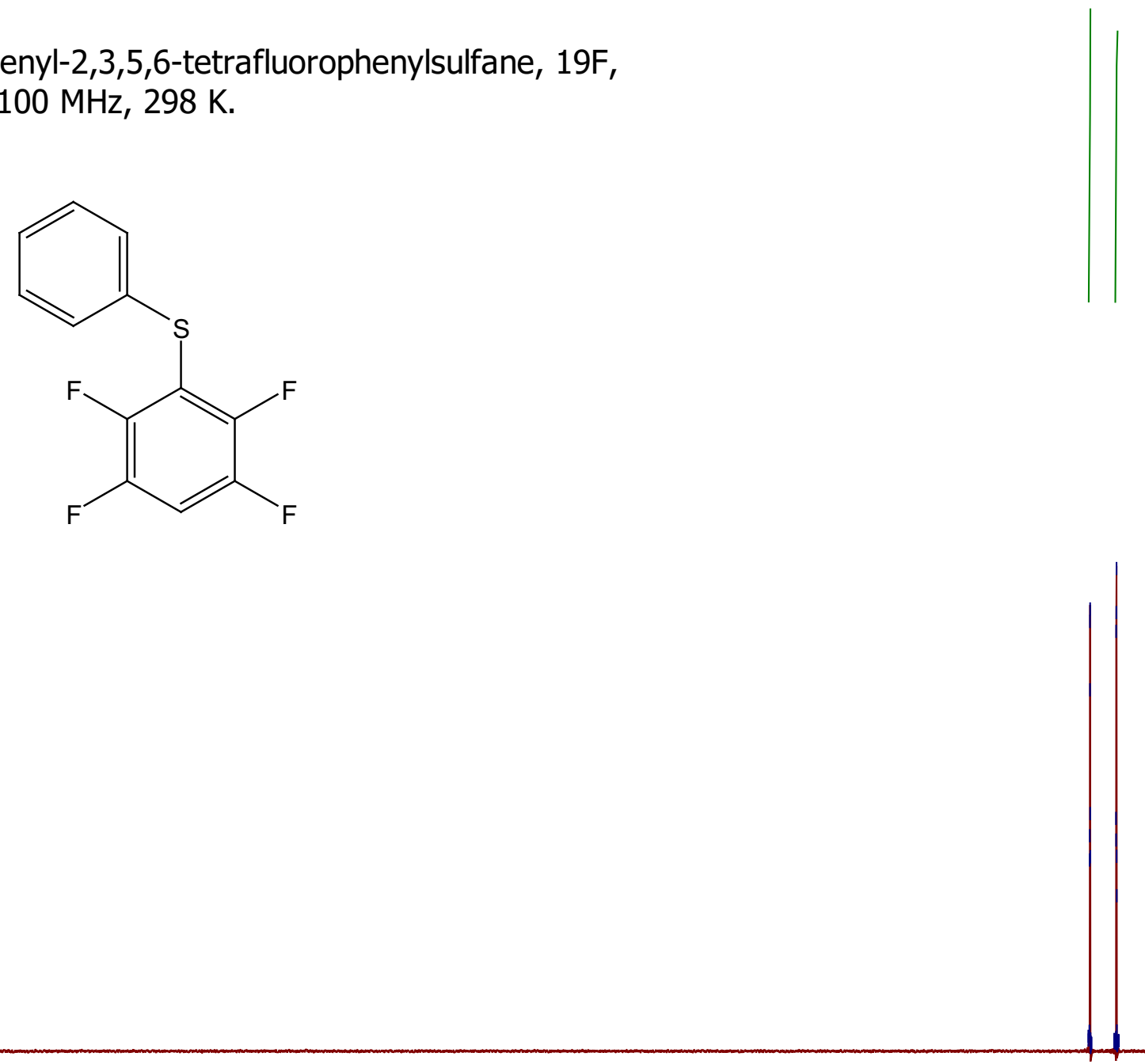

\begin{tabular}{|c|c|c|c|c|c|c|c|c|c|c|c|c|c|c|c|c|c|c|c|}
\hline & & & & & & & & & & & $\stackrel{!}{\stackrel{m}{n}}$ & & & & & & & & \\
\hline 90 & 70 & 50 & 30 & 10 & -10 & -30 & -50 & -70 & $\begin{array}{r}-90 \\
\text { f1 }\end{array}$ & $\frac{-110}{m)}$ & -130 & -150 & -170 & -190 & -210 & -230 & -250 & -270 & -290 \\
\hline
\end{tabular}


1n. Phenyl-2,3,5,-trifluorophenylsulfane, $1 \mathrm{H}$ C6D6, $400 \mathrm{MHz}, 298 \mathrm{~K}$.
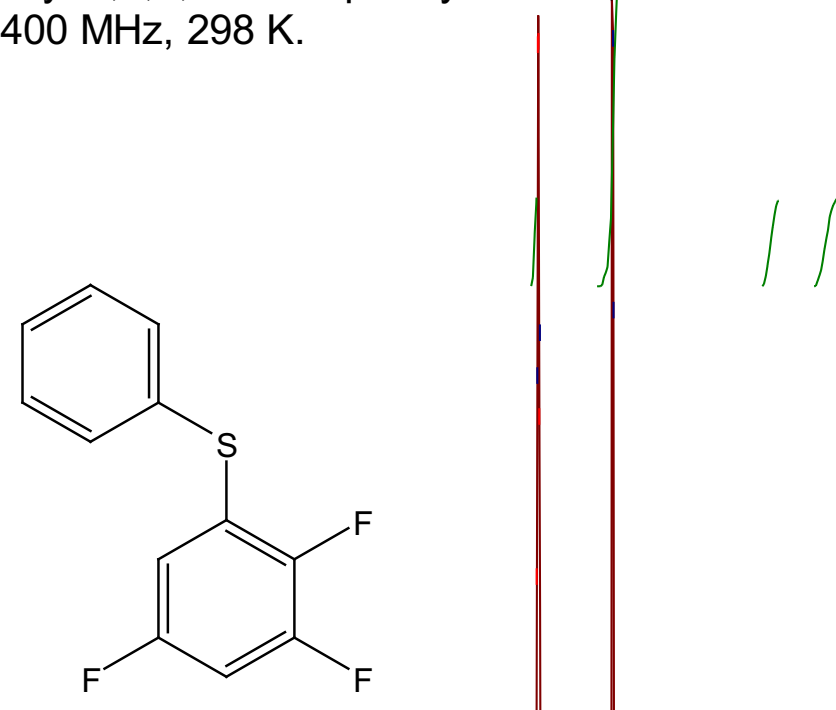

$$
\text { . }
$$

\begin{tabular}{|c|c|c|c|c|c|c|c|c|c|c|c|c|c|c|c|c|c|c|c|}
\hline & & & & & 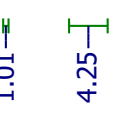 & 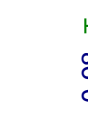 & $\begin{array}{l}1 \\
0 \\
0 \\
0 \\
0\end{array}$ & & & & & & & & & & & & \\
\hline T & $T$ & $T$ & $T$ & 1 & 1 & $T$ & 1 & $T$ & $T$ & $T$ & $T$ & $T$ & $T$ & $T$ & $T$ & $T$ & $T$ & $T$ & $T$ \\
\hline 9.5 & 9.0 & 8.5 & 8.0 & 7.5 & 7.0 & 6.5 & 6.0 & 5.5 & 5.0 & $\begin{array}{c}4.5 \\
\mathrm{f} 1(\mathrm{ppm})\end{array}$ & 4.0 & 3.5 & 3.0 & 2.5 & 2.0 & 1.5 & 1.0 & 0.5 & 0.0 \\
\hline
\end{tabular}

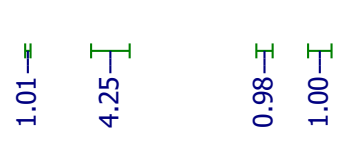

ᄋ̊ 
1n. Phenyl-2,3,5,-trifluorophenylsulfane, 13C C6D6, $100 \mathrm{MHz}, 298 \mathrm{~K}$.

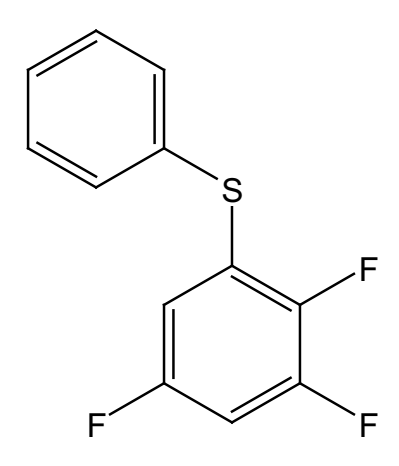

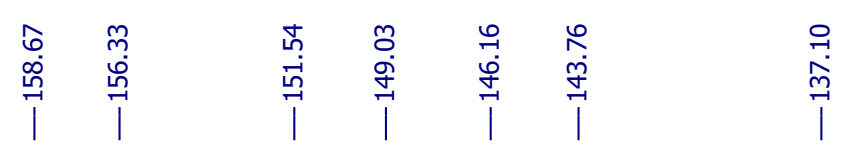
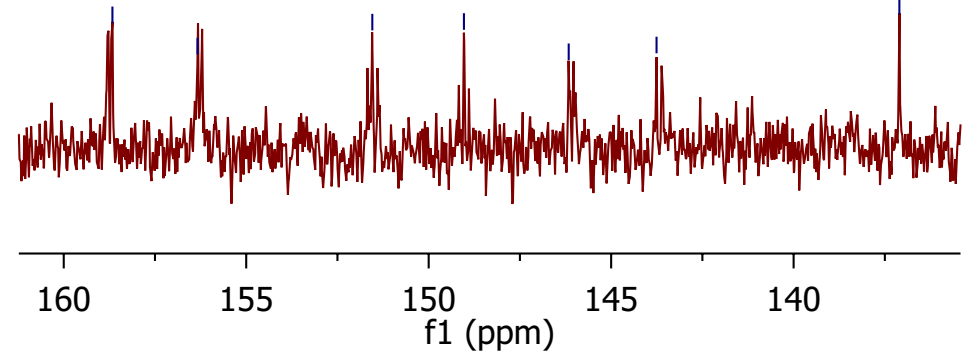

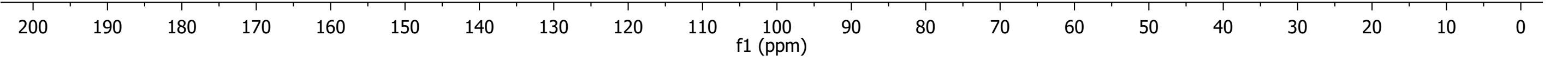


1n. Phenyl-2,3,5,-trifluorophenylsulfane, $19 \mathrm{~F}$

C6D6, $100 \mathrm{MHz}, 298 \mathrm{~K}$.
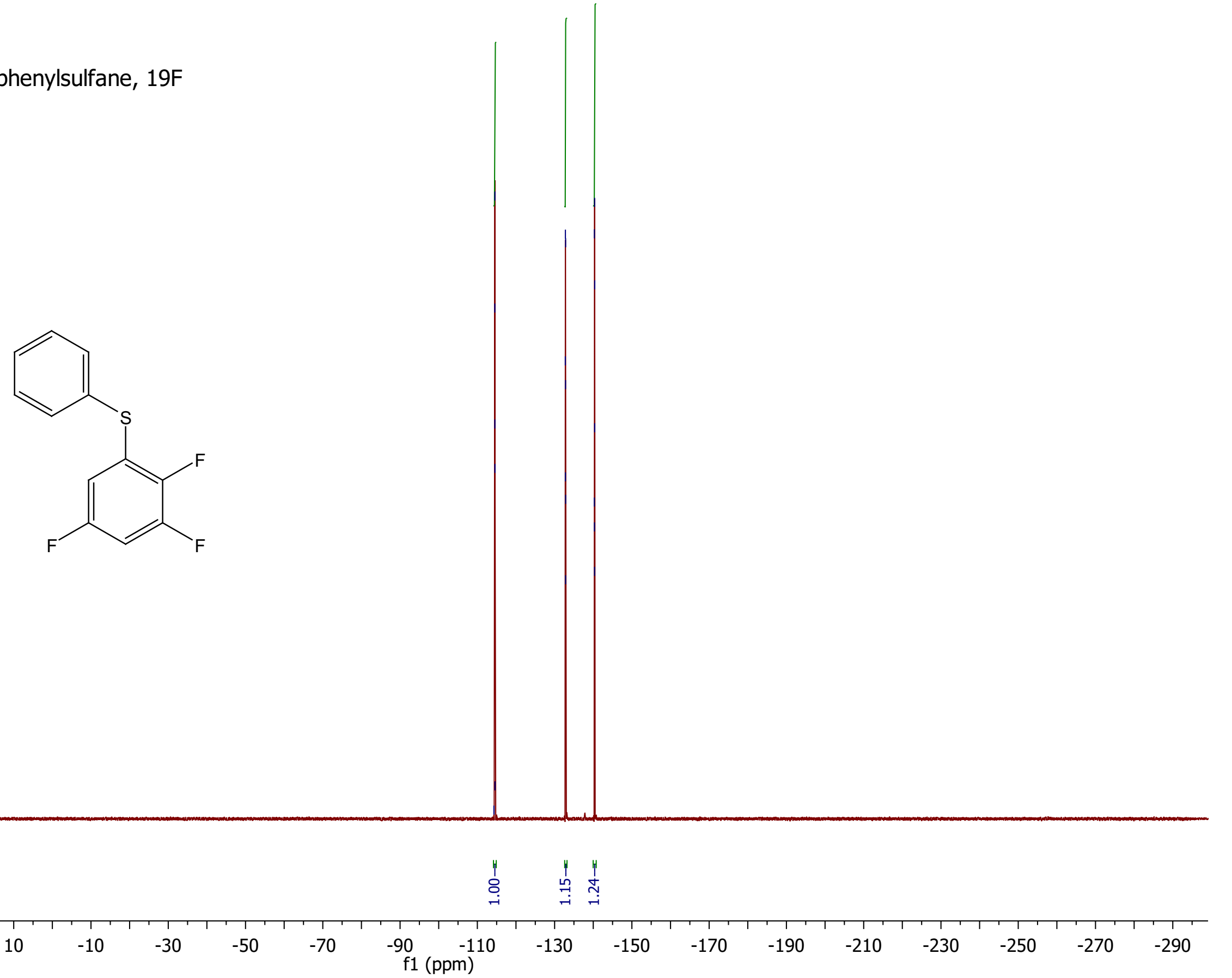


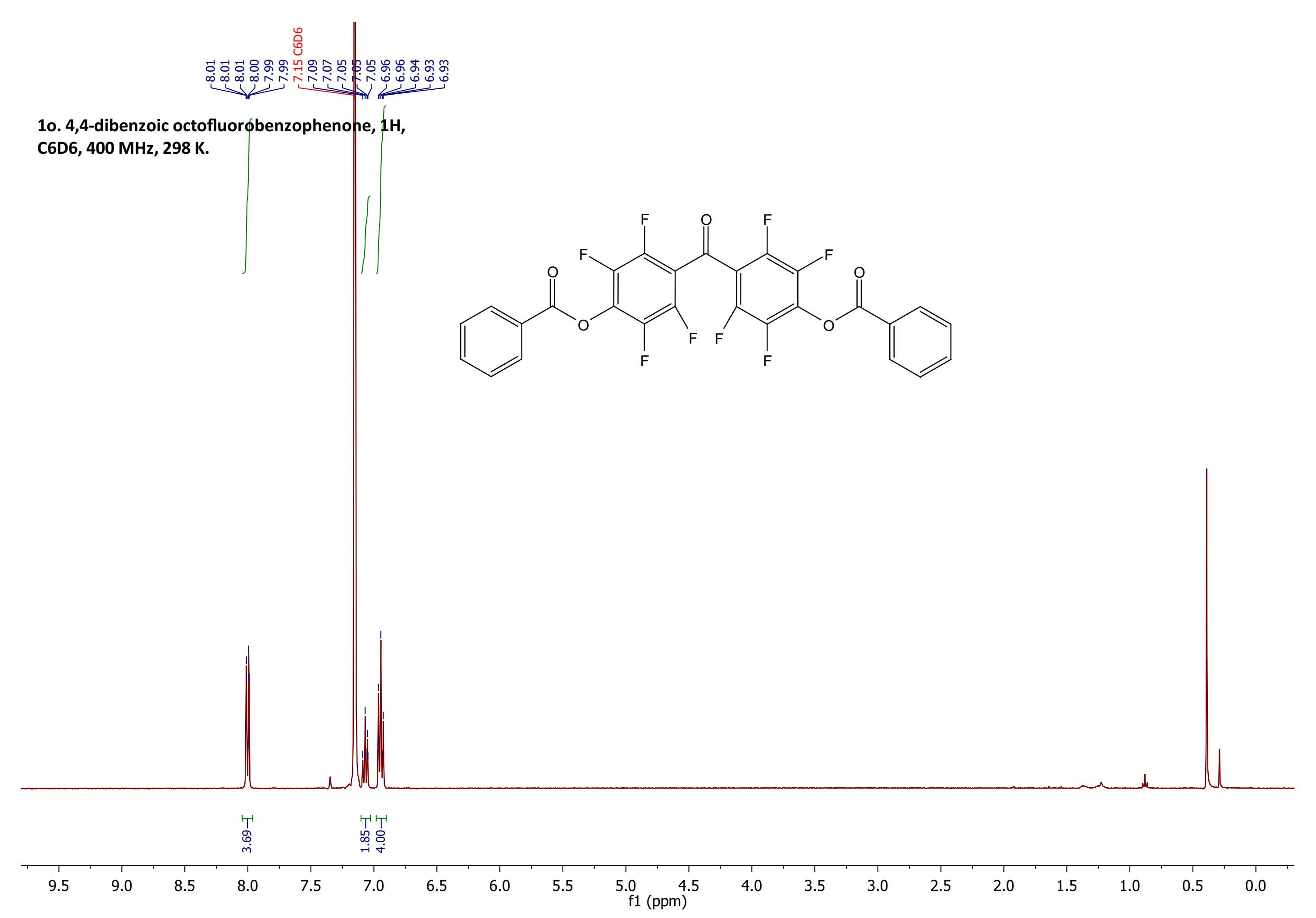


10. 4,4-dibenzoic octofluorobenzophenone, 13C, C6D6, $100 \mathrm{MHz}, 298 \mathrm{~K}$.

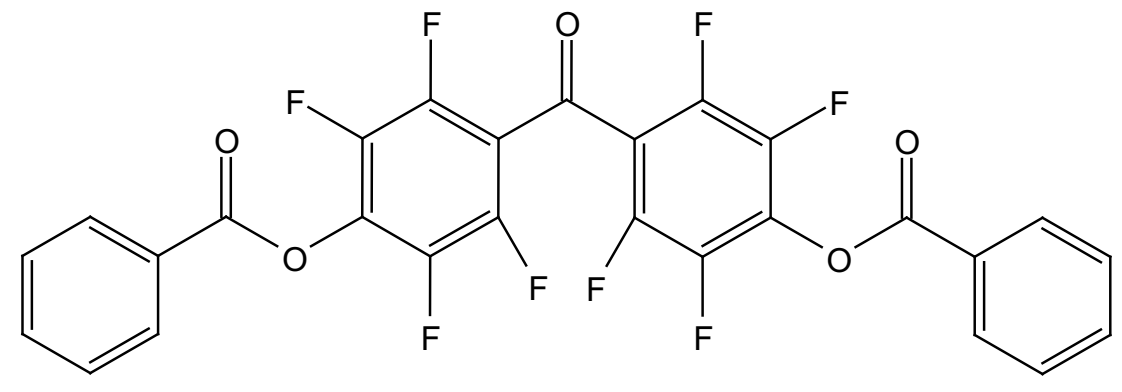

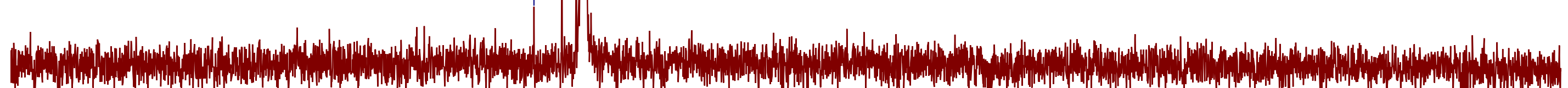

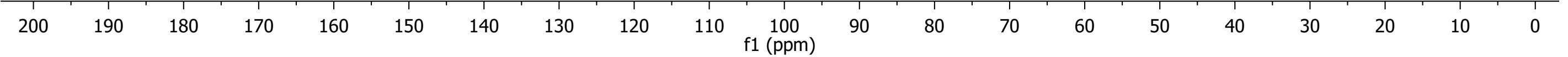


10. 4,4-dibenzoic octofluorobenzophenone, 19F,

C6D6, $100 \mathrm{MHz}, 298 \mathrm{~K}$.<smiles>O=C(Oc1c(F)c(F)c(C(=O)c2c(F)c(F)c(OC(=O)c3ccccc3)c(F)c2F)c(F)c1F)c1ccccc1</smiles>

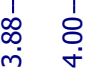

$-30$

$-50$

$\begin{array}{ccc}-70 & -90 \quad-110 \\ & \mathrm{f} 1(\mathrm{ppm})\end{array}$


1p. 4-benzoic-2,3,5,6-tetrafluoronitrobenzene, $1 \mathrm{H}$, C6D6, $400 \mathrm{MHz}, 298 \mathrm{~K}$

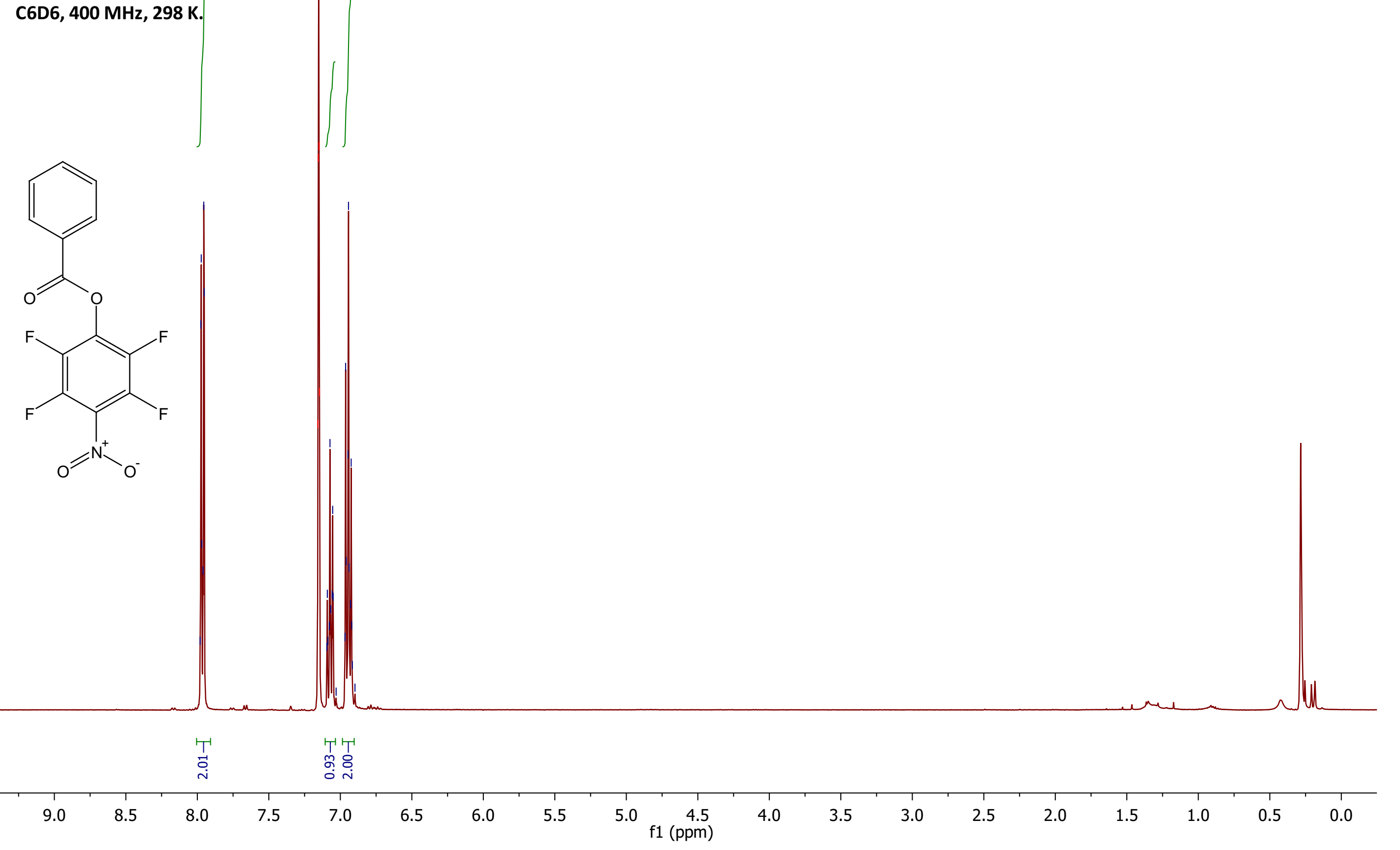


1p. 4-benzoic-2,3,5,6-tetrafluoronitrobenzene, 13C, C6D6, 100 MHz, 298 K.<smiles>O=C(Oc1c(F)c(F)c([N+](=O)[O-])c(F)c1F)c1ccccc1</smiles> 
1p. 4-benzoic-2,3,5,6-tetrafluoronitrobenzene, 19F,

C6D6, 400 MHz, $298 \mathrm{~K}$.

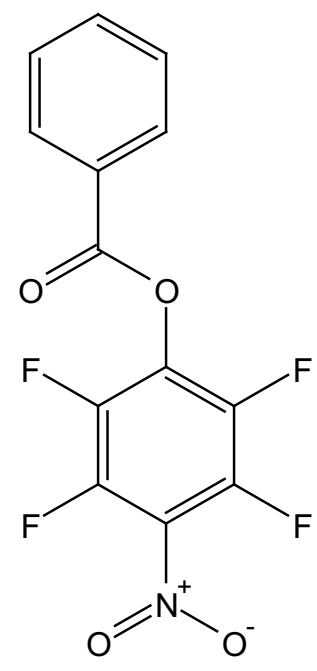

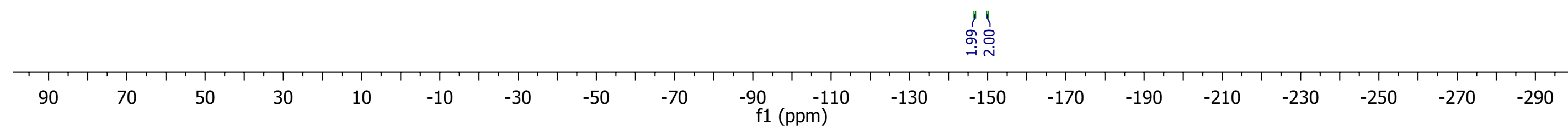


$1 p '$ '. 2,4-dibenzoic-3,5,6-trifluprobenzonitrile, $1 \mathrm{H}$,

C6D6, $400 \mathrm{MHz}, 298 \mathrm{~K}$.

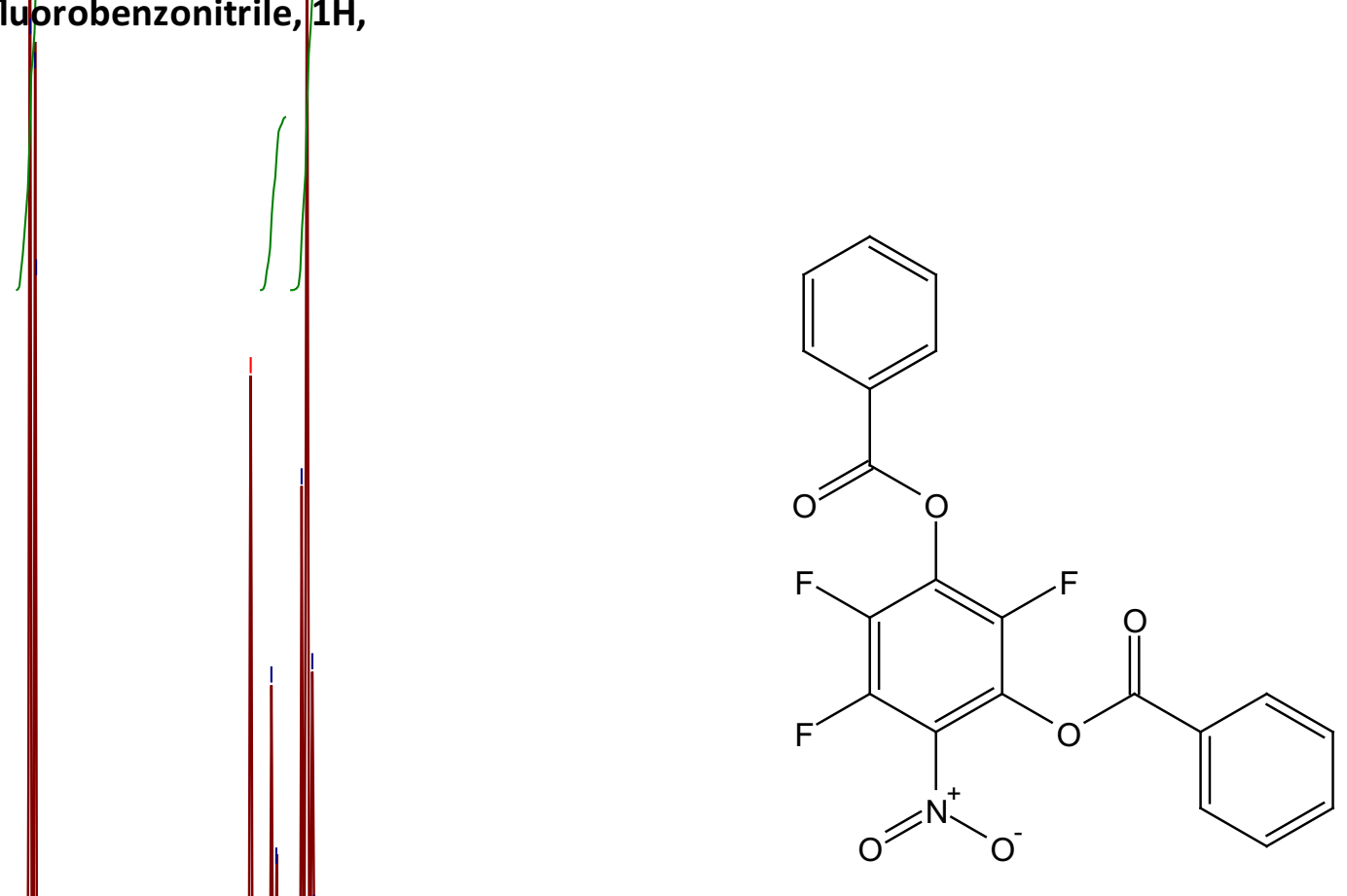

13

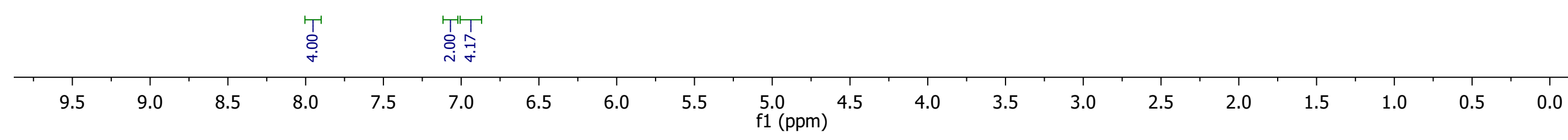




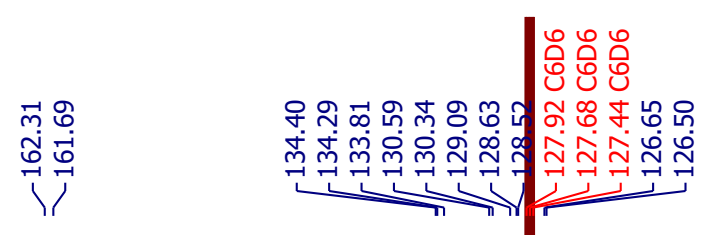

1p'. 2,4-dibenzoic-3,5,6-trifluorobenzonitrile, 13C, C6D6, $100 \mathrm{MHz}, 298 \mathrm{~K}$.

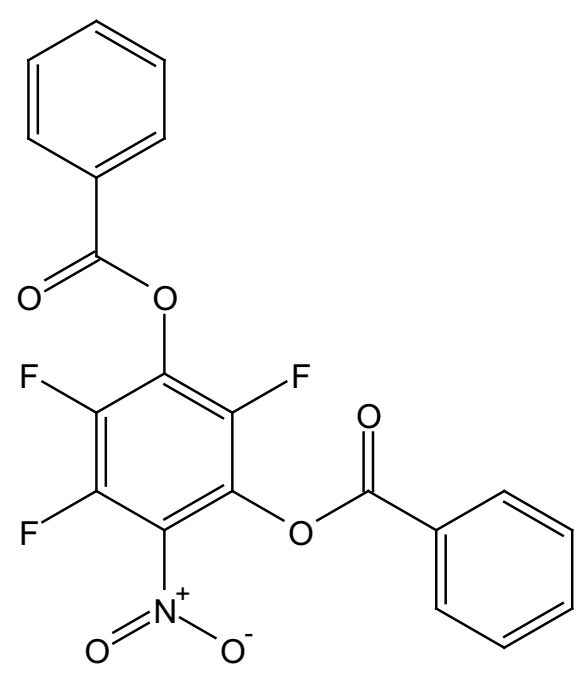

20


1p'. 2,4-dibenzoic-3,5,6-trifluorobenzonitrile, 19F, C6D6, $100 \mathrm{MHz}, 298 \mathrm{~K}$.

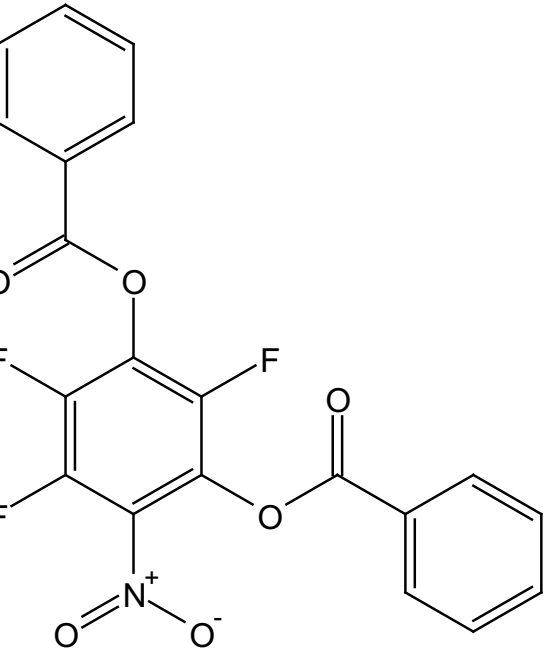

$\begin{array}{ll}-10 & -30\end{array}$




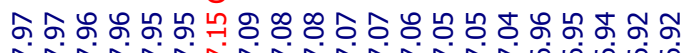

1q. 4-benzoic-2,3,5,6-tetrafluorobenzonitrile, $1 \mathrm{H}$,

C6D6 $400 \mathrm{MHz}, 298 \mathrm{~K}$.

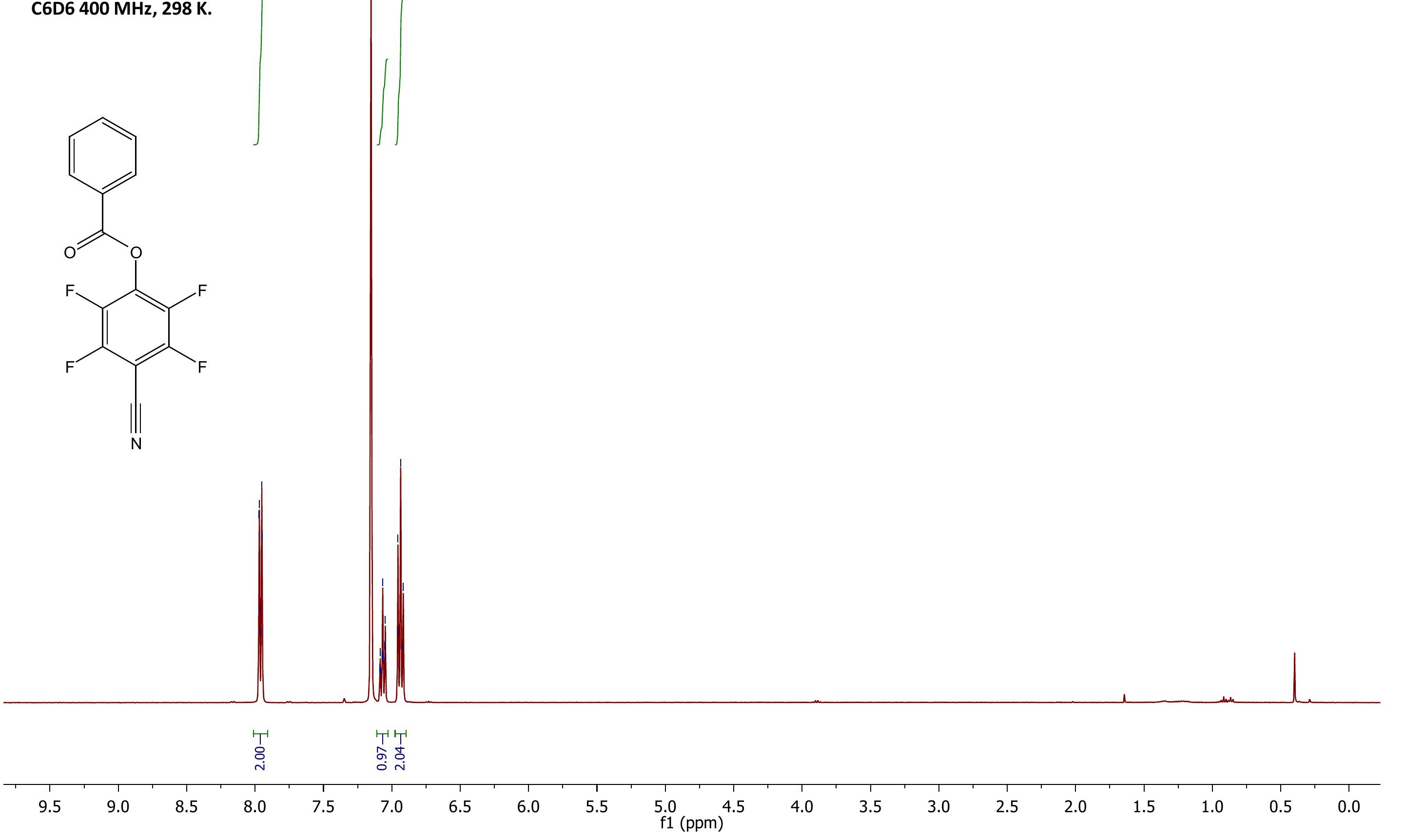




\begin{tabular}{|c|c|}
\hline 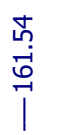 & 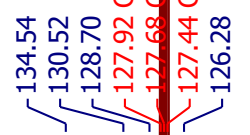 \\
\hline
\end{tabular}

1q. 4-benzoic-2,3,5,6-tetrafluorobenzonitrile, 13C, C6D6, 100 MHz, 298 K.

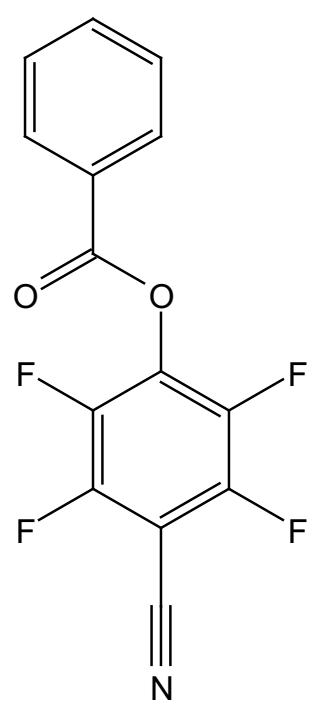


1q. 4-benzoic-2,3,5,6-tetrafluorobenzonitrile, 19F,

C6D6, $100 \mathrm{MHz}, 298 \mathrm{~K}$.

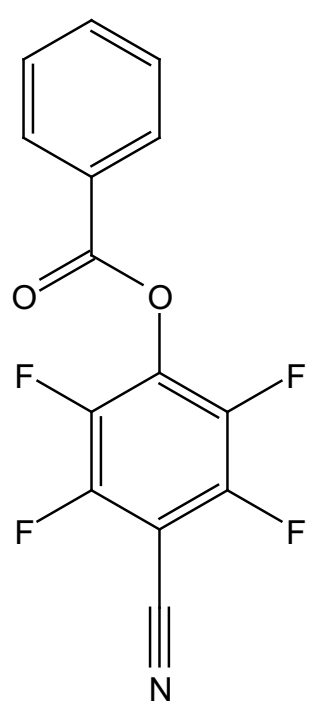

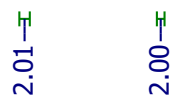

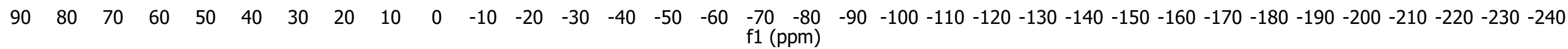


1q'. 2,4-dibenzoic-3,5,6-trifluorobenzonitrile, $1 \mathrm{H}$, C6D6, $400 \mathrm{MHz}, 298 \mathrm{~K}$.
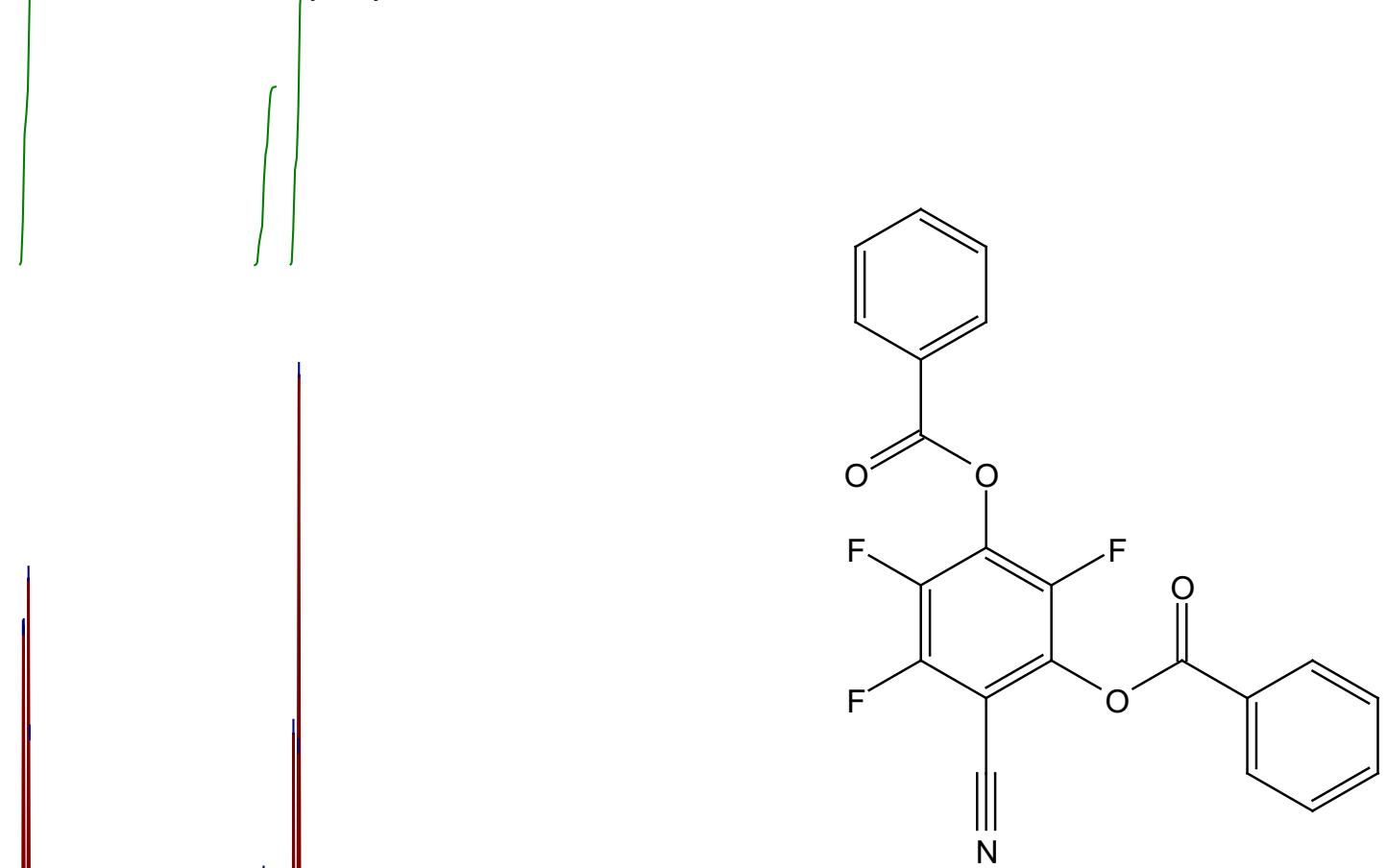

\begin{tabular}{|c|c|c|c|c|c|c|c|c|c|c|c|c|c|c|c|c|c|c|c|}
\hline 9.5 & 9.0 & 8.5 & 8.0 & 7.5 & 7.0 & 6.5 & 6.0 & 5.5 & $\begin{array}{l}5.0 \\
\text { f1 (ppm }\end{array}$ & 4.5 & 4.0 & 3.5 & 3.0 & 2.5 & 2.0 & 1.5 & 1.0 & 0.5 & 0.0 \\
\hline
\end{tabular}




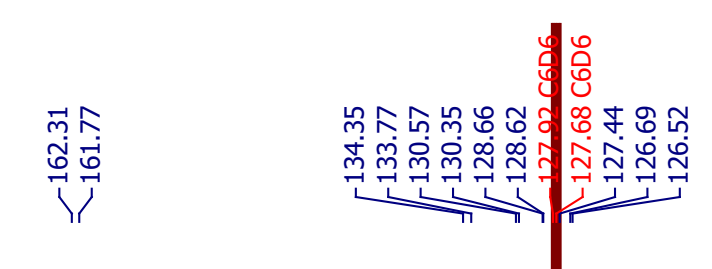

1q'. 2,4-dibenzoic-3,5,6-trifluorobenzonitrile, 13C, C6D6, $100 \mathrm{MHz}, 298 \mathrm{~K}$.

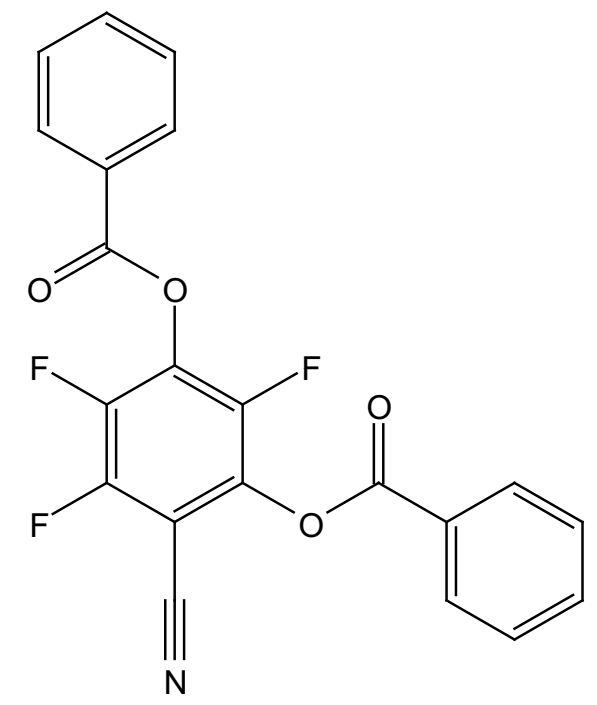

110

100
$\mathrm{f} 1(\mathrm{ppm})$

80

70
f1 (ppm) 
1q'. 2,4-dibenzoic-3,5,6-trifluorobenzonitrile, 19F, C6D6, $100 \mathrm{MHz}, 298 \mathrm{~K}$.

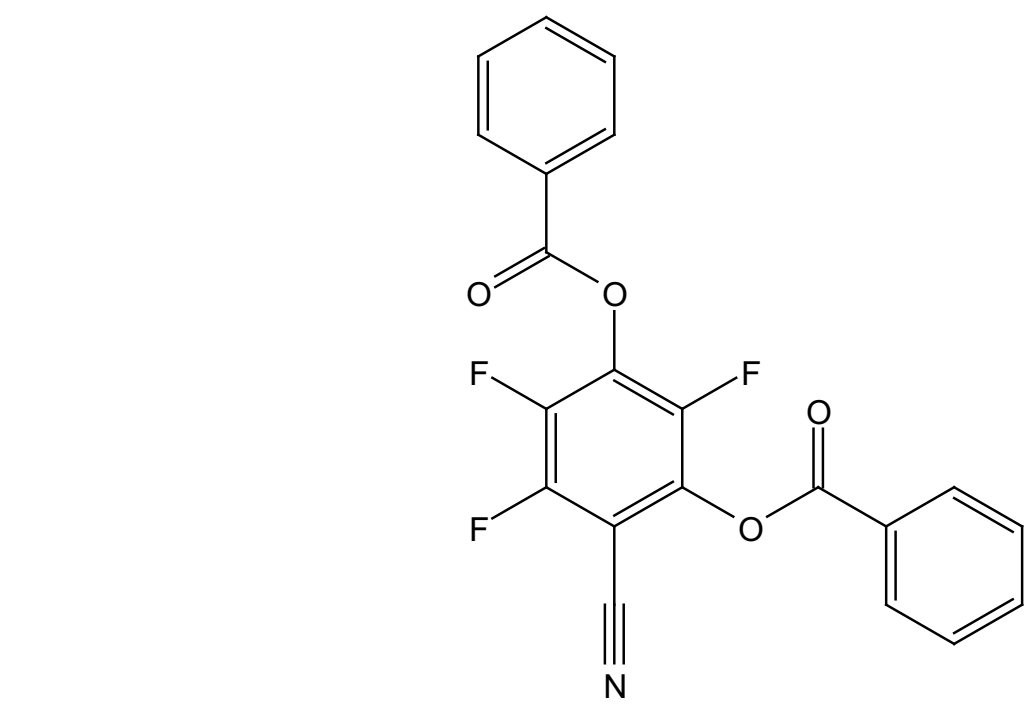

承

商

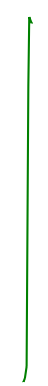


2a. Benzoyl fluoride, $1 \mathrm{H}$ C6D6, $400 \mathrm{MHz}, 298 \mathrm{~K}$.
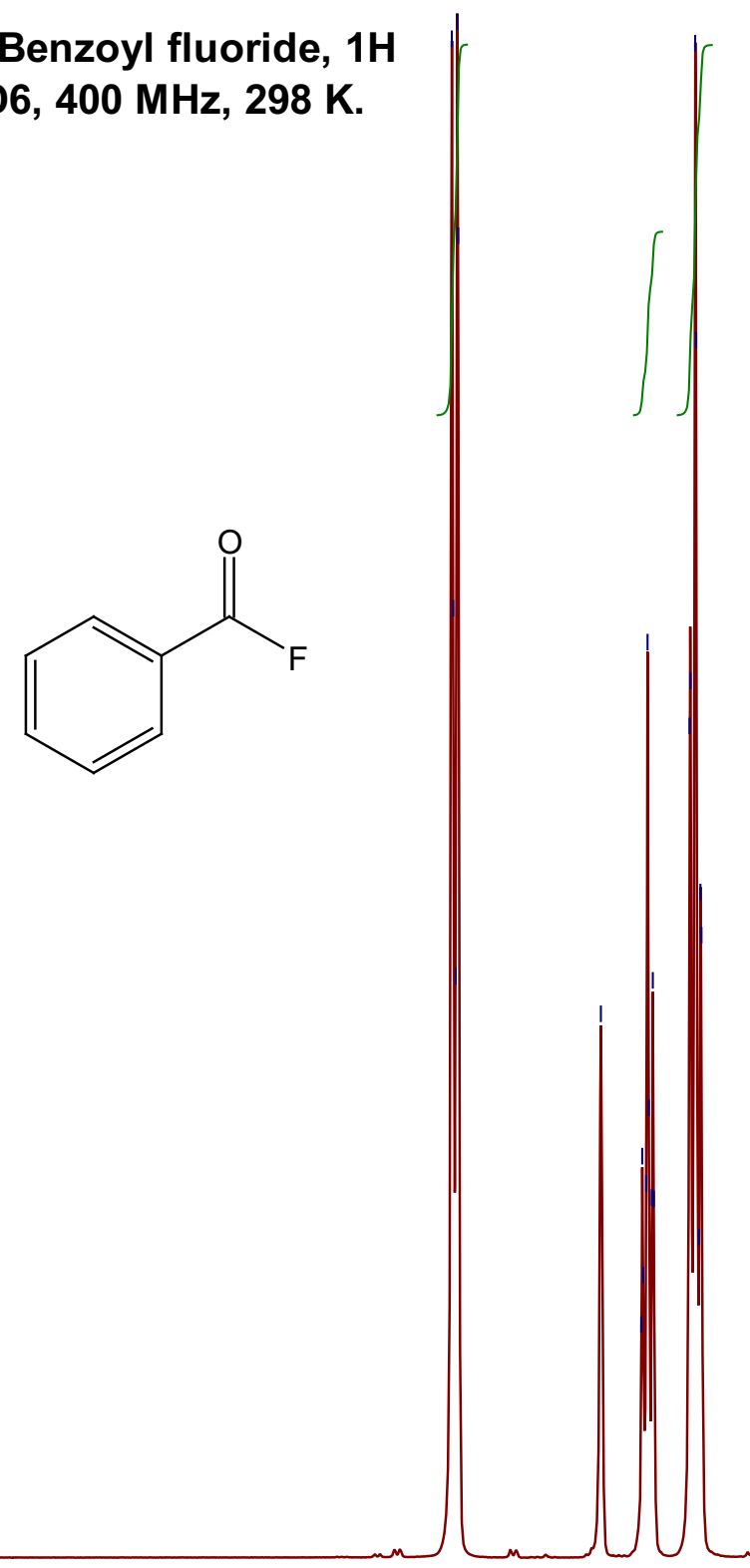

1

\begin{tabular}{|c|c|c|c|c|c|c|c|c|c|c|c|c|c|c|c|c|c|c|}
\hline & & & & & $\begin{array}{l}\text { 'Th } \\
\text { S' }\end{array}$ & & & & & & & & & & & & & \\
\hline 9.5 & 9.0 & 8.5 & 8.0 & 7.5 & 7.0 & 6.5 & 6.0 & 5.5 & $\begin{array}{c}5.0 \quad 4.5 \\
\text { f1 (ppm) }\end{array}$ & 4.0 & 3.5 & 3.0 & 2.5 & 2.0 & 1.5 & 1.0 & 0.5 & 0.0 \\
\hline
\end{tabular}


2a. Benzoyl fluoride, 136<smiles>O=C(F)c1ccccc1</smiles> 


\section{2a Benzoyl fluoride 19F} C6D6, $100 \mathrm{MHz}$, 298K

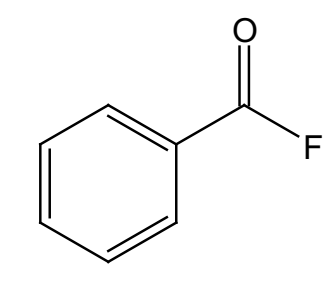

